\title{
Inflammatory bowel disease and oral health: systematic review and a meta-analysis
}

\begin{abstract}
Papageorgiou, Spyridon N ; Hagner, Martin ; Nogueira, Andressa Vilas Boas ; Franke, Andre ; Jäger,
\end{abstract} Andreas ; Deschner, James

\begin{abstract}
BACKGROUND: The objective of this systematic review was to systematically investigate whether there is an association between inflammatory bowel disease (IBD) and oral health. METHODS: Literature searches for randomized and non-randomized studies were performed up to January 2017. Risk of bias within studies was assessed with the Downs and Black checklist. Across-studies risk of bias was assessed with the GRADE framework. Quantitative synthesis was conducted with random-effects meta-analyses. RESULTS: A total of 9 cross-sectional studies including 1297 patients were included. IBD was associated with increased risk of periodontitis (332 more patients per 1000 patients; $95 \%$ confidence interval (CI): 257-388 patients; $\mathrm{p}<0.001)$ compared to non-IBD patients. Additionally, the Decayed-Missing-Filled-Teeth index of IBD patients was significantly worse than non-IBD patients (mean difference: $3.85 ; 95 \%$ CI: 2.36-5.34; $\mathrm{p}=0.005)$. Patients with ulcerative colitis had considerably worse oral health for most of the assessed factors, while the quality of overall evidence ranged from high to low, due to the observational nature of contributing studies. CONCLUSIONS: Inflammatory bowel disease was associated with significantly higher risk of periodontitis and worse oral health compared to non-IBD patients. However, longitudinal studies are needed in order to establish a causality link between IBD and periodontal disease.
\end{abstract}

DOI: https://doi.org/10.1111/jcpe.12698

Posted at the Zurich Open Repository and Archive, University of Zurich

ZORA URL: https://doi.org/10.5167/uzh-149324

Journal Article

Accepted Version

Originally published at:

Papageorgiou, Spyridon N; Hagner, Martin; Nogueira, Andressa Vilas Boas; Franke, Andre; Jäger, Andreas; Deschner, James (2017). Inflammatory bowel disease and oral health: systematic review and a meta-analysis. Journal of Clinical Periodontology, 44(4):382-393.

DOI: https://doi.org/10.1111/jcpe.12698 


\section{Title Page}

\section{Inflammatory bowel disease and oral health: systematic review and a meta-analysis}

Spyridon N. Papageorgiou ${ }^{1}$, Martin Hagner ${ }^{2}$, Andressa Vilas Boas Nogueira ${ }^{3}$, Andre Franke ${ }^{4}$, Andreas Jäger ${ }^{5}$, James Deschner ${ }^{6}$

${ }^{1}$ Clinic of Orthodontics and Pediatric Dentistry, Center of Dental Medicine, University of Zurich, Zurich, Switzerland

${ }^{2}$ Private Practice, Bonn, Germany

${ }^{3}$ Department of Diagnosis and Surgery, School of Dentistry, UNESP Univ Estadual Paulista, Araraquara, SP, Brazil

${ }^{4}$ Institute of Clinical Molecular Biology, Christian-Albrechts-University of Kiel, Kiel, Germany

${ }^{5}$ Department of Orthodontics, School of Dentistry, University of Bonn, Bonn, Germany

${ }^{6}$ Section of Experimental Dento-Maxillo-Facial Medicine, School of Dentistry, University of Bonn, Bonn, Germany.

Running title: Inflammatory bowel disease and periodontal disease

Keywords: inflammatory bowel disease; Crohn's disease; ulcerative colitis; periodontal disease; oral health; systematic review; meta-analysis

Correspondence address: Professor James Deschner, DMD, PhD, Section of Experimental Dento-MaxilloFacial Medicine, Center of Dento-Maxillo-Facial Medicine, University of Bonn, Welschonnenstr. 17, D-53111, Bonn; Tel: +49-(0)228-287-22650; Fax: +49-(0)228-287-22081; E-mail: james.deschner@ uni-bonn.de. 


\section{Conflict of interest and source of funding statement}

The authors declare no conflict of interest. No external funding was received for this study. SNP has received a scholarship from Forschungsgemeinschaft Dental e.V (FGD) for another project.

Acknowledgements: We thank Birte Groessner-Schreiber (University Hospital Schleswig-Holstein [UKSH], Kiel, Germany) for providing further information on their study and additional outcomes not reported in the paper. 


\section{Clinical Relevance}

Scientific rationale for study: A number of systematic diseases including cardiovascular disease, diabetes, metabolic syndrome and obesity have been associated with periodontal disease.

Principal findings: According to existing evidence from the literature, inflammatory bowel disease seems to be associated with increased risk of periodontal disease and increased caries experience, although this needs to be confirmed by further studies.

Practical implications: Evidence from this systematic review could guide future longitudingal studies with large sample sizes and more rigorous methodology, and help guide the clinical management of patients with inflammatory bowel disease. 


\section{Abstract}

Background: The objective of this systematic review was to systematically investigate whether there is an association between inflammatory bowel disease (IBD) and oral health.

Methods: Literature searches for randomized and non-randomized studies were performed up to January 2017. Risk of bias within studies was assessed with the Downs and Black checklist. Across-studies risk of bias was assessed with the GRADE framework. Quantitative synthesis was conducted with random-effects metaanalyses.

Results: A total of 9 cross-sectional studies including 1297 patients were included. IBD was associated with increased risk of periodontitis (332 more patients per 1000 patients; 95\% confidence interval: 257 to 388 patients; $\mathrm{p}<0.001)$ compared to non-IBD patients. Additionally, the Decayed-Missing-Filled-Teeth index of IBD patients was significantly worse than non-IBD patients (mean difference: $3.85 ; 95 \% \mathrm{CI}$ : 2.36 to $5.34 ; \mathrm{p}=0.005$ ). Patients with ulcerative colitis had considerably worse oral health for most of the assessed factors, while the quality of overall evidence ranged from high to low, due to observational nature of contributing studies.

Conclusions: IBD was associated with significantly higher risk of periodontitis and worse oral health compared to non-IBD patients. However, longitudinal studies are needed in order to establish a causality link between IBD and periodontal disease.

Registration: CRD42015019436 


\section{Introduction}

\section{Rationale}

Inflammatory bowel disease (IBD) and its two major disorders, Crohn's Disease (CD) and Ulcerative Colitis (CD), are a significant problem across the world, affecting about one in 200 people in developed countries and having a rising incidence and prevalence in developing countries (Molodecky et al. 2012). IBD is associated with many debilitating symptoms, including urgent diarrhoea, rectal bleeding, vomiting, anorexia, and lethargy, which frequently lead to poor psychosocial wellbeing with extensive consequences (Neovius et al. 2013). The financial burden of IBD for the healthcare system is considerable, with more than 2.2 billion US/year in the USA alone (Everhart \& Ruhl 2009). The development and progression of IBD is considered to be based on a complex combination of genetic influences and environmental factors (Ellinghaus et al. 2015), where disturbed host-microbiome interactions likely play a significant part (Jostins et al. 2012).

Several of these IBD-relevant factors and influences are also risk factors for periodontitis (Brandtzaeg 2001, Kinane \& Bartold 2007, Indriolo et al. 2011). In addition, studies have shown that the progression of disease in both IBD and periodontitis is characterized by immunoinflammatory processes and tissue destruction (Brandtzaeg 2001, Kinane \& Bartold 2007). Finally, several other oral manifestations of IBD have been reported in the last decades, including oral soft-tissue lesions, cobblestoning, aphthous ulcers, lymphadenopathy, and pyostomatitis (Dudeney 1969, Chan et al. 1991, Scheper \& Brand 2002, Harty et al. 2005).

To this end, periodontitis has been associated with a number of systemic diseases and conditions including diabetes, metabolic disorder, and obesity (Chaffee \& Weston 2010, Suvan et al. 2011, Nibali et al. 2013, Papageorgiou et al. 2015a). However, to our knowledge the possible association between IBD and periodontitis has not yet been adequately assessed in an evidence-based manner. Such evidence could identify similarities between the two entities that aid in the elucidation of the underlying biological principles of IBD and its forms, as well as provide information about an increased global burden of care for periodontal disease (Petersen \& Ogawa 2012) in IBD patients.

\section{Objectives}

The primary aim of this systematic review was to investigate whether the prevalence of periodontal disease is higher in patients with IBD compared to IBD-free patients, with the main research question being: "Do IBD patients have the same prevalence of periodontal disease as IBD-free patients?". The secondary aim was to assess whether IBD is associated with other parameters of oral health. 


\section{Methods}

\section{Protocol and Registration}

The protocol for this systematic review was made a priori, agreed upon from all authors and registered in PROSPERO (CRD42015019436). This systematic review is conducted according to the Cochrane Handbook (Higgins \& Green 2011) and reported according to the PRISMA statement (Liberati et al. 2009) and its extension for abstracts (Beller et al. 2013).

\section{Eligibility Criteria}

Inclusion and exclusion criteria were determined a priori (Appendix A). Both randomized controlled studies (RCTs) and non-RCTs that assessed any of the pre-specified periodontal or oral health outcomes in patients with and without IBD were included.

\section{Information Sources and Search}

Electronic general, open access, regional and grey literature databases were systematically searched up to January 2017 (Appendix B). MESH terms and relative keywords were used accordingly for each electronic database. No limitations were applied regarding publication year and status or language. The reference lists of included articles and relevant reviews were manually searched. Grey literature was searched through appropriate databases and registers. Authors were contacted when necessary for additional data or clarifications.

\section{Study Selection}

A study was judged as eligible, when none of the exclusion and all of the inclusion criteria were fulfilled. After removal of duplicates, articles were screened on the basis of title, abstract, and full-text. When the decision on the basis of title and abstract was inconclusive, the full-text article was acquired. Additional reports of the same trial/cohort were grouped together. When an identical study was published in more than one language, the most complete report was preferred, irrespective of language. Study selection was initially conducted by one author (SNP), who screened the titles and/or abstracts of retrieved studies. Subsequently, the full texts of potentially eligible studies (and from those abstracts which did not provide sufficient information to include/exclude) were screened by two authors independently (SNP and MH). Differences between the two authors were settled by a third author (JD). 


\section{Data Collection Process and Data Items}

Data extraction was performed independently by the same two review authors (SNP and MH) in a pre-defined and piloted collection form. Any disagreement was resolved by discussion with a third author (JD). Outcomes to be included were specified a priori at the protocol stage. All given post-treatment timepoints were to be included, but no such data existed.

\section{Risk of Bias in Individual Studies}

The risk of bias of RCTs was to be assessed with the Cochrane Collaboration's tool (Higgins \& Green 2011), but no RCTs were identified. The risk of bias of non-RCTs was assessed with a modified version of the Downs \& Black (1998) checklist.

\section{Summary Measures and Synthesis of Results}

Data were summarized and considered suitable for pooling, if similar disease groups were reported (or could be formed) and the same outcomes were reported. In cases of inadequate reporting, the missing data were calculated or requested from the authors. Data reported as medians and interquartile ranges were converted to means and standard deviations. If studies reported data for CD and UC, but not for IBD collectively, data were pooled prior to the meta-analysis (Higgins \& Green 2011).

Mean differences (MD) for continuous outcomes and Odds Ratios (ORs) for binary outcomes, together with their corresponding $95 \%$ confidence intervals (CI) were calculated. A random-effects model as proposed by DerSimonian \& Laird (1986) was chosen a priori as the primary method to estimate all pooled estimates, since clinical heterogeneity was expected (Papageorgiou 2014). The extent and impact of between-study heterogeneity was assessed by inspecting the forest plots for the localization of heterogeneity, by calculating the $\operatorname{tau}^{2}$ and the $\mathrm{I}^{2}$ statistic, respectively, and assessing the magnitude and direction of heterogeneity. The $95 \%$ CIs around $\mathrm{I}^{2}$ were calculated according to the non-central $\chi^{2}$ approximation of $\mathrm{Q}$. In case of considerable unexplained heterogeneity $\left(\mathrm{I}^{2}>75 \%\right)$ attempts were made to achieve homogeneity and, if they failed, metaanalysis was omitted. For meta-analyses with $\geq 3$ trials, 95\% prediction intervals (PrI) (Higgins et al. 2009) were calculated to predict effects in a future clinical setting. These incorporate observed heterogeneity in the meta-analysis estimates and provide a range of possible results that could exist in a future clinical setting. All analyses were performed in Stata version 12 (StataCorp LP, College Station, TX) with the macros metan, 
heterogi, and metareg. All $\mathrm{p}$ values were 2 -sided with $\alpha$ set at 5\%, except for the test of heterogeneity, where $\alpha$ was set at $10 \%$. No adjustment in the significance level was adopted to control for increased Type I error, since the nature of meta-analysis is observational per se and aims to identify existing significant associations.

\section{Risk of Bias Across Studies \& Additional Analyses}

The overall quality of evidence (confidence in effect estimates) for each of the main outcomes was rated using the GRADE approach (Guyatt et al. 2011). For this assessment, the risk of bias of each included trial was reassessed separately at outcome level. The minimal clinical important, large, and very large effects for continuous outcomes were conventionally defined (Sloan et al. 2006) as half, one, and two standard deviations, respectively (and as 1.5, 2.5, and 4.3 for the odds ratio). The standard deviation for an outcome was averaged from the existing trials. The produced forest plots were augmented with contours denoting the magnitude of the observed effects. Finally, the optimal information size (i.e. required meta-analysis sample size) was calculated for each outcome independently for $\alpha=5 \%$ and $\beta=20 \%$.

Random-effects meta-regressions were conducted for the comparison of IBD compared to healthy patients according to the following characteristics of IBD patients (a) mean age, (b) gender (assessed through the male/female ratio), (c) smoking (percentage of patients smoking), (d) IBD activity (percentage of patients with active disease), and (e) medication use, when at least 3 studies were included. Small study effects and signs of publication bias were planned to be assessed, if at least ten studies contributed to the meta-analyses. Sensitivity analyses were planned to be conducted to check the results' robustness according to follow-up, error of the method, definition of periodontitis, design of included studies (Papageorgiou et al. 2014a, Papageorgiou et al. 2015b), and improvement of the GRADE assessment.

\section{Results}

\section{Study Selection}

A total of 362 and 4 papers were identified through the electronic (Appendix C) and manual searches, respectively (Fig. 1). After removal of duplicates and initial screening, 43 papers were judged against the eligibility criteria, leaving a final number of 14 included papers (Fig. 1; Appendix C; Table 1). Six publications (Barro 2007, Barros et al. 2008, Brito et al. 2008, Silva 2008, Figueredo et al. 2011, Brito et al. 2013), referring to the same study and its follow-ups, were grouped, leaving a total of 9 unique studies that were finally included. 


\section{Study Characteristics}

The characteristics of the included studies are shown in Table 1. Eight case-control studies from different countries were included with a total of 744 IBD patients and 553 healthy individuals. Among the IBD patients described in the included studies 379 of them (56\%) had CD and $300(44 \%)$ had UC. In the studies that reported demographic characteristics, the male/female patient distribution was balanced in the IBD and healthy groups ( $45 \%$ to $51 \%$ in the IBD and $45 \%$ to $54 \%$ in the healthy group). Likewise, the mean age across studies was balanced between the IBD and healthy groups (37.2 and 35.7 years).

\section{Methodological Adequacy (Risk of Bias) of Individual Studies}

The risk of bias assessment for the included studies can be seen in Table 2. Serious methodological inadequacies that could be associated with bias were found in four studies for at least one methodological domain. Most problematic domains were the use of periodontal status as a criterion for patient enrollment in the study and incomplete reporting of patient characteristics or statistical analyses.

\section{Results of Individual Studies and Data Synthesis}

The results of the individual studies that were not included in the meta-analyses can be found in Appendix D. The performed meta-analysis comparing IBD patients with healthy patients overall, CD patients with healthy patients, and UC patients with healthy patients are shown in Tables 3-4, Fig. 2, and Appendix E-H. Compared to healthy patients, patients with IBD were significantly more likely to have periodontitis (Fig. 2), had significantly higher $\%$ of sites with large clinical attachement loss (3 to $5 \mathrm{~mm}$ ) higher mean pocket probing depth, higher periodontal treatment need, higher DMFT index, and more oral lesions. Patients with CD, likewise, were significantly more likely to have periodontitis, had significantly higher DMFT index, and significantly higher percentage of sites with pocket probing depth $>3 \mathrm{~mm}$ (Appendix G). Finally, patients with UC were significantly more likely to have periodontitis or oral lesions, had significantly fewer teeth (Appendix E), greater DMFT index (Appendix F), greater percentage of sites with clinical attachment loss $>3 \mathrm{~mm}$, and greater pocket probing depth (Appendix H).

\section{Risk of Bias Across Studies}


The risk of bias across studies (quality of evidence) according to the GRADE approach is summarized in Table 4 and Appendix I.

As far as the primary outcome of this review, prevalence of periodontitis, is concerned, high quality evidence supported the association of IBD generally (or CD and UC separately) with increased risk of periodontitis. IBD was associated with a higher risk of periodontitis by 332 patients per 1000 compared to healthy patients. The numbers-needed-to-treat indicated that for every 3 or 4 patients screened, an additional patient with periodontitis would be identified, if these patients had IBD. The subtype of IBD (CD or UC) had also a distinct effect on oral health, with UC patients being more severely affected compared to CD patients (additional increase in risk by 42 per 1000 patients).

As far as the two secondary outcomes of number of teeth and DMFT index of patient were concerned, low quality evidence indicated that IBD was associated with fewer teeth per patient and higher DMFT index. The only reason for the low GRADE score was the fact that it was based on observational non-experimenal studies that provided only a snapshot picture of the disease. Patients with IBD (either CD or UC) had an average of one tooth less $(\mathrm{p}=0.090)$ and a greater DMFT index by $3.85(\mathrm{p}=0.005)$. Likewise, to the prevalence of periodontitis, both the number of teeth and the DMFT index were more severely affected in UC patients than in CD patients compared to healthy patients (Appendix $\mathrm{G}$ and $\mathrm{H}$ ). This was also seen in an explorative post-hoc comparison between UC and CD patients, where UC patients had significantly fewer teeth, higher plaque index and greater attachment loss (Appendix $\mathbf{J})$.

\section{Additional Analyses}

Random-effect meta-regressions according to patient characteristics failed to identify any significant modifying effect on periodontitis, number of teeth, and DMFT index (Appendix K). Reporting biases were planned in the initial protocol, but could not be performed due to the limited number of trials included in the meta-analyses.

Sensitivity analysis according to the definition of periodontal disease was conducted by including studies with widely-accepted definition of periodontitis (Appendix L). This indicated that the results of the meta-analyses were robust in all instances. Sensitivity analysis according to the diagnostic criteria used to diagnose IBD, CD, and UC (Appendix M) could not be performed. Further sensitivity analyses were planned, but could not be conducted, due to the number and nature of the included studies

\section{Discussion}




\section{Summary of Evidence}

In this systematic review, the effect of IBD on periodontal disease and oral health was systematically assessed from eight observational clinical studies of 1297 patients. According to the results of the meta-analyses, IBD was associated with a significantly increased risk of periodontitis compared to the control group, which was more pronounced in UC rather than in CD.

IBD patients were significantly more likely to have periodontitis, had a higher percentage of sites with increased CAL, and had significantly higher mean PPD (Table 3). The included studies differed at the level of patient oral hygiene or the percentage of patients receiving anti-TNF therapy for IBD [6\% (Barros 2007) or 40\% (Vavricka et al. 2013)], but this was not reflected into considerable heterogeneity. The association between periodontitis and IBD has been attributed to common predisposing factors, such as age and genetic predisposition, as well as environmental or lifestyle factors (Indriolo et al. 2011). Smoking is strongly associated with both periodontitis (Hujoel et al. 2002) and IBD (Mahid et al. 2006), but has a distinct detrimental role for CD and a protective role for UC (Mahid et al. 2006). Although factors like socio-economic status and smoking, can confound this relationship, and indeed smokers were more heavily affected than non-smokers (Appendix D), adjusted ORs for confounders from included studies were consistent. Some studies have focused on the role of cytokines (McGee et al. 1998, Yucel-Lindberg et al. 1999) or specific pathogenic bacteria on periodontal destruction (Stein et al. 2010) and their alterations in IBD. In fact, it may be more useful to study the mechanisms explaining that the two IBD diseases are associated with periodontitis.

A combination of genetic predisposition, environmental factors and a dysbiotic microbiota with an excessive host response are main etiological factors for the initiation and progression of periodontitis and inflammatory bowel disease (Brandtzaeg 2001, Indriolo et al. 2011). The healthy gut microbiota is mainly composed of strict anaerobes and dominated by Bacteroidetes and Firmicutes. Howerever, in inflammatory bowel disease, the relative abundance of Firmicutes and the diversity of the intestinal microbiota is greatly reduced (Bull \& Plummer 2014, Forbes et al. 2016) The oral microbiota is almost as complex as that of the colon and dominated by Firmicutes, Bacteroidetes, Proteobacteria, Actinobacteria, Spirochaetes and Fusobacteria (Wade 2013). During the transition from periodontal health to periodontitis, the microbiota of periodontal pockets shifts from a predominantly Gram-positive aerobic to a predominantly Gram-negative anaerobic dominance (Khan et al. 2015). Periodontitis-associated species include Porphyromonas gingivalis, Treponema denticola, Tannerella forsythia, Aggregatibacter actinmycetemcomitans, and many others. However, Porphyromonas gingivalis, which has an impressive armamentarium of virulence factors, is 
considered a "keystone pathogen", as this bacterium can cause dysbiosis of the periodontal microbiota, which subsequently results in the destruction of the tooth-supporting tissues (Wade 2013, Khan et al. 2015).

UC patients, both smokers and non-smokers, had worse oral health than CD patients, which suggests that the response to dental plaque may be different between these groups. Indeed, UC and CD differ regarding their immunopathogenesis, which involves the T helper (Th) cell differentiation; CD is considered to be a Th1 disease while UC has characteristics of a Th2 disease (Bouma \& Strober 2003). However, a direct explorative comparison (Appendix J) indicated that included UC patients had a significantly higher plaque index than CD patients, which might have confounded this effect.

Although this was not the main scope of this systematic review, the cariological evaluation revealed that IBD had a higher DMFT index (3 studies; table 3; MD=3.85; $p<0.05)$, DMF-S index $(\mathrm{MD}=7.60 ; \mathrm{p}>0.05)$ (Grössner-Schreiber et al. 2006), more dentine caries ( $\mathrm{OR}=2.39$; $\mathrm{p}<0.05)$, higher Decayed index $(\mathrm{MD}=1.57$; $\mathrm{p}<0.05)$ (Ślebioda et al. 2011), and more oral lesions $(\mathrm{OR}=7.90 ; \mathrm{p}<0.05)$ than non-IBD patients. This is in accordance with previous reports (Sundh \& Hultén 1982, Rooney 1984) and could be attributed to nutrional behavioural changes and alterations in salivary and microbiologic conditions in the oral cavity (GrössnerSchreiber et al. 2006). CD patients have been reported to have an increased intake of refined carbohydrates compared to normal (Schütz et al. 2003) and to prefer sugary to fatty foods, due to its digestability and the concurrently less gastrointestinal symptoms (Järnerot et al. 1983), Additionally, it has been shown that although CD patients have normal saliva flow rate and buffer capacity, the number of salivary mutans streptococci and lactobacilli is higher than normal (Järnerot et al. 1983). The combination of these two findings might explain the increased caries experience of IBD patients, although other studies disagree (Halme et al. 1993, Meurman et al. 1994).

Additionally, intraoral manifestations, including oral lesions, hairy tongue, ulcers, linea alba, and recurrent aphthous stomatitis were more evident both in the composite IBD group, and in the separate CD or UC groups, compared to non-IBD patients (Tables 3; Appendix D, G, and H), which could additionally hamper oral hygiene.

\section{Strengths and Limitations}

The strengths of the present review include the pre-defined protocol and outcomes (with deviations from it noted separately in Appendix N), the extensive literature search, and the strict methodology implemented during every stage of it, according to existing evidence-based guidelines. As both university and hospital settings were 
included, no specific inclusion criteria were adopted and the included studies were observational of nature, the results could be broadly be generalized to the average patient.

Nevertheless, only a limited number of studies was identified assessing certain outcomes and some of them had uneven samples of IBD and non-IBD patients. Heterogeneity could not be explained in some cases due to the limited number of studies, while publication bias diagnostics and sensitivity analyses could not be performed, which might pose a threat to the results' validity (Papageorgiou 2014b, Papageorgiou et al. 2015c). Additionally, a large number of outcomes reported from included studies were included in the systematic review, but not in the meta-analyses, as only one study contributed to their analysis (Appendix D). It is possible that some significant differences reported by them correspond to actual differences between IBD and non-IBD patients. These can be evaluated in a future update of this review, if additional studies are included. Moreover, although this was not possible to evaluate in this review due to the limited information provided, it would be interesting to know in which cases the diagnosis of perdiodontal disease preceeds IBD or the other way around. Finally, the biggest limitation of this study is that only cross-sectional studies were included, which provide a snapshot picture of the evidence and cannot support a causality between IBD and periodontitis or vice versa.

\section{Conclusion}

According to high quality evidence originating from cross-sectional studies, inflammatory bowel disease is significantly associated with increased risk of periodontal disease. Additionally, low quality evidence from cross-sectional studies indicates that patients with inflammatory bowel disease have significantly greater caries experience compared to healthy patients.

Although considerable indications of impaired oral health in inflammatory bowel disease were found from this systematic review, this was based on cross-sectional studies of observational nature, which provide a "snapshot-picture" of a possible underlying pathology. Well-designed prospective controlled longitudinal studies of patients with/without inflammatory bowel disease and periodontitis are direly needed, in order to evaluate the impact of inflammatory bowel disease on oral health and its interrelation with tissue response to periodontic treatment. 


\section{References}

Barros, F. C. d. (2007) [Oral condition in patients with inflamatory bowel disease]. Master Thesis, Rio de Janeiro State University.

Barros, F. C. d., Brito, F., Menegat JS, Pedreira, R. R., Carvalho, A. T. P., Carneiro, A. J. d V., Zaltman, C., Fischer, R. G. \& Figueredo, C. M. S. (2008) [Prevalence of periodontitis in patients with inflammatory bowel disease]. Revista Periodontia 18, 44-8.

Beller, E. M., Glasziou, P. P., Altman, D. G., Hopewell, S., Bastian, H., Chalmers, I., Gøtzsche, P. C., Lasserson, T., Tovey, D.; PRISMA for Abstracts Group. (2013) PRISMA for abstracts: reporting systematic reviews in journal and conference abstracts. PLoS Medicine 10, e1001419.

Bouma, G. \& Strober, W. (2003) The immunological and genetic basis of inflammatory bowel disease. Nature Reviews Immunology 3, 521-533.

Brandtzaeg, P. (2001) Inflammatory bowel disease: clinics and pathology. Do inflammatory bowel disease and periodontal disease have similar immunopathogeneses? Acta Odontologica Scandinavica 59, 235-243.

Brito, F., Barros, F. C. D., Zaltman, C., Carvalho, A. T., Carneiro, A. J., Fischer, R. G., Gustafsson, A. \& Figueredo, C. M. (2008) Prevalence of periodontitis and DMFT index in patients with Crohn's disease and ulcerative colitis. Journal of Clinical Periodontology 35, 555-560.

Brito, F., Zaltman, C., Carvalho, A. T., Fischer, R. G., Persson, R., Gustafsson, A. \& Figueredo, C. M. (2013) Subgingival microflora in inflammatory bowel disease patients with untreated periodontitis. European Journal of Gastroenterology \& Hepatology 25, 239-245.

Bull, M. J. \& Plummer, N. T. (2014) Part 1: The Human Gut Microbiome in Health and Disease. Integrative Medicine (Encinitas, Calif.) 13, 17-22.

Chaffee, B. W. \& Weston, S. J. (2010) Association between chronic periodontal disease and obesity: a systematic review and meta-analysis. Journal of Periodontology 81, 1708-24.

Chan, S. W., Scully, C., Prime, S. S. \& Eveson, J. (1991) Pyostomatitis vegetans: oral manifestation of ulcerative colitis. Oral Surgery, Oral Medicine, Oral Pathology, Oral Radiology 72, 689-692.

DerSimonian, R. \& Laird, N. (1986) Meta-analysis in clinical trials. Controlled Clinical Trials 7, 177-88.

Downs, S. H. \& Black, N. (1998) The feasibility of creating a checklist for the assessment of the methodological quality both of randomised and non-randomised studies of health care interventions. Journal of Epidemiology \& Community Health 52, 377-84.

Dudeney, T. P. (1969) Crohn's disease of the mouth. Proceedings of the Royal Society of Medicine $62,1237$. 
Ellinghaus, D., Bethune, J., Petersen, B. S. \& Franke, A. (2015) The genetics of Crohn's disease and ulcerative colitis--status quo and beyond. Scandinavian Journal of Gastroenterology 50, 13-23.

Everhart, J. E. \& Ruhl, C. E. (2009) Burden of digestive diseases in the United States part I: overall and upper gastrointestinal diseases. Gastroenterology 136, 376-86.

Figueredo, C. M., Brito, F., Barros, F. C., Menegat, J. S., Pedreira, R. R., Fischer, R. G. \& Gustafsson, A. (2011) Expression of cytokines in the gingival crevicular fluid and serum from patients with inflammatory bowel disease and untreated chronic periodontitis. Journal of Periodontal Research 46, 141-146.

Forbes, J. D., Van Domselaar, G. \& Bernstein, C. N. (2016) The Gut Microbiota in Immune-Mediated Inflammatory Diseases. Frontiers in Microbiology 7, 1081.

Grössner-Schreiber, B., Fetter, T., Hedderich, J., Kocher, T., Schreiber, S. \& Jepsen, S. (2006) Prevalence of dental caries and periodontal disease in patients with inflammatory bowel disease: A case-control study. Journal of Clinical Periodontology 33, 478-484.

Guyatt, G. H., Oxman, A. D., Schünemann, H. J., Tugwell, P. \& Knottnerus, A. (2011) GRADE guidelines: a new series of articles in the Journal of Clinical Epidemiology. Journal of Clinical Epidemiology 64, 380382.

Habashneh, R. A., Khader, Y. S., Alhumouz, M. K., Jadallah, K. \& Ajlouni, Y. (2012) The association between inflammatory bowel disease and periodontitis among Jordanians: A case-control study. Journal of Periodontal Research 47, 293-298.

Halme, L., Meurman, J. H., Laine, P., von Smitten, K., Syrjänen, S., Lindqvist, C. \& Strand-Pettinen, I. (1993) Oral findings in patients with active or inactive Crohn's disease. Oral Surgery, Oral Medicine, Oral Pathology, Oral Radiology 76, 175-81.

Harty, S., Fleming, P., Rowland, M., Crushell, E., McDermott, M., Drumm, B. \& Bourke, B. (2005) A prospective study of the oral manifestations of Crohn's disease. Clinical Gastroenterology and Hepatology 3, 886-891.

Higgins, J. P. T, \& Green, S. (2011) Cochrane handbook for systemic reviews of interventions version 5.1.0 [updated March 2011]. The Cochrane Collaboration (https://www.cochrane-handbook.org).

Higgins, J. P., Thompson, S. G. \& Spiegelhalter, D. J. (2009) A re-evaluation of random-effects meta-analysis. Journal of the Royal Statistical Society: Series A (Statistics in Society) 172, 137-159.

Hujoel, P. P., Drangsholt, M., Spiekerman, C. \& DeRouen, T. A. (2002) Periodontitis-systemic disease associations in the presence of smoking--causal or coincidental? Periodontology 2000 30, 51-60. 
Indriolo, A., Greco, S., Ravelli, P. \& Fagiuoli, S. (2011) What can we learn about biofilm/host interactions from the study of inflammatory bowel disease. Journal of Clinical Periodontology 38, 36-43.

Järnerot, G., Järnmark, I. \& Nilsson, K. (1983) Consumption of refined sugar by patients with Crohn's disease, ulcerative colitis, or irritable bowel syndrome. Scandinavian Journal of Gastroenterology 18, 999-1002.

Jostins, L., Ripke, S., Weersma, R. K., Duerr, R. H., McGovern, D. P., Hui, K. Y., Lee, J. C., Schumm, L. P., Sharma, Y., Anderson, C. A., Essers, J., Mitrovic, M., Ning, K., Cleynen, I., Theatre, E., Spain, S. L., Raychaudhuri, S., Goyette, P., Wei, Z., Abraham, C., Achkar, J. P., Ahmad, T., Amininejad, L., Ananthakrishnan, A. N., Andersen, V., Andrews, J. M., Baidoo, L., Balschun, T., Bampton, P. A., Bitton, A., Boucher, G., Brand, S., Büning, C., Cohain, A., Cichon, S., D'Amato, M., De Jong, D., Devaney, K. L., Dubinsky, M., Edwards, C., Ellinghaus, D., Ferguson, L. R., Franchimont, D., Fransen, K., Gearry, R., Georges, M., Gieger, C., Glas, J., Haritunians, T., Hart, A., Hawkey, C., Hedl, M., Hu, X., Karlsen, T. H., Kupcinskas, L., Kugathasan, S., Latiano, A., Laukens, D., Lawrance, I. C., Lees, C. W., Louis, E., Mahy, G., Mansfield, J., Morgan, A. R., Mowat, C., Newman, W., Palmieri, O., Ponsioen, C. Y., Potocnik, U., Prescott, N. J., Regueiro, M., Rotter, J. I., Russell, R. K., Sanderson, J. D., Sans, M., Satsangi, J., Schreiber, S., Simms, L. A., Sventoraityte, J., Targan, S. R., Taylor, K. D., Tremelling, M., Verspaget, H. W., De Vos, M., Wijmenga, C., Wilson, D. C., Winkelmann, J., Xavier, R. J., Zeissig, S., Zhang, B., Zhang, C. K., Zhao, H.; International IBD Genetics Consortium (IIBDGC), Silverberg, M. S., Annese, V., Hakonarson, H., Brant, S. R., Radford-Smith, G., Mathew, C. G., Rioux, J. D., Schadt, E. E., Daly, M. J., Franke, A., Parkes, M., Vermeire, S., Barrett, J. C. \& Cho, J. H. (2012) Host-microbe interactions have shaped the genetic architecture of inflammatory bowel disease. Nature 491, 119-24.

Khan, S. A., Kong, E. F., Meiller, T. F. \& Jabra-Rizk, M. A. Periodontal Diseases: Bug Induced, Host Promoted. PLoS Pathogens 11, e1004952.

Kinane, D. F. \& Bartold, P. M. (2007) Clinical relevance of the host responses of periodontitis. Periodontology $200043,278-293$.

Koutsochristou, V., Zellos, A., Dimakou, K., Panayotou, I., Siahanidou, S., Roma-Giannikou, E. \& Tsami, A. (2015) Dental Caries and Periodontal Disease in Children and Adolescents with Inflammatory Bowel Disease: A Case-Control Study. Inflammatory Bowel Diseases 21, 1839-46.

Liberati, A., Altman, D. G., Tetzlaff, J., Mulrow, C., Gøtzsche, P. C., Ioannidis, J. P., Clarke, M., Devereaux, P. J., Kleijnen, J. \& Moher, D. (2009) The PRISMA statement for reporting systematic reviews and metaanalyses of studies that evaluate health care interventions: explanation and elaboration. Journal of Clinical Epidemiology 62, e1-34. 
Mahid, S. S., Minor, K. S., Soto, R. E., Hornung, C. A. \& Galandiuk, S. (2006) Smoking and inflammatory bowel disease: a meta-analysis. Mayo Clinic Proceedings 81, 1462-71.

McGee, J. M., Tucci, M. A., Edmundson, T. P., Serio, C. L. \& Johnson, R. B. (1998) The relationship between concentrations of proinflammatory cytokines within gingiva and the adjacent sulcular depth. Journal of Periodontology 69, 865-871.

Meurman, J. H., Halme, L., Laine, P., von Smitten, K. \& Lindqvist, C. (1994) Gingival and dental status, salivary acidogenic bacteria, and yeast counts of patients with active or inactive Crohn's disease. Oral Surgery, Oral Medicine, Oral Pathology, Oral Radiology 77, 465-8.

Molodecky, N. A., Soon, I. S., Rabi, D. M., Ghali, W. A., Ferris, M., Chernoff, G., Benchimol, E. I., Panaccione, R., Ghosh, S., Barkema, H. W. \& Kaplan, G. G. (2012) Increasing incidence and prevalence of the inflammatory bowel diseases with time, based on systematic review. Gastroenterology 142, 46-54.e42.

Neovius, M., Arkema, E. V., Blomqvist, P., Ekbom, A. \& Smedby, K. E. (2013) Patients with ulcerative colitis miss more days of work than the general population, even following colectomy. Gastroenterology 144, $536-43$.

Nibali, L., Tatarakis, N., Needleman, I., Tu, Y. K., D'Aiuto, F., Rizzo, M. \& Donos, N. (2013) Clinical review: Association between metabolic syndrome and periodontitis: a systematic review and meta-analysis. The Journal of Clinical Endocrinology and Metabolism 98, 913-20.

Papageorgiou, S. N. (2014a) Meta-analysis for orthodontists: Part I--How to choose effect measure and statistical model. Journal of Orthodontics 41, 317-326.

Papageorgiou, S. N. (2014b) Meta-analysis for orthodontists: Part II--Is all that glitters gold? Journal of Orthodontics 41, 327-336.

Papageorgiou, S. N., Antonoglou, G. N., Tsiranidou E, Jepsen, S. \& Jäger, A. (2014) Bias and small-study effects influence treatment effect estimates: a meta-epidemiological study in oral medicine. Journal of Clinical Epidemiology 67, 984-992.

Papageorgiou, S. N., Kloukos, D., Petridis, H. \& Pandis, N. (2015c) Publication of statistically significant research findings in prosthodontics \& implant dentistry in the context of other dental specialties. Journal of Dentistry 43, 1195-1202.

Papageorgiou, S. N., Reichert, C., Jäger, A. \& Deschner, J. (2015a) Effect of overweight/obesity on response to periodontal treatment: systematic review and a meta-analysis. Journal of Clinical Periodontology 42, 24761. 
Papageorgiou, S. N., Xavier, G. M. \& Cobourne, M. T. (2015b) Basic study design influences the results of orthodontic clinical investigations. Journal of Clinical Epidemiology 68, 1512-1522.

Petersen, P. E. \& Ogawa, H. (2012) The global burden of periodontal disease: towards integration with chronic disease prevention and control. Periodontology 2000 60, 15-39.

Rathnayake, N. (2008) Pro-inflammatory mediators in gingival crevicular fluid of patients with Inflammatory Bowel Disease, IBD. Master Thesis, Karolinska Institutet.

Rooney, T. P. (1984) Dental caries prevalence in patients with Crohn's disease. Oral Surgery, Oral Medicine, Oral Pathology, Oral Radiology 57, 623-4.

Scheper, H. J. \& Brand, H. S. (2002) Oral aspects of Crohn's disease. International Dental Journal 52, 163172.

Schütz, T., Drude, C., Paulisch, E., Lange, K. P. \& Lochs, H. (2003) Sugar intake, taste changes and dental health in Crohn's disease. Digestive Diseases (Basel, Switzerland) 21, 252-7.

Silva, F. d. B. (2008) [Th cell profile in the gingival crevicular fluid from inflammatory bowel disease patients with chronic periodontitis]. Ph.D. Dissertation, Rio de Janeiro State University.

Ślebioda, Z., Szponar, E. \& Linke, K. (2011) Comparative analysis of the oral cavity status in patients with Crohn's disease and ulcerative colitis. Journal of Stomatology 64, 212-224.

Sloan, J., Symonds, T., Vargas-Chanes, D. \& Fridley, B. (2006) Practical guidelines for assessing the clinical significance of health-related quality of life changes within clinical trials. Drug Information Journal 37, 2331.

Stein, J. M., Lammert, F., Zimmer, V., Granzow, M., Reichert, S., Schulz, S., Ocklenburg, C. \& Conrads, G. (2010) Clinical periodontal and microbiologic parameters in Patients With Crohn's Disease With Consideration of the CARD15 Genotype. Journal of Periodontology 81, 535-545.

Sundh, B. \& Emilson, C. G. (1989) Salivary and microbial conditions and dental health in patients with Crohn's disease: a 3-year study. Oral Surgery, Oral Medicine, Oral Pathology, Oral Radiology 67, 286-90.

Sundh, B. \& Hultén, L. (1982) Oral status in patients with Crohn's disease. Acta chirurgica Scandinavica 148, $531-4$.

Suvan, J., D'Aiuto, F., Moles, D. R., Petrie, A. \& Donos, N. (2011) Association between overweight/obesity and periodontitis in adults. A systematic review. Obesity Reviews 12, e381-404. 
Van Dyke, T. E., Dowell, V. R. Jr., Offenbacher, S., Snyder, W. \& Hersh, T. (1986) Potential role of microorganisms isolated from periodontal lesions in the pathogenesis of inflammatory bowel disease. Infection and Immunity 53, 671-677.

Vavricka, S. R., Manser, C. N., Hediger, S., Vögelin, M., Scharl, M., Biedermann, L., Rogler, S., Seibold, F., Sanderink, R., Attin, T., Schoepfer, A., Fried, M., Rogler, G. \& Frei, P. (2013) Periodontitis and gingivitis in inflammatory bowel disease: a case-control study. Inflammatory Bowel Diseases 19, 2768-2777.

Wade, W. G. (2013) The oral microbiome in health and disease. Pharmacological Research 69, 137-143.

Yucel-Lindberg, T., Nilsson, S. \& Modeer, T. (1999) Signal transduction pathways involved in the synergistic stimulation of prostaglandin production by interleukin-1b and tumor necrosis factor a in human gingival fibroblast. Journal of Dental Research 78, 61-68.

Zervou, F., Gikas, A., Merikas, E., Peros, G., Sklavaina, M., Loukopoulos, J. \& Triantafillidis, J. K. (2007) Oral manifestations of patients with inflammatory bowel disease. Annals of Gastroenterology 17, 1108-7471. 
Tables

Table 1. Characteristics of included studies

\begin{tabular}{|c|c|c|c|c|c|c|c|c|}
\hline Nr. & Study & $\begin{array}{l}\text { Design; setting; } \\
\text { language }\end{array}$ & Patients (no; M/F; mean age) & $\begin{array}{l}\text { Extras (ethnicity; smoking; } \\
\text { hypertension; diabetes) }\end{array}$ & IBD & Perio & Oral & Inter \\
\hline 1 & $\begin{array}{l}\text { Barros } \\
\text { collated }^{\$}\end{array}$ & $\begin{array}{l}\text { CC; University; } \\
\text { Brazil; EN/POR }\end{array}$ & $\begin{array}{l}\text { IBD: } 179(64 / 115) \text { pts; } 40.9 \text { yrs } \\
\text { Ctr: } 74 \text { (24/50) pts; } 40.3 \text { yrs }\end{array}$ & $\begin{array}{l}\text { 53\% Caucasian, } 37 \% \text { mixed, } \\
9 \% \text { African; } 38 \% \text { smokers; } \\
14 \% \text { hypertension; } 1 \% \\
\text { diabetes }\end{array}$ & $\begin{array}{l}\text { CD: } 99 \text { (31/68) pts; } 39.0 \text { yrs; } 22 \% \\
\text { active; extraintestinal } 51 \% \\
\text { UC: } 80 \text { (33/47) pts; } 43.3 \text { yrs; } 24 \% \\
\text { active; extraintestinal } 53 \%\end{array}$ & $\begin{array}{l}\text { Periodontitis (at least } 4 \mathrm{x} \\
\text { CAL } \geq 3 \mathrm{~mm}) ; \% \text { PI; \%BOP; } \\
\text { PPD; CAL; \%CAL } \geq 3 \mathrm{~mm} \text {; IL- } \\
\text { 18; IL-1 } \beta \text {; IL-4; IL-6; IFN- } \gamma \text {; } \\
\text { various bacteria }\end{array}$ & $\begin{array}{l}\text { \#Teeth; } \\
\text { DMFT; }\end{array}$ & $\begin{array}{l}91 \% \text { of } \mathrm{CD} \text { pts } \\
\text { and } 100 \% \text { of UC } \\
\text { pts receiving } \\
\text { medication }\end{array}$ \\
\hline 2 & $\begin{array}{l}\text { Groessner- } \\
\text { Schreiber et al. } \\
\text { (2006) }\end{array}$ & $\begin{array}{l}\text { CC; } \\
\text { University/private } \\
\text { practice; } \\
\text { Germany; EN }\end{array}$ & $\begin{array}{l}\text { IBD: } 62(24 / 38) \mathrm{pts} ; 38.4 \mathrm{yrs} \\
\text { Ctr: } 59 \text { (24/35) pts; } 38.2 \text { yrs }\end{array}$ & $\begin{array}{l}\text { 100\% Caucasian; } 45 \% \\
\text { smokers }\end{array}$ & $\begin{array}{l}\text { CD: } 46 \text { pts; } 39 \% \text { active } \\
\text { UC: } 16 \text { pts; } 25 \% \text { active } \\
\text { Overall }\end{array}$ & $\begin{array}{l}\% \mathrm{PI} ; \% \mathrm{BOP} ; \mathrm{PPD} \\
\% \mathrm{CAL} \geq 4 \mathrm{~mm} ; \% \mathrm{CAL} \geq 5 \mathrm{~mm}\end{array}$ & $\begin{array}{l}\text { \#teeth; } \\
\text { DMFS; } \\
\text { dentine } \\
\text { caries }\end{array}$ & $\begin{array}{l}\text { All IBD } \\
\text { receiving } \\
\text { medication }\end{array}$ \\
\hline 3 & $\begin{array}{l}\text { Habashneh et } \\
\text { al. (2012) }\end{array}$ & $\begin{array}{l}\text { CC; University; } \\
\text { Jordan; EN }\end{array}$ & $\begin{array}{l}\text { IBD: } 160 \text { (94/66) pts; NR } \\
\text { Ctr: } 100 \text { (62/38) pts; NR }\end{array}$ & $\begin{array}{l}70 \% \text { smokers; } 11 \% \\
\text { hypertension }\end{array}$ & $\begin{array}{l}\text { CD: } 59(33 / 26) \mathrm{pts} \\
\text { UC: } 101(61 / 40) \mathrm{pts}\end{array}$ & $\begin{array}{l}\text { Periodontitis; PI; GI; PPD; } \\
\text { CAL; GR; \%BOP; \%PPD } \geq 3 \text {; } \\
\text { \%PPD } \geq 4 ; \% \mathrm{CAL} \geq 3 ; \\
\% \mathrm{CAL} \geq 4 ; \% \mathrm{CAL} \geq 5\end{array}$ & - & - \\
\hline 4 & $\begin{array}{l}\text { Koutsochristou } \\
\text { et al. }(2015\end{array}$ & $\begin{array}{l}\text { CC; University; } \\
\text { Greecel EN }\end{array}$ & $\begin{array}{l}\text { IBD: } 55(25 / 30) \mathrm{pts} ; 12.3 \mathrm{yrs} \\
\text { Ctr: } 55(25 / 30) \mathrm{pts} ; 12.2 \mathrm{yrs}\end{array}$ & $0 \%$ smokers & $\begin{array}{l}\text { CD: } 36(18 / 18) \mathrm{pts} \\
\text { UC: } 19(7 / 12) \mathrm{pts}\end{array}$ & $\begin{array}{l}\text { Periodontal treatment need } \\
\text { (CPITN 2-3); \%GI; \%PI }\end{array}$ & DMFT & $\begin{array}{l}\text { All IBD } \\
\text { receiving } \\
\text { medication }\end{array}$ \\
\hline 5 & $\begin{array}{l}\text { Rathnayake } \\
(2008)\end{array}$ & $\begin{array}{l}\text { CC; University; } \\
\text { Sweden; EN }\end{array}$ & $\begin{array}{l}\text { IBD: } 30(15 / 15) \text { pts; } 41.6 \text { yrs } \\
\text { Ctr: } 15 \text { (7/8) pts; } 42.1 \text { yrs }\end{array}$ & $\begin{array}{l}49 \% \text { Caucasian, } 44 \% \text { mixed, } \\
7 \% \text { black; } 13 \% \text { smokers; } 13 \% \\
\text { hypertension; } 0.2 \% \text { diabetes }\end{array}$ & $\begin{array}{l}\text { CD: } 15(8 / 7) \text { pts; } 38.2 \text { yrs } \\
\text { UC: } 15(7 / 8) \text { pts; } 45.0 \text { yrs } \\
31 \% \text { extra-intestinal } \\
\text { manifestations; } 18 \% \text { active } \\
\text { disease }\end{array}$ & $\begin{array}{l}\text { \%PI; \%BOP; PPD; CAL; } \\
\text { \%CAL } \geq 3 \mathrm{~mm} ; \% \mathrm{CAL} \geq 4 \mathrm{~mm} ; \\
\text { GCF volume; IL-18; IL-12; }\end{array}$ & \#teeth; & $\begin{array}{l}96 \% \text { receiving } \\
\text { medications }\end{array}$ \\
\hline 6 & $\begin{array}{l}\text { Ślebioda et al. } \\
\text { (2011) }\end{array}$ & $\begin{array}{l}\text { CC; University; } \\
\text { Poland; EN/PL }\end{array}$ & $\begin{array}{l}\text { IBD: } 95 \text { (53/42) pts; } 37.3 \mathrm{yrs} \\
\text { Ctr: } 70(24 / 46) \mathrm{pts} ; 31.6 \mathrm{yrs}\end{array}$ & No systematic diseases & $\begin{array}{l}\text { CD: } 70(37 / 33) \mathrm{pts} ; 37.4 \mathrm{yrs} \\
\text { UC: } 25 \text { (16/9) pts; } 37.2 \mathrm{yrs}\end{array}$ & $\begin{array}{l}\text { Periodontal treatment need } \\
\text { (CPITN 2-3) }\end{array}$ & $\begin{array}{l}\text { \#teeth; } \\
\text { DMFT; } \\
\text { OHI }\end{array}$ & - \\
\hline 7 & $\begin{array}{l}\text { Van Dyke et } \\
\text { al. }(2013)^{*}\end{array}$ & $\begin{array}{l}\text { CC; University; } \\
\text { USA; EN }\end{array}$ & $\begin{array}{l}\text { IBD:20 (NR) pts; NR yrs } \\
\text { Ctr: } 20 \text { (NR) pts; NR yrs }\end{array}$ & Smoking up to $10 \mathrm{cg} / \mathrm{day}$ & $\begin{array}{l}\text { (Only for the IBD \& PD } \\
\text { subgroup) } \\
\text { CD: } 6 \text { (NR) pts; NR yrs } \\
\text { UC } 4 \text { (NR) pts; NR yrs }\end{array}$ & $\begin{array}{l}\text { Redness; BOP; CAL; CF- } \\
\text { PGE2 levels }\end{array}$ & - & $\begin{array}{l}50 \% \text { taking } \\
\text { steroids }\end{array}$ \\
\hline 8 & $\begin{array}{l}\text { Vavricka et al. } \\
\text { (2013) }\end{array}$ & $\begin{array}{l}\text { CC; University; } \\
\text { Switzerland; EN }\end{array}$ & $\begin{array}{l}\text { IBD: } 113(65 / 48) \text { pts; } 40.6 \text { yrs } \\
\text { Ctr: } 113(58 / 55) \text { pts; } 41.7 \text { yrs }\end{array}$ & $58 \%$ smokers & $\begin{array}{l}\text { CD: } 69 \text { (37/32) pts; } 39.6 \mathrm{yrs} \\
\text { UC: } 44 \text { (28/16) pts; } 42.3 \mathrm{yrs}\end{array}$ & $\begin{array}{l}\text { PPD; CAL; CAL at deepest } \\
\text { pocket; \%BOP; \%PBI }\end{array}$ & DMFT & $\begin{array}{l}\text { About } 73 \% \\
\text { receiving } \\
\text { medication and } \\
36 \% \text { had surgery }\end{array}$ \\
\hline 9 & $\begin{array}{l}\text { Zervou et al. } \\
\text { (2007) }\end{array}$ & $\begin{array}{l}\text { CC; state hospital; } \\
\text { Greece; EN }\end{array}$ & $\begin{array}{l}\text { IBD: } 30 \text { (NR) pts; } 40.0 \text { yrs } \\
\text { Ctr: } 47 \text { (NR) pts; } 43.0 \text { yrs }\end{array}$ & - & $\begin{array}{l}\text { CD: } 15 \mathrm{pts} \\
\text { UC: } 15 \mathrm{pts} \\
\text { Overall } 67 \% \text { active }\end{array}$ & $\begin{array}{l}\text { Periodontitis; gingivitis; } \\
\text { gingival bleeding }\end{array}$ & - & $\begin{array}{l}80 \% \text { receiving } \\
\text { medication }\end{array}$ \\
\hline
\end{tabular}

*various non-periodontal outcomes relating to IBD microbiota, neutrophil chemotaxis, phagocytosis, inhibition of neutrophil function were omitted.

\$Including 6 studies (Barro 2007, Barros et al. 2008, Brito et al. 2008, Silva 2008, Figueredo et al. 2011, Brito et al. 2013).

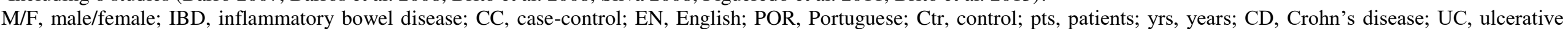

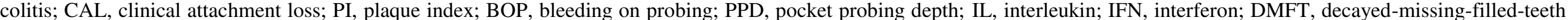

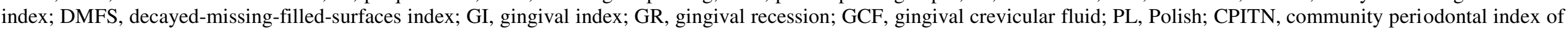
treatment need; OHI, oral health index; NR, not reported; CF-PGE2, prostaglandin E2 in gingival crevicular fluid; PBI, papilla bleeding index. 


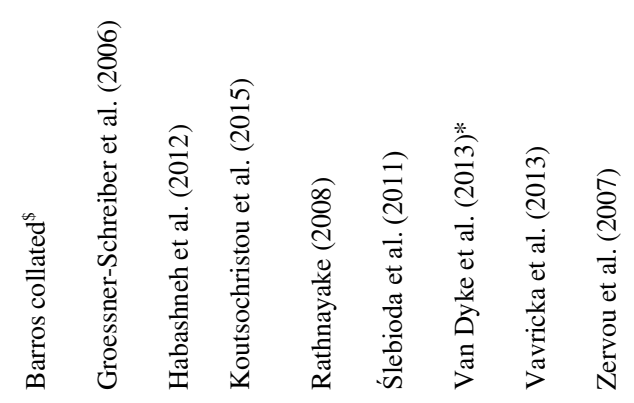

$\stackrel{3}{?}$

Was selection of IBD and non-IBD patients drawn from the same population and over the same period?

Can we be confident that IBD patients had IBD?

Can we be confident that non-IBD patients had no IBD?

Can we be confident that presence/absence of periodontal disease was not used as a patient selection criterion?

Are the characteristics of the patients included in the study clearly described ?

$\gg$ Did the study match IBD and non-IBD patients for all variables that are associated with periodontal health (age, gender, smoking, diabetes) or did the statistical analysis adjust for these prognostic variables?

Can we be confident in the assessment of periodontal disease (outcome measures valid and reliable)?

Is reporting of results complete and transparent (for continuous outcomes: n, mean, SD; for binary outcomes: sample and events; for calculated effect sizes: poin estimate and SE or $95 \% \mathrm{CI}$ )

$\stackrel{\infty}{g}$

Were the statistical tests used to assess the main outcomes appropriate (normality/pairness)?

Have actual probability values been reported (e.g. 0.035 rather than $<0.05$ ) for the main outcomes except where the probability value is less than 0.001 ?

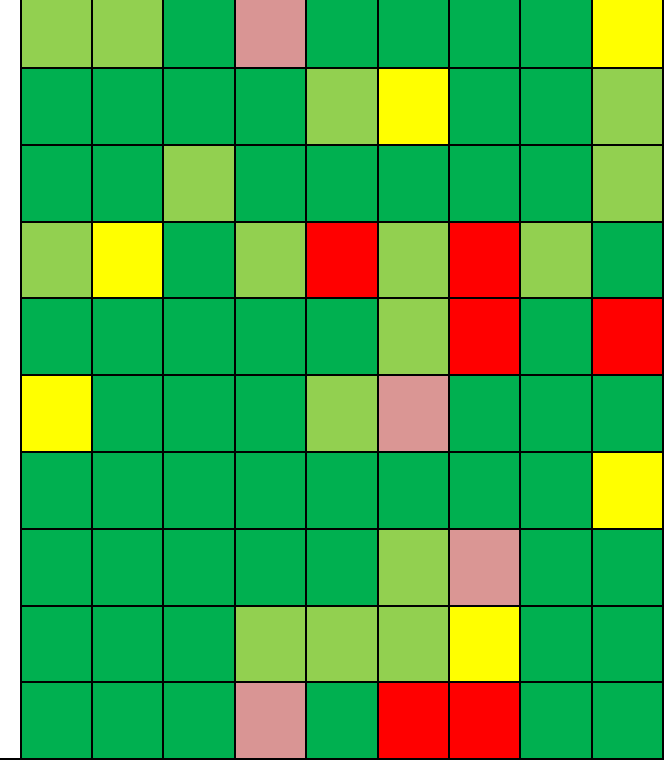

Definitely yes (low risk of bias)
Probably yes
Not possible (unclear)
Probably no

${ }^{\$}$ Including 6 studies (Barro 2007, Barros et al. 2008, Brito et al. 2008, Silva 2008, Figueredo et al. 2011, Brito et al. 2013). 
Table 3. Results of meta-analyses comparing patients with inflammatory bowel disease (combined Crohn's disease and ulcerative colitis) compared to healthy patients

\begin{tabular}{|c|c|c|c|c|c|c|}
\hline \multirow[b]{2}{*}{ Outcomes } & \multirow[b]{2}{*}{ Studies } & \multicolumn{3}{|c|}{ Effect size } & \multicolumn{2}{|c|}{ Heterogeneity } \\
\hline & & $\mathrm{MD}, \mathrm{SMD}$, or OR $(95 \% \mathrm{CI})$ & $\mathrm{p}$ & $95 \% \operatorname{PrI}$ & $\mathrm{I}^{2}(95 \% \mathrm{CI})$ & $\tau^{2}$ \\
\hline \multicolumn{7}{|l|}{ Primary outcome } \\
\hline Periodontitis & 4 & OR: $4.55(3.00,6.91)$ & $<0.001$ & $1.83,11.36$ & $0(0,68)$ & 0 \\
\hline \multicolumn{7}{|l|}{ Secondary outcomes } \\
\hline Number of teeth & 4 & MD: $-1.00(-2.15,0.16)$ & 0.090 & $-5.30,3.31$ & $48(0,81)$ & 0.656 \\
\hline DMFT index & $4^{\dagger}$ & MD: $3.85(2.36,5.34)$ & $<0.001$ & $-2.54,10.24$ & $74(0,89)$ & 1.626 \\
\hline Plaque index & $\#$ & & & & & \\
\hline Gingival index & 2 & SMD: $1.19(0.75,1.63)$ & $<0.001$ & NA & 71 (NA) & 0.073 \\
\hline Bleeding on probing & 4 & MD: $1.90(-3.21,7.01)$ & 0.466 & $-19.10,22.91$ & $69(0,87)$ & 17.033 \\
\hline Clinical attachment loss & a & & & & & \\
\hline Clinical attachment loss $>3 \mathrm{~mm}$ & 2 & MD: $8.88(1.48,16.27)$ & 0.019 & NA & $52(\mathrm{NA})$ & 15.535 \\
\hline Clinical attachment loss $>4 \mathrm{~mm}$ & 2 & MD: $11.52(1.82,21.23)$ & 0.020 & NA & 68 (NA) & 34.435 \\
\hline Clinical attachment loss $>5 \mathrm{~mm}$ & 2 & MD: $3.72(1.84,5.60)$ & $<0.001$ & NA & 0 (NA) & 0 \\
\hline Pocket probing depth & $3^{\mathrm{b}, *}$ & MD: $0.63(0.16,1.10)$ & 0.008 & $-5.37,6.63$ & $97(94,98)$ & 0.165 \\
\hline Pocket probing depth $>3 \mathrm{~mm}$ & 2 & MD: $0.10(-2.75,2.94)$ & 0.948 & NA & 0 (NA) & 0 \\
\hline Pocket probing depth $>4 \mathrm{~mm}$ & $3^{\mathrm{c}, *}$ & MD: $-3.45(-10.46,3.55)$ & 0.334 & $-88.64,81.74$ & 94 (NA) & 32.178 \\
\hline Periodontal treatment need (CPITN 2-3) & 2 & OR: $6.10(2.50,14.93)$ & $<0.001$ & NA & 60 (NA) & 0.255 \\
\hline Oral lesions & $3 *$ & OR: $7.90(1.46,42.61)$ & 0.016 & $0,>10^{9}$ & $93(80,96)$ & 1.756 \\
\hline Angular cheilitis & 2 & OR: $8.26(0.53,128.31)$ & 0.131 & NA & 65 (NA) & 2.635 \\
\hline Aphthous lesions & 3 & OR: $2.45(1.18,5.12)$ & 0.017 & $0.00,1829.56$ & $29(0,80)$ & 0.130 \\
\hline Hairy tongue & 2 & OR: $8.01(0.97,66.33)$ & 0.054 & NA & 0 (NA) & 0 \\
\hline Ulceration & 2 & OR: $9.10(1.12,74.28)$ & 0.039 & NA & 0 (NA) & 0 \\
\hline
\end{tabular}

MD, mean difference; OR, odds ratio; CI, confidence interval; PrI, predictive interval; NA, not applicable; DMFT, decayed missing filling teeth; CPITN, Community Periodontal Index of Treatment Needs.

"Standardized Mean Difference (SMD) used instead of MD to combine two different plaque indeces (O Leary index and Sillness index); meta-analysis omitted due to heterogeneity: 5 studies highly dispersed on both sides ( $\mathrm{SMD}=0.12 ; 95 \% \mathrm{CI}=-0.29,0.52 ; \mathrm{P}=0.579 ; \mathrm{I}^{2}=86 \%$ ); omission of a single trial not feasible.

*extreme heterogeneity, but agrees on direction; effect magnitude might be imprecise.

$\dagger$ moderate to high heterogeneity, but agrees on direction and the calculated inconsistency $\left(\mathrm{I}^{2}\right)$ has large uncertainy interval $0 \%$ to $89 \%$; meta-analysis judged as safe

${ }^{a}$ Meta-analysis omitted; three studies with extreme heterogeneity; studies evenly distributed $\left(\mathrm{MD}=0.23 ; 95 \% \mathrm{CI}=-0.25,0.71 ; \mathrm{p}=0.349 ; \mathrm{I}^{2}=87\right)$.

${ }^{\mathrm{b}}$ Omitted study of Groessner-Schreiber et al. (2006) due to homogeneity; results might be unstable (original: $\left.\mathrm{MD}=0.46 ; 95 \% \mathrm{CI}=0.04,0.87 ; \mathrm{p}=0.030 ; \mathrm{I}^{2}=97\right)$.

${ }^{c}$ Omitted study of of Groessner-Schreiber et al. (2006) due to homogeneity; results might be unstable (original: $\left.\mathrm{MD}=-2.20 ; 95 \% \mathrm{CI}=-7.31,2.91 ; \mathrm{p}=0.399 ; \mathrm{I}^{2}=90\right)$. 
Table 4. Summary of findings table with the risk of bias assessment across studies (GRADE approach)

Patients: patients of any age, sex or ethnicity

Settings: university clinics (Brazil, Greece, Jordan, Switzerland) and state hospital (Greece)

Intervention (exposure): inflammatory bowel disease (Crohn's disease and ulcerative colitis)

Comparison: control (no inflammatory bowel disease)

Outcomes Illustrative comparative risks/changes $(95 \% \mathrm{CI})$

IBD-free patients

Patients

GRADE

Comments

Assumed risk per 1000 patients (range)

IBD patients

Corresponding risk per 1000 patients $(95 \% \mathrm{CI})$

Periodontitis

IBD

468 patients per 1000 (range 0 to 797)

332 more patients per 1000 ( 257 to 388 more) compared

to healthy patients.

$\operatorname{High}^{1,2}$

$\bigoplus \oplus \oplus \oplus$

$\mathrm{NNT}=4(3$ to 4$)$

$\mathrm{CD}$

309 more patients per 1000 (211 to 384 more) compared to healthy patients.

$\mathrm{High}^{2,3}$

$\bigoplus \bigoplus \bigoplus \bigoplus$

$\mathrm{NNT}=4$ (3 to 5$)$

351 more patients per 1000 ( 252 to 421 more) compared

508 (3) High $^{1,2}$

to healthy patients.

$\oplus \oplus \oplus \oplus \quad \mathrm{NNT}=3$ (3 to 4$)$

UC

Assumed levels (range)

Corresponding levels (95\% CI)

Number of teeth

IBD

25.14 teeth (range 23.70 to 26.72 )

Decrease by 1.00 teeth ( 2.15 decrease to 0.16 increase)

compared to healthy patients.

Decrease by 0.11 teeth ( 1.52 decrease to 1.31 increase)

compared to healthy patients.

$\oplus \oplus \ominus \ominus$

$\mathrm{CD}$

Decrease by 1.86 teeth ( 0.04 to 3.68 decrease) compared

to healthy patients.

$\oplus \oplus \ominus \ominus$

$\mathrm{UC}$

Increase by 3.85 ( 2.36 to 5.34 increase) compared to healthy patients

Increase by 3.73 ( 0.70 to 6.75 increase) compared to

healthy patients

Increase by 3.97 (1.94 to 6.00 increase) compared to

healthy patients

$381(3) \quad$ Low

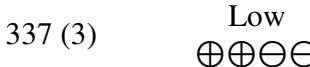

DMFT index

IBD

7.70 (range 2.04 to 12.50 )

$\mathrm{UC}$

All judgements start from "low", due to the vast inclusion of non-randomized studies.

${ }^{1}$ Rated up by two levels for very large effect included in the confidence and predictive interval.

${ }^{2}$ Rated up by one; estimates adjusted for confounders confirmed results agree with original analyses.

${ }^{3}$ Rated up by one level for large effect included in the confidence and predictive interval.

$\mathrm{CI}$, confidence interval; IBD, inflammatory bowel disease; NNT, number needed to treat; $\mathrm{CD}$, Crohn's disease; UC, ulcerative colitis. 
Figure Legends

Fig. 1. Flow diagram that depicts the workflow of identifying qualifying studies.
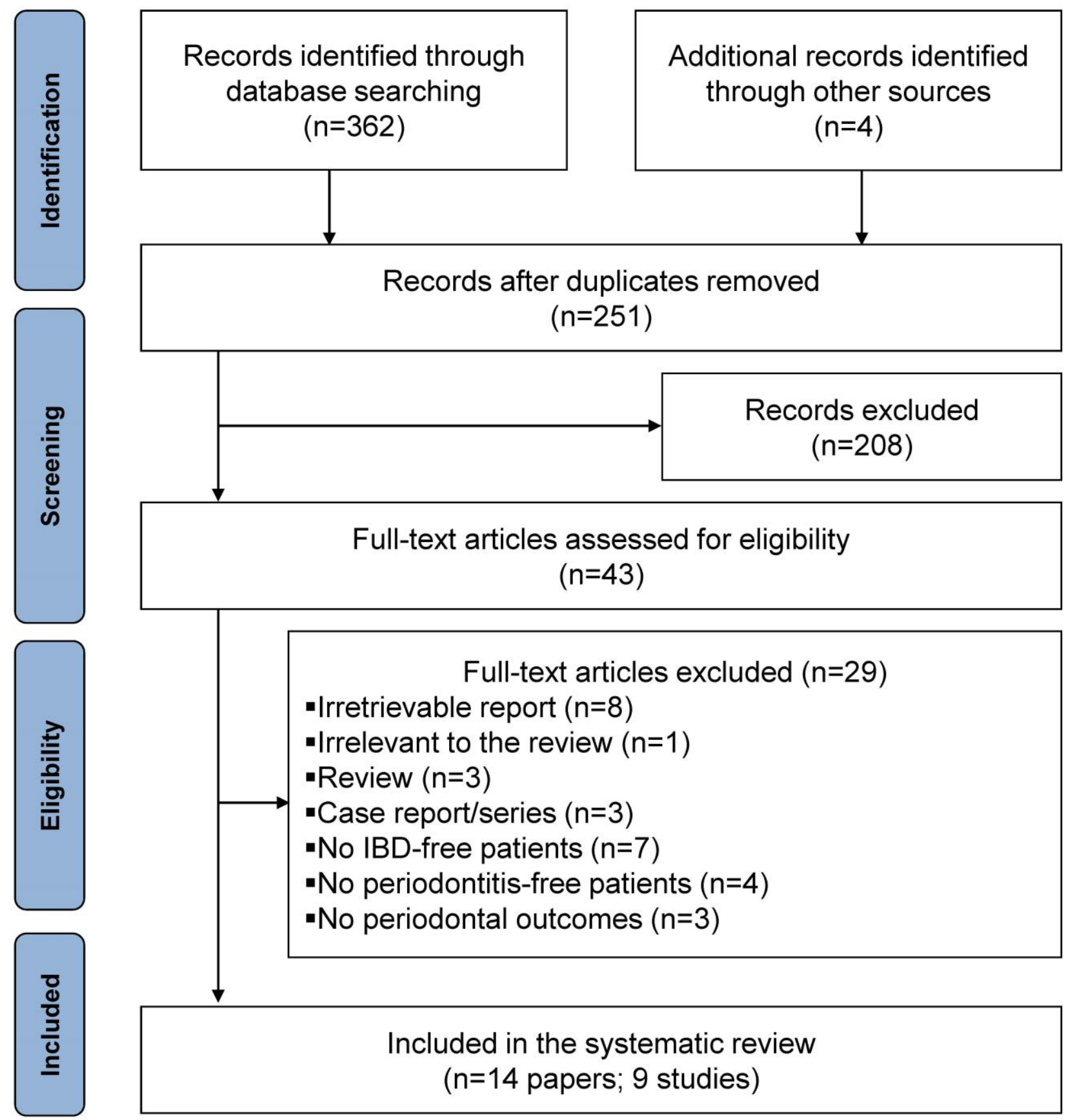
Fig. 2. Forest plot with the meta-analyses regarding the prevalence of periodontitis. IBD, inflammatory bowel disease; n, affected patients; N, sample; OR, odds ratio; CI, confidence interval; adj, adjusted estimates; PrI, predictive interval; CD, Crohn's disease; UC, ulcerative colitis.

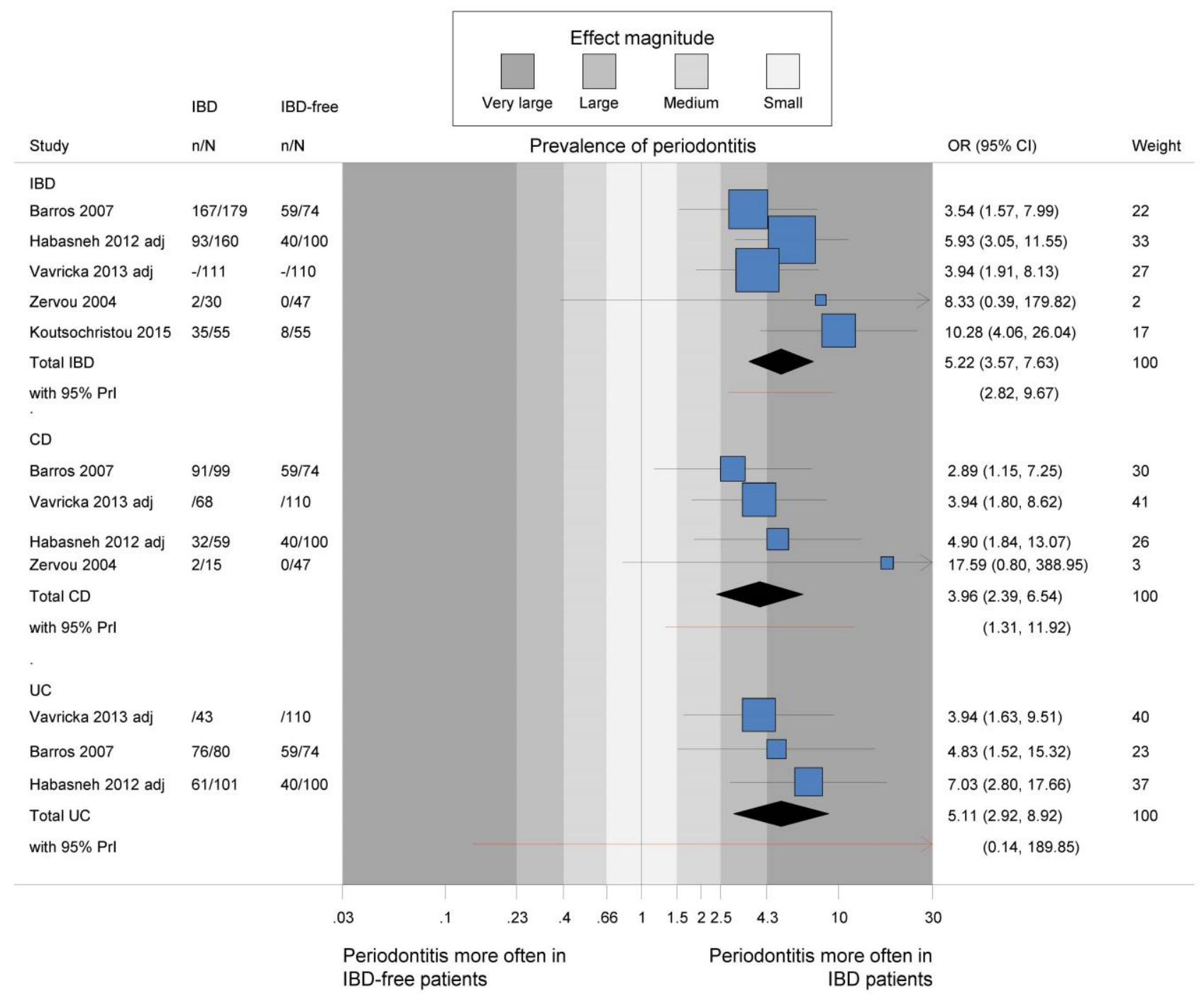




\section{Inflammatory bowel disease and oral health: systematic review and a meta-analysis}

\section{Supplementary Data}

Appendix S1 - Eligibility criteria used in this systematic review according to the PICOS approach.

\begin{tabular}{|c|c|c|}
\hline Characteristic & Inclusion criteria & Exclusion criteria \\
\hline \multicolumn{3}{|c|}{ Observational analysis (prevalence of periodontitis) } \\
\hline Patients & Patients of any age or sex & 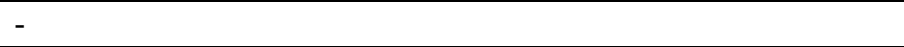 \\
\hline $\begin{array}{l}\text { Intervention } \\
\text { (here } \\
\text { exposure) }\end{array}$ & Diagnosis of some type of IBD. & No patients with some type of IBD. \\
\hline Comparison & Absence of IBD. & No comparison with IBD-free patients. \\
\hline Outcome & $\begin{array}{l}\text { Periodontal health. } \\
\text { Primary outcome: periodontitis. } \\
\text { Secondary outcomes: any outcome pertaining to (a) clinical periodontal outcome, } \\
\text { (b) markers of periodontal inflammation. }\end{array}$ & Absence of periodontal outcomes. \\
\hline Study design & $\begin{array}{l}\text { Studies of observational nature are eligible for this analysis. If provided, } \\
\text { prevalence data will be also included from RCTs. Any clinical setting will be } \\
\text { included. }\end{array}$ & Animal studies and non-clinical studies will be excluded. \\
\hline \multicolumn{3}{|c|}{ Interventional analysis (effect of periodontal or IBD treatment) } \\
\hline Characteristic & Inclusion criteria & Exclusion criteria \\
\hline Patients & Patients of any age or sex with IBD and/or periodontal disease. & - \\
\hline Intervention & Any kind of intervention addressing the periodontal disease or the IBD. & $\begin{array}{l}\text { No intervention addressing the periodontal disease or the } \\
\text { IBD. }\end{array}$ \\
\hline Comparison & $\begin{array}{l}\text { Comparison of patients similar to the intervention/exposure group, but without } \\
\text { IBD. }\end{array}$ & \\
\hline Outcome & $\begin{array}{l}\text { Same types of outcomes as in Table S1a. } \\
\text { Eligible outcomes include assessing periodontal disease outcomes before \& after } \\
\text { treatment for the IBD or IBD disease outcomes before \&, after treatment for the } \\
\text { periodontal disease }\end{array}$ & $\begin{array}{l}\text { No association of periodontal or IBD treatment with the } \\
\text { outcomes of the other disease. }\end{array}$ \\
\hline Study design & $\begin{array}{l}\text { Both randomized and non-randomized controlled human trials will be included } \\
\text { (Papageorgiou et al. 2014). If serious methodological discrepancies between the } \\
\text { two study designs are found (Papageorgiou et al., 2015), they will be assessed } \\
\text { separately. Any clinical setting will be included. }\end{array}$ & $\begin{array}{l}\text { Animal studies, clinical non-interventional and non-clinical } \\
\text { studies will be excluded. }\end{array}$ \\
\hline
\end{tabular}

IBD, inflammatory bowel disease; RCT, randomized controlled trial. 
Appendix S2. Search strategies used for each database with the corresponding results (last search Jan 2017).

\begin{tabular}{|c|c|c|c|c|}
\hline Electronic database & Search strategy used & Limits & $\begin{array}{l}\text { Hits up to } \\
\text { May } 2015\end{array}$ & $\begin{array}{l}\text { Update up } \\
\text { to Jan } 2017\end{array}$ \\
\hline \multicolumn{5}{|l|}{ General sources } \\
\hline $\begin{array}{l}\text { CDSR searched via Cochrane Library } \\
\text { www.thecochranelibrary.com }\end{array}$ & \multirow{3}{*}{$\begin{array}{l}\text { periodon* AND ("inflammatory bowel" OR "ulcerative colitis" OR } \\
\text { "Crohn's disease" OR "Crohn disease") }\end{array}$} & \multirow{3}{*}{ All text } & \multirow{3}{*}{1} & \multirow{3}{*}{1} \\
\hline $\begin{array}{l}\text { CENTRAL searched via Cochrane Library } \\
\text { www.thecochranelibrary.com }\end{array}$ & & & & \\
\hline $\begin{array}{l}\text { DARE searched via Cochrane Library } \\
\text { www.thecochranelibrary.com }\end{array}$ & & & & \\
\hline $\begin{array}{l}\text { MEDLINE (MeSH Terms) searched via PubMed } \\
\text { www.ncbi.nlm.nih.gov/sites/entrez/ }\end{array}$ & $\begin{array}{l}\text { periodon* AND ("inflammatory bowel" OR "ulcerative colitis" OR } \\
\text { "Crohn's disease" OR "Crohn disease") }\end{array}$ & Limit to humans & 91 & 16 \\
\hline $\begin{array}{l}\text { Scopus } \\
\text { www.scopus.com }\end{array}$ & $\begin{array}{l}\text { periodon* AND ("inflammatory bowel" OR "ulcerative colitis" OR } \\
\text { "Crohn's disease" OR "Crohn disease") }\end{array}$ & $\begin{array}{l}\text { Title/abstract/keywords } \\
\text { Limit to dentistry }\end{array}$ & 44 & 6 \\
\hline \multicolumn{5}{|l|}{ Open access sources } \\
\hline $\begin{array}{l}\text { DOAJ } \\
\text { http://www.doaj.org/ }\end{array}$ & $\begin{array}{l}\text {-"inflammatory bowel disease" AND periodon* } \\
\text {-Crohn AND periodon* } \\
\text {-"ulcerative colitis" AND periodon* }\end{array}$ & All fields & 6 & 0 \\
\hline \multicolumn{5}{|l|}{ Regional sources } \\
\hline $\begin{array}{l}\text { VHL } \\
\text { http://regional.bvsalud.org/php/index.php?lang=en }\end{array}$ & periodon* AND ("inflammatory bowel” OR “ulcerative colitis" OR Crohn) & Exclude MEDLINE and CENTRAL & 16 & 1 \\
\hline $\begin{array}{l}\text { Scielo } \\
\text { http://www.scielo.org/php/index.php?lang=en }\end{array}$ & ("inflammatory bowel" OR Crohn OR "ulcerative colitis") AND periodon* & - & 0 & 0 \\
\hline \multicolumn{5}{|l|}{ Grey literature sources } \\
\hline $\begin{array}{l}\text { Digital Dissertations searched via UMI/ProQuest } \\
\text { http://proquest.umi.com/login }\end{array}$ & $\begin{array}{l}\text { periodon* AND ("inflammatory bowel" OR "ulcerative colitis" OR } \\
\text { "Crohn's disease" OR "Crohn disease") }\end{array}$ & Limit to Dentistry & 54 & 8 \\
\hline $\begin{array}{l}\text { German National Library of Medicine (ZB MED) } \\
\text { http://www.medpilot.de }\end{array}$ & ("inflammatory bowel" OR Crohn OR "ulcerative colitis") AND periodon* & $\begin{array}{l}\text { Limit to Medizin/Gesundheit } \\
\text { Exclude MEDLINE, Books }\end{array}$ & 5 & 0 \\
\hline $\begin{array}{l}\text { ISI Web of Knowledge } \\
\text { http://apps.webofknowledge.com/ }\end{array}$ & $\begin{array}{l}\text { periodon* AND ("inflammatory bowel" OR "ulcerative colitis" OR } \\
\text { "Crohn's disease" OR "Crohn disease") }\end{array}$ & $\begin{array}{l}\text { Topic } \\
\text { Limit to Dentristry oral surgery } \\
\text { medicine }\end{array}$ & 112 & 0 \\
\hline $\begin{array}{l}\text { WHO trial search portal } \\
\text { http://apps.who.int/trialsearch/ }\end{array}$ & ("inflammatory bowel" OR Crohn OR "ulcerative colitis") AND periodon* & - & 0 & 0 \\
\hline $\begin{array}{l}\text { OpenSIGLE } \\
\text { http://www.opengrey.eu/ }\end{array}$ & ("inflammatory bowel" OR Crohn OR "ulcerative colitis") AND periodon* & - & 1 & 0 \\
\hline Overall articles from electronic search & & & 330 & 32 \\
\hline
\end{tabular}

\$ Highly-sensitive search strategy tested, in all databases, but included too few results and was omitted in many databases.

BBO, Bibliografia Brasileira de Odontologia; CDSR, Cochrane Database of Systematic Reviews; CENTRAL, Cochrane Central Register of Controlled Trials; DARE, Database of Abstracts of Reviews of Effects; DOAJ, Database of Open Access Journals; ISI, Institute for Scientific Information; LILACS, Literatura Latino-Americana e do Caribe em Ciências da Saúde; MeSH, Medical Subject Headings; Scielo, Scientific Eletronic Library on line; UMI, University Microfilms International; WHO, World Health Organization. 
Appendix S3. List of excluded/included studies.

\begin{tabular}{|c|c|c|}
\hline Nr. & Paper & \\
\hline 1 & $\begin{array}{l}\text { Acharya AAMDPSTKY. Developing an electronic dental record information model for } \\
\text { general dentistry [3416882]: University of Medicine and Dentistry of New Jersey; } 2010 .\end{array}$ & Excluded by title \\
\hline 2 & $\begin{array}{l}\text { Alasqah MAZDY. Salivary Biomarkers of Periodontal Disease in Smoker and Non-Smoker } \\
\text { Periodontitis Patients [1555895]: Tufts University School of Dental Medicine; } 2014 .\end{array}$ & Excluded by title \\
\hline 3 & $\begin{array}{l}\text { Albilia JATHY. Serum BMP-2, 4, } 7 \text { and AHSG in Patients with Heterotopic Ossification } \\
\text { Following Arthroplasty [Mr82350]: University of Toronto (Canada); } 2011 .\end{array}$ & Excluded by title \\
\hline 4 & $\begin{array}{l}\text { Bhatt AALAY. Mesenchymal stem cells from human gingiva ameliorate murine alimentary } \\
\text { mucositis [1496953]: University of Southern California; } 2011 .\end{array}$ & Excluded by title \\
\hline 5 & $\begin{array}{l}\text { Calhoun CCACRY. Cellular functions of cyclophilin A in actin stability and cyclosporine A } \\
\text { induced-gingival overgrowth [3356521]: University of California, Los Angeles; } 2008 .\end{array}$ & Excluded by title \\
\hline 6 & $\begin{array}{l}\text { Champaiboon CAHMCY. Calprotectin S100A9 structural domains regulate epithelial cell } \\
\text { resistance to bacterial invasion [3338934]: University of Minnesota; } 2008 \text {. }\end{array}$ & Excluded by title \\
\hline 7 & $\begin{array}{l}\text { Chino TACEAY. Regulation of inflammation and homeostatsis of oral mucosal tissue by } \\
\text { dendritic cells [3345634]: University of Washington; } 2008 \text {. }\end{array}$ & Excluded by title \\
\hline 8 & $\begin{array}{l}\text { Chou C-HADSRSMY. Association of Selected Candidate Gene Polymorphisms with } \\
\text { Periodontal Disease [3483535]: University of Medicine and Dentistry of New Jersey; } 2011 .\end{array}$ & Excluded by title \\
\hline 9 & $\begin{array}{l}\text { Divaris KAOAFY. Exploring the genetic basis of chronic periodontitis: A genome-wide } \\
\text { approach [3495471]: The University of North Carolina at Chapel Hill; } 2011 .\end{array}$ & Excluded by title \\
\hline 10 & $\begin{array}{l}\text { El-Awady AACCY. Porphyromonas gingivalis Escape-from Autophagy in Human Myeloid } \\
\text { Dendritic Cells via Minor Mfa-1 Fimbra-DC-Sign Interactions [3579936]: Georgia Regents } \\
\text { University; } 2014 .\end{array}$ & Excluded by title \\
\hline 11 & $\begin{array}{l}\text { Eskan MAY. Age-associated changes in innate immunity and their impacts on } \\
\text { inflammatory disease [3502286]: University of Louisville; } 2011 .\end{array}$ & Excluded by title \\
\hline 12 & $\begin{array}{l}\text { Fiedler LY. Role of decorin in control of endothelial cell behaviour [U584185]: Cardiff } \\
\text { University (United Kingdom); } 2007 .\end{array}$ & Excluded by title \\
\hline 13 & $\begin{array}{l}\text { Fleming PPABPHY. The effects of 2-DeNT oral topical powder on minor recurrent } \\
\text { aphthous ulcers [1539091]: The Texas A\&M University System Health Science Center; } \\
2013 .\end{array}$ & Excluded by title \\
\hline 14 & $\begin{array}{l}\text { Fredman GBAVDTEY. Resolvin E1 regulation of platelet functions: Novel pathways in } \\
\text { resolution [3357646]: Boston University; } 2009 \text {. }\end{array}$ & Excluded by title \\
\hline 15 & $\begin{array}{l}\text { Freire MAZHHY. Antibody mediated osseous regeneration [3513760]: University of } \\
\text { Southern California; } 2012 .\end{array}$ & Excluded by title \\
\hline 16 & $\begin{array}{l}\text { George JASDY. Cigarette smoke promotes genomic evolution in the periodontal pathogen } \\
\text { Porphyromonas gingivalis [1527649]: University of Louisville; } 2013 \text {. }\end{array}$ & Excluded by title \\
\hline 17 & $\begin{array}{l}\text { Ghadiri JAAOCCY. The prevalence of the uncultivated bacterium TM7a in human } \\
\text { subgingival plaque [1547092]: San Jose State University; } 2013 .\end{array}$ & Excluded by title \\
\hline 18 & $\begin{array}{l}\text { Hitimana HY. The Association between Functional Foods and Dental Caries Experience } \\
\text { [Mr67042]: McGill University (Canada); } 2009 .\end{array}$ & Excluded by title \\
\hline 19 & $\begin{array}{l}\text { Horst OVAD-CBAY. Defense mechanisms in human tooth pulp: Odontoblast functions } \\
\text { [3328407]: University of Washington; } 2008 \text {. }\end{array}$ & Excluded by title \\
\hline 20 & $\begin{array}{l}\text { Jaffray ECY. Characterisation of paediatric odontogenic bacteraemia [U591972]: } \\
\text { University of London, University College London (United Kingdom); 2006. }\end{array}$ & Excluded by title \\
\hline 21 & $\begin{array}{l}\text { Lenga YY. Osteopontin expression is required for myofibroblast differentiation [Mr44972]: } \\
\text { University of Toronto (Canada); } 2008 .\end{array}$ & Excluded by title \\
\hline 22 & $\begin{array}{l}\text { Loner CMY. Click scaffolds for the inhibition of Porphyromonas gingivalis and } \\
\text { Streptococcus gordonii biofilm formation [1521240]: University of Louisville; } 2012 .\end{array}$ & Excluded by title \\
\hline 23 & $\begin{array}{l}\text { Ma WAFSJY. Carbohydrate receptors, preterm labor, and periodontitis [3412216]: } \\
\text { University of California, San Francisco; } 2010 .\end{array}$ & Excluded by title \\
\hline 24 & $\begin{array}{l}\text { Maria OY. Identification and characterization of a cell source to regenerate salivary glands } \\
\text { [Nr72686]: McGill University (Canada); } 2010 .\end{array}$ & Excluded by title \\
\hline 25 & $\begin{array}{l}\text { McLean-Plunkett EAAMAEY. Vitamin D and Calcium Intake and the Prevalence of } \\
\text { Periodontal Disease in Postmenopausal Women [1519937]: State University of New York } \\
\text { at Buffalo; } 2012 .\end{array}$ & Excluded by title \\
\hline 26 & $\begin{array}{l}\text { Nibali LY. Analysis of genetic polymorphisms as risk factors for Aggressive Periodontitis } \\
\text { [U592181]: University of London, University College London (United Kingdom); } 2006 .\end{array}$ & Excluded by title \\
\hline 27 & Noueihed CY. An investigation of the effect of neighbourhood characteristics on traumatic & Excluded by title \\
\hline
\end{tabular}




\begin{tabular}{|c|c|c|}
\hline & $\begin{array}{l}\text { dental injuries among a sample of Quebec children [Mr56862]: McGill University } \\
\text { (Canada); } 2009 .\end{array}$ & \\
\hline 28 & $\begin{array}{l}\text { Odusanwo OAABOJY. Resolvin D1 prevents TNF-alpha-mediated disruption of salivary } \\
\text { epithelial formation [1500493]: State University of New York at Buffalo; } 2011 .\end{array}$ & Excluded by title \\
\hline 29 & $\begin{array}{l}\text { Oluwadara OOACFY. Molecular Biomarker Profiling and Translational Evidence-Based } \\
\text { Dentistry in Oral Lichen Planus and Oral Squamous Cell Carcinoma [3446847]: University } \\
\text { of California, Los Angeles; } 2010 .\end{array}$ & Excluded by title \\
\hline 30 & $\begin{array}{l}\text { Paes Batista da Silva AAOSY. Bacterial Characterization in Health and Periodontal } \\
\text { Diseases During Induced Gingival Inflammation [1513063]: The University of North } \\
\text { Carolina at Chapel Hill; } 2012 \text {. }\end{array}$ & Excluded by title \\
\hline 31 & $\begin{array}{l}\text { Paes Batista da Silva AY. Role of Osteopontin During Dextran Sulfate Sodium-induced } \\
\text { Colitis [Nr67676]: University of Toronto (Canada); } 2009 .\end{array}$ & Excluded by title \\
\hline 32 & $\begin{array}{l}\text { Papathanasiou EAGTTTCY. Levels of interferon-gamma (IFN-gamma), interleukin-4 (IL- } \\
\text { 4), interleukin-33 (IL-33) and thymic stromal lymphopoietin (TSLP) in gingival crevicular } \\
\text { fluid from patients with chronic periodontitis [1514563]: Tufts University School of Dental } \\
\text { Medicine; } 2012 \text {. }\end{array}$ & Excluded by title \\
\hline 33 & $\begin{array}{l}\text { Park MSMY. Oral health status in children undergoing treatment for neutropenia } \\
\text { [Mr76204]: University of Toronto (Canada); } 2011 .\end{array}$ & Excluded by title \\
\hline 34 & $\begin{array}{l}\text { Patil CSAKKLY. Targeting mRNA stability as a potential therapeutic for LPS-induced } \\
\text { inflammatory bone loss [3344637]: State University of New York at Buffalo; } 2007 .\end{array}$ & Excluded by title \\
\hline 35 & $\begin{array}{l}\text { Phattarataratip EABJAY. The role of salivary antimicrobial peptides in shaping } \\
\text { Streptococcus mutans ecology [3422183]: The University of lowa; } 2010 .\end{array}$ & Excluded by title \\
\hline 36 & $\begin{array}{l}\text { Phillips CRAKKBY. A qualitative survey of orthodontic extraction related patient } \\
\text { information available on the World Wide Web [1585315]: Saint Louis University; } 2014 .\end{array}$ & Excluded by title \\
\hline 37 & $\begin{array}{l}\text { Pongpichit BY. The prevalence and extent of school absences and factors related to } \\
\text { absences caused by dental conditions and dental care in Thai schoolchildren [U593118]: } \\
\text { University of London, University College London (United Kingdom); 2006. }\end{array}$ & Excluded by title \\
\hline 38 & $\begin{array}{l}\text { Prakasam SACCY. Modulating immune responses of Langerhans cells against microbial } \\
\text { associated molecular patterns [3401725]: State University of New York at Stony Brook; } \\
2009 \text {. }\end{array}$ & Excluded by title \\
\hline 39 & $\begin{array}{l}\text { Sahli MWAMAEY. The association between plasma 25-hydroxyvitamin D concentration } \\
\text { and oral bacteria among postmenopausal women [1519952]: State University of New } \\
\text { York at Buffalo; } 2012 \text {. }\end{array}$ & Excluded by title \\
\hline 40 & $\begin{array}{l}\text { Scheller ELAKPHY. Regulation of bone formation and pathology by local actions of leptin } \\
\text { in the bone marrow [3493053]: University of Michigan; } 2011 .\end{array}$ & Excluded by title \\
\hline 41 & $\begin{array}{l}\text { Schwarzberg KAKSTY. Oral polymicrobial communities and impact on human health } \\
\text { [3609026]: San Diego State University; } 2013 .\end{array}$ & Excluded by title \\
\hline 42 & $\begin{array}{l}\text { Sculley DVY. Inter-individual variation in saliva antioxidant status in relation to periodontal } \\
\text { disease [U191563]: University of Leicester (United Kingdom); } 2004 .\end{array}$ & Excluded by title \\
\hline 43 & $\begin{array}{l}\text { Seif MMAJS-JY. Etiopathology of autoimmune disease and dental perspectives } \\
\text { [1559614]: Boston University; } 2014 .\end{array}$ & Excluded by title \\
\hline 44 & $\begin{array}{l}\text { Seville LY. Study of tetracycline resistance determinants and their genetic supports in the } \\
\text { oral and faecal metagenomes of six European countries [U592369]: University of London, } \\
\text { University College London (United Kingdom); 2007. }\end{array}$ & Excluded by title \\
\hline 45 & $\begin{array}{l}\text { Shapiro JLAPMLY. The endocytosis of enamel matrix protein derivatives [3368646]: } \\
\text { University of Southern California; } 2009 \text {. }\end{array}$ & Excluded by title \\
\hline 46 & $\begin{array}{l}\text { Shrestha DAYPY. Implementing Teledentistry in Nepal: Using Mobile Phone and Internet } \\
\text { as Store-and-Forward Method [1507181]: University of California, Davis; } 2011 .\end{array}$ & Excluded by title \\
\hline 47 & $\begin{array}{l}\text { Singer JAAJSY. Vitamin D and chronic pain: A comprehensive review [1545731]: Temple } \\
\text { University; } 2013 .\end{array}$ & Excluded by title \\
\hline 48 & $\begin{array}{l}\text { Villoria PAHVY. A clinical study to assess effect of oral hygiene with a probiotic organism } \\
\text { on dental plaque pH and on plaque bacteria including Streptococcus mutans [1546977]: } \\
\text { State University of New York at Buffalo; } 2013 \text {. }\end{array}$ & Excluded by title \\
\hline 49 & $\begin{array}{l}\text { Yezdani GAZPY. Role of VDR in host immune response to Porphyromonas gingivalis } \\
\text { infection [1499774]: The University of Alabama at Birmingham; } 2011 .\end{array}$ & Excluded by title \\
\hline 50 & $\begin{array}{l}\text { Zhang GARJDY. Streptococcus cristatus modulates epithelial innate immune response } \\
\text { through regulating nuclear factor-kappa B pathway [3389387]: University of Minnesota; } \\
2009 \text {. }\end{array}$ & Excluded by title \\
\hline 51 & Zhang SAOSY. Regulation of Inflammatory Genes Involved in Periodontal Diseases by & Excluded by title \\
\hline
\end{tabular}


DNA Methylation [3464948]: The University of North Carolina at Chapel Hill; 2011.

Zhang SAOSY. Expression of interleukin-37 (IL37) and the genetic variations of IL37 in

52 relation to chronic periodontitis [1557234]: The University of North Carolina at Chapel Hill; 2014.

Abo T, Kawamura T, Kawamura H, Tomiyama-Miyaji C, Kanda Y. Relationship between

53 diseases accompanied by tissue destruction and granulocytes with surface adrenergic receptors. Immunologic research. 2007;37(3):201-10. Epub 2007/09/18.

Ayangco L, Rogers RS, 3rd, Sheridan PJ. Pyostomatitis vegetans as an early sign of

54 reactivation of Crohn's disease: a case report. J Periodontol. 2002;73(12):1512-6. Epub 2003/01/28.

Barksby HE, Lea SR, Preshaw PM, Taylor JJ. The expanding family of interleukin-1

55 cytokines and their role in destructive inflammatory disorders. Clinical and experimental immunology. 2007;149(2):217-25.

Castellanos JL. [Periodontal immunopathology]. Revista ADM : organo oficial de la

56 Asociacion Dental Mexicana. 1985;42(5):149-54. Epub 1985/09/01. Immunopatologia parodontal.

57 Chi AC, Neville BW, Krayer JW, Gonsalves WC. Oral manifestations of systemic disease. American family physician. 2010;82(11):1381-8.

Excluded by title

Excluded by title

Diegelmann J, Czamara D, Le Bras E, Zimmermann E, Olszak T, Bedynek A, et al.

58 Intestinal DMBT1 expression is modulated by Crohn's disease-associated IL23R variants and by a DMBT1 variant which influences binding of the transcription factors CREB1 and ATF-2. PloS one. 2013;8(11):e77773.

59 Duran-Pinedo AE, Paster B, Teles R, Frias-Lopez J. Correlation network analysis applied to complex biofilm communities. PloS one. 2011;6(12):e28438. Epub 2011/12/14.

60 Elias MK, Mateen FJ, Weiler CR. The Melkersson-Rosenthal syndrome: a retrospective study of biopsied cases. Journal of neurology. 2013;260(1):138-43. Epub 2012/07/28.

Folwaczny M, Glas J, Torok HP, Limbersky O, Folwaczny C. Toll-like receptor (TLR) 2

61 and 4 mutations in periodontal disease. Clinical and experimental immunology. 2004;135(2):330-5. Epub 2004/01/24.

Fry L, Baker BS, Powles AV, Fahlen A, Engstrand L. Is chronic plaque psoriasis triggered

62 by microbiota in the skin? The British journal of dermatology. 2013;169(1):47-52. Epub 2013/03/26.

63 Garrido-Mesa N, Zarzuelo A, Galvez J. Minocycline: far beyond an antibiotic. British journal of pharmacology. 2013;169(2):337-52. Epub 2013/02/28.

$64 \quad$ Glade MJ. Vitamin D: health panacea or false prophet? Nutrition (Burbank, Los Angeles County, Calif). 2013;29(1):37-41. Epub 2012/10/23.

65 Hajishengallis G, Darveau RP, Curtis MA. The keystone-pathogen hypothesis. Nature reviews Microbiology. 2012;10(10):717-25. Epub 2012/09/04.

Healy CM, Farthing PM, Williams DM, Thornhill MH. Pyostomatitis vegetans and

66 associated systemic disease. A review and two case reports. Oral surgery, oral medicine, and oral pathology. 1994;78(3):323-8.

67 Hofstad T. Immune responses to anaerobic gram-negative bacteria in health and disease. Die Nahrung. 1984;28(6-7):717-21. Epub 1984/01/01.

Ichiishi S, Tanaka K, Nakao K, Izumi K, Mikamo H, Watanabe K. First isolation of

68 Desulfovibrio from the human vaginal flora. Anaerobe. 2010;16(3):229-33. Epub 2010/02/18.

$69 \quad$ Ingram C. Melkersson-Rosenthal syndrome or oro-facial granulomatosis (OFG): an update. Journal of the New Zealand Society of Periodontology. 1999(84):24-5. Epub 2000/05/24.

Jorth P, Turner KH, Gumus P, Nizam N, Buduneli N, Whiteley M. Metatranscriptomics of

70 the human oral microbiome during health and disease. mBio. 2014;5(2):e01012-14. Epub 2014/04/03.

71 Kaakoush NO, Mitchell HM. Campylobacter concisus - A new player in intestinal disease. Frontiers in cellular and infection microbiology. 2012;2:4. Epub 2012/08/25.

Excluded by title

Excluded by title

Excluded by title

Excluded by title

Excluded by title

Excluded by title

Excluded by title

Excluded by title

Excluded by title

Excluded by title

Excluded by title

Excluded by title

Excluded by title

Excluded by title

Excluded by title

Excluded by title

Excluded by title

Excluded by title

Kuehbacher T, Rehman A, Lepage P, Hellmig S, Folsch UR, Schreiber S, et al. Intestinal

72 TM7 bacterial phylogenies in active inflammatory bowel disease. Journal of medical microbiology. 2008;57(Pt 12):1569-76.

73 Kverka M, Tlaskalova-Hogenova $\mathrm{H}$. Two faces of microbiota in inflammatory and autoimmune diseases: triggers and drugs. APMIS : acta pathologica, microbiologica, et

Excluded by title

Excluded by title 
immunologica Scandinavica. 2013;121(5):403-21.

Ladero JM. Influence of polymorphic $\mathrm{N}$-acetyltransferases on non-malignant spontaneous disorders and on response to drugs. Current drug metabolism. 2008;9(6):532-7. Epub 2008/08/06.

Lakschevitz FS, Glogauer M. High-purity neutrophil isolation from human peripheral blood and saliva for transcriptome analysis. Methods in molecular biology (Clifton, $\mathrm{NJ}$ ). 2014;1124:469-83. Epub 2014/02/08.

Lazaro P. [Oral rehabilitation of a patient with Crohn's disease]. Le Chirurgien-dentiste de

76 France. 1990;60(544-545):57-64. Epub 1990/12/20. Rehabilitation buccale chez un sujet atteint de maladie de Crohn.

77 Lodowska J, Wolny D, Jaworska-Kik M, Kurkiewicz S, Dzierzewicz Z, Weglarz L. The

77 chemical composition of endotoxin isolated from intestinal strain of Desulfovibrio desulfuricans. TheScientificWorldJournal. 2012;2012:647352. Epub 2012/05/26.

Loos BG, Fiebig A, Nothnagel M, Jepsen S, Groessner-Schreiber B, Franke A, et al.

$78 \quad$ NOD1 gene polymorphisms in relation to aggressive periodontitis. Innate immunity. 2009;15(4):225-32. Epub 2009/07/10.

79 Nibali L, Donos N, Henderson B. Periodontal infectogenomics. Journal of medical microbiology. 2009;58(Pt 10):1269-74. Epub 2009/06/17.

Excluded by title

Excluded by title

Excluded by title Ogawa T, Tarkowski A, McGhee ML, Moldoveanu Z, Mestecky J, Hirsch HZ, et al.

80 Analysis of human IgG and IgA subclass antibody-secreting cells from localized chronic inflammatory tissue. Journal of immunology (Baltimore, Md : 1950). 1989;142(4):1150-8. Epub 1989/02/15.

Pagoldh M, Lange S, Jennische E, Almer S, Bostrom EA, Eriksson A. Faecal analysis and

81 plasma complement factor $3 c$ levels at admission for an acute attack of ulcerative colitis are predictive of the need for colectomy. European journal of gastroenterology \& hepatology. 2014;26(3):295-300. Epub 2014/01/15.

82 Redlich K, Smolen JS. Inflammatory bone loss: pathogenesis and therapeutic intervention. Nature reviews Drug discovery. 2012;11(3):234-50. Epub 2012/03/02.

Rees TD. Orofacial granulomatosis and related conditions. Periodontol 2000. 1999;21:145-57. Epub 1999/11/07.

Reismann P, Racz K, Tulassay Z. [Polymorphisms of the Toll-like receptor 4 gene and their potential role in infectious diseases and chronic inflammatory disorders]. Orvosi hetilap. 2008;149(38):1791-9. Epub 2008/09/23. A Toll-like receptor-4genpolimorfizmusok, valamint lehetseges klinikai szerepuk fertozesekben es kronikus gyulladasos betegsegekben.

Rezaie A, Ghorbani F, Eshghtork A, Zamani MJ, Dehghan G, Taghavi B, et al. Alterations in salivary antioxidants, nitric oxide, and transforming growth factor-beta 1 in relation to disease activity in Crohn's disease patients. Annals of the New York Academy of Sciences. 2006;1091:110-22. Epub 2007/03/08.

Rogers RS, 3rd, Movius DL, Pierre RV. Lymphocyte-epithelial cell interactions in oral

86 mucosal inflammatory diseases. The Journal of investigative dermatology. 1976;67(5):599-602. Epub 1976/11/01.

Sadler E, Klausegger A, Muss W, Deinsberger U, Pohla-Gubo G, Laimer M, et al. Novel

$87 \quad$ KIND1 gene mutation in Kindler syndrome with severe gastrointestinal tract involvement. Archives of dermatology. 2006;142(12):1619-24. Epub 2006/12/21.

Sanz M, van Winkelhoff AJ. Periodontal infections: understanding the complexity--

88 consensus of the Seventh European Workshop on Periodontology. J Clin Periodontol. 2011;38 Suppl 11:3-6. Epub 2011/03/01.

Schwalfenberg GK. A review of the critical role of vitamin $D$ in the functioning of the

89 immune system and the clinical implications of vitamin D deficiency. Molecular nutrition \& food research. 2011;55(1):96-108.

Scott DA, Martin M. Exploitation of the nicotinic anti-inflammatory pathway for the

90 treatment of epithelial inflammatory diseases. World journal of gastroenterology : WJG. 2006;12(46):7451-9. Epub 2006/12/15.

Singh VP, Sharma J, Babu S, Rizwanulla, Singla A. Role of probiotics in health and

91 disease: a review. JPMA The Journal of the Pakistan Medical Association. 2013;63(2):253-7. Epub 2013/07/31. patient. The Journal of oral implantology. 1990;16(3):211-7. Epub 1990/01/01.

Excluded by title

Excluded by title

Excluded by title

Excluded by title

Excluded by title

Excluded by title

Excluded by title

Excluded by title

Excluded by title

Excluded by title

Excluded by title

Excluded by title

Excluded by title

Excluded by title

Excluded by title

Excluded by title 


\begin{tabular}{|c|c|c|}
\hline 93 & $\begin{array}{l}\text { Taichman NS, Wilton JM. Leukotoxicity of an extract from Actinobacillus } \\
\text { actinomycetemcomitans for human gingival polymorphonuclear leukocytes. Inflammation. } \\
1981 ; 5(1): 1-12 \text {. Epub 1981/03/01. }\end{array}$ & Excluded by title \\
\hline 94 & $\begin{array}{l}\text { Tiedemann C, Wetzel A. [Periodontitis as manifestation of a systemic disease. A case } \\
\text { report]. Schweizer Monatsschrift fur Zahnmedizin = Revue mensuelle suisse d'odonto- } \\
\text { stomatologie = Rivista mensile svizzera di odontologia e stomatologia / SSO. } \\
2001 ; 111(9): 1091-102 . \text { Epub 2001/10/26. Parodontitis als Manifestation einer } \\
\text { Systemerkrankung. Eine Fallprasentation. }\end{array}$ & Excluded by title \\
\hline 95 & $\begin{array}{l}\text { Tlaskalova-Hogenova H, Stepankova R, Hudcovic T, Tuckova L, Cukrowska B, Lodinova- } \\
\text { Zadnikova R, et al. Commensal bacteria (normal microflora), mucosal immunity and } \\
\text { chronic inflammatory and autoimmune diseases. Immunology letters. 2004;93(2-3):97- } \\
\text { 108. Epub 2004/05/26. }\end{array}$ & Excluded by title \\
\hline 96 & $\begin{array}{l}\text { van der Bijl P, Broeksma J. Acute bronchospasm following administration of lidocaine. } \\
\text { Anesthesia \& pain control in dentistry. 1993;2(4):203-5. Epub 1993/01/01. }\end{array}$ & Excluded by title \\
\hline 97 & $\begin{array}{l}\text { Wei B, Dalwadi H, Gordon LK, Landers C, Bruckner D, Targan SR, et al. Molecular } \\
\text { cloning of a Bacteroides caccae TonB-linked outer membrane protein identified by an } \\
\text { inflammatory bowel disease marker antibody. Infection and immunity. 2001;69(10):6044- } \\
\text { 54. Epub 2001/09/13. }\end{array}$ & Excluded by title \\
\hline 98 & $\begin{array}{l}\text { Yeoh N, Burton JP, Suppiah P, Reid G, Stebbings S. The role of the microbiome in } \\
\text { rheumatic diseases. Current rheumatology reports. 2013;15(3):314. Epub 2013/02/05. }\end{array}$ & Excluded by title \\
\hline 99 & $\begin{array}{l}\text { Aarthi JJ, Darendeliler MA, Pushparaj PN. Dissecting the role of the S1P/S1PR axis in } \\
\text { health and disease. Journal of Dental Research. } 2011 ; 90(7): 841-54 .\end{array}$ & Excluded by title \\
\hline 100 & $\begin{array}{l}\text { Alsaadi G, Quirynen M, Komárek A, Van Steenberghe D. Impact of local and systemic } \\
\text { factors on the incidence of oral implant failures, up to abutment connection. Journal of } \\
\text { Clinical Periodontology. 2007;34(7):610-7. }\end{array}$ & Excluded by title \\
\hline 101 & $\begin{array}{l}\text { Alsaadi G, Quirynen M, Komárek A, Van Steenberghe D. Impact of local and systemic } \\
\text { factors on the incidence of late oral implant loss. Clinical Oral Implants Research. } \\
2008 ; 19(7): 670-6 .\end{array}$ & Excluded by title \\
\hline 102 & $\begin{array}{l}\text { Alsaadi G, Quirynen M, Michiles K, Teughels W, Komárek A, Van Steenberghe D. Impact } \\
\text { of local and systemic factors on the incidence of failures up to abutment connection with } \\
\text { modified surface oral implants. Journal of Clinical Periodontology. 2008;35(1):51-7. }\end{array}$ & Excluded by title \\
\hline 103 & $\begin{array}{l}\text { Balshi TJ, Wolfinger GJ. Management of the posterior maxilla in the compromised patient: } \\
\text { Historical, current, and future perspectives. Periodontology 2000. 2003;33:67-81. }\end{array}$ & Excluded by title \\
\hline 104 & $\begin{array}{l}\text { Dawson DR, Branch-Mays G, Gonzalez OA, Ebersole JL. Dietary modulation of the } \\
\text { inflammatory cascade. Periodontology } 2000.2014 ; 64(1): 161-97 .\end{array}$ & Excluded by title \\
\hline 105 & $\begin{array}{l}\text { Folwaczny M, Glas J, Török HP, Mauermann D, Folwaczny C. The 3020insC mutation of } \\
\text { the NOD2/CARD15 gene in patients with periodontal disease. European Journal of Oral } \\
\text { Sciences. 2004;112(4):316-9. }\end{array}$ & Excluded by title \\
\hline 106 & $\begin{array}{l}\text { Johannsen A, Susin C, Gustafsson A. Smoking and inflammation: Evidence for a } \\
\text { synergistic role in chronic disease. Periodontology } 2000.2014 ; 64(1): 111-26 .\end{array}$ & Excluded by title \\
\hline 107 & $\begin{array}{l}\text { Kebschull M, Demmer R, Behle JH, Pollreisz A, Heidemann J, Belusko PB, et al. } \\
\text { Granulocyte chemotactic protein } 2 \text { (gcp-2/cxcl6) complements interleukin-8 in periodontal } \\
\text { disease. Journal of Periodontal Research. 2009;44(4):465-71. }\end{array}$ & Excluded by title \\
\hline 108 & $\begin{array}{l}\text { Laine ML, Murillo LS, Morré SA, Winkel EG, Peña AS, Van Winkelhoff AJ. CARD15 gene } \\
\text { mutations in periodontitis. Journal of Clinical Periodontology. 2004;31(10):890-3. }\end{array}$ & Excluded by title \\
\hline 109 & $\begin{array}{l}\text { Li Z, Jiang CM, An S, Cheng Q, Huang YF, Wang YT, et al. Immunomodulatory properties } \\
\text { of dental tissue-derived mesenchymal stem cells. Oral Diseases. 2014;20(1):25-34. }\end{array}$ & Excluded by title \\
\hline 110 & $\begin{array}{l}\text { Nagpal S, Acharya AB, Thakur SL. Periodontal disease and anemias associated with } \\
\text { Crohn's disease. A case report. The New York state dental journal. 2012;78(2):47-50. }\end{array}$ & Excluded by title \\
\hline 111 & $\begin{array}{l}\text { Noack B, Görgens H, Hoffmann T, Schackert HK. CARD15 gene variants in aggressive } \\
\text { periodontitis. Journal of Clinical Periodontology. 2006;33(11):779-83. }\end{array}$ & Excluded by title \\
\hline 112 & $\begin{array}{l}\text { Ouhara K, Kawai T, Silva MJ, Fujita T, Hayashida K, Karimbux NY, et al. Expression } \\
\text { levels of novel cytokine IL-32 in periodontitis and its role in the suppression of IL-8 } \\
\text { production by human gingival fibroblasts stimulated with porphyromonas gingivalis. } \\
\text { Journal of Oral Microbiology. 2012;4(2012). }\end{array}$ & Excluded by title \\
\hline 113 & $\begin{array}{l}\text { Oz HS, Ebersole JL. A novel murine model for chronic inflammatory alveolar bone loss. } \\
\text { Journal of Periodontal Research. 2010;45(1):94-9. }\end{array}$ & Excluded by title \\
\hline 114 & $\begin{array}{l}\text { Palma-Carrió C, Maestre-Ferrín L, Peñarrocha-Oltra D, Peñarrocha-Diago MA, } \\
\text { Peñarrocha-Diago M. Risk factors associated with early failure of dental implants. A }\end{array}$ & Excluded by title \\
\hline
\end{tabular}


literature review. Medicina Oral, Patologia Oral y Cirugia Bucal. 2011;16(4):e514-e7.

Paquette DW, Rosenberg A, Lohinai Z, Southan GJ, Williams RC, Offenbacher S, et al. Inhibition of experimental gingivitis in beagle dogs with topical mercaptoalkylguanidines. Journal of Periodontology. 2006;77(3):385-91.

Pietropaoli D, Del Pinto R, Corridoni D, Rodriguez-Palacios A, Di Stefano G, Monaco A, et

116 al. Occurrence of spontaneous periodontal disease in the SAMP1/YitFc murine model of crohn disease. Journal of Periodontology. 2014;85(12):1799-805.

Schaefer AS, Jochens A, Dommisch H, Graetz C, Jockel-Schneider Y, Harks I, et al. A

117 large candidate-gene association study suggests genetic variants at IRF5 and PRDM1 to be associated with aggressive periodontitis. Journal of Clinical Periodontology.

2014;41(12):1122-31.

118 Sigusch BW. Periodontitis as manifestation of Crohn's disease in primary dentition: A case report. Journal of Dentistry for Children. 2004;71(3):193-6.

Van Steenberghe D, Jacobs R, Desnyder M, Maffei G, Quirynen M. The relative impact of

119 local and endogenous patient-related factors on implant failure up to the abutment stage. Clinical Oral Implants Research. 2002;13(6):617-22.

Wiernicka-Menkiszak M, Dembowska E, Samulak-Zielińska R. Localized aggressive

120 periodontitis - Diagnostics, epidemiology, etiopathogenesis. Dental and Medical Problems. 2012;49(4):567-75.

121 AAP. Periodontal disease as a potential risk factor for systemic diseases. Journal of Periodontology. 1998;69(7):841-50.

Al-Rasheed A, Scheerens H, Srivastava AK, Rennick DM, Tatakis DN. Accelerated

122 alveolar bone loss in mice lacking interleukin-10: late onset. Journal of Periodontal Research. 2004;39(3):194-8.

Brandtzaeg P. Inflammatory bowel disease: clinics and pathology - Do inflammatory

123 bowel disease and periodontal disease have similar immunopathogeneses? Acta Odontologica Scandinavica. 2001;59(4):235-43.

Breivik T, Gundersen Y, Gjermo P, Taylor SM, Woodruff TM, Opstad PK. Oral treatment

124 with complement factor C5a receptor (CD88) antagonists inhibits experimental periodontitis in rats. Journal of Periodontal Research. 2011;46(6):643-7.

125 Cotti E, Schirru E, Acquas E, Usai P. An Overview on Biologic Medications and Their Possible Role in Apical Periodontitis. Journal of Endodontics. 2014;40(12):1902-11.

Excluded by title

Excluded by title

Excluded by title

Excluded by title

Excluded by title

Excluded by title

Excluded by title

Excluded by title

Excluded by title

Cutler CW, Teng Y-TA. Oral mucosal dendritic cells and periodontitis: many sides of the same coin with new twists. Periodontology 2000. 2007;45:35-50.

Dabelsteen S, Wandall HH, Gron B, Dabelsteen E. Keratinocyte growth factor mRNA expression in periodontal ligament fibroblasts. European Journal of Oral Sciences. 1997;105(6):593-8.

Dezerega A, Pozo P, Hernandez M, Oyarzun A, Rivera O, Dutzan N, et al. Chemokine

128 Monocyte Chemoattractant Protein-3 in Progressive Periodontal Lesions in Patients With Chronic Periodontitis. Journal of Periodontology. 2010;81(2):267-76.

129 Folwaczny M, Tengler B, Glas J. Variants of the human NR112 (PXR) locus in chronic periodontitis. Journal of Periodontal Research. 2012;47(2):174-9.

Fredriksson M, Bergstrom K, Asman B. IL-8 and TNF-alpha from peripheral neutrophils

130 and acute-phase proteins in periodontitis - Effect of cigarette smoking: a pilot study. Journal of Clinical Periodontology. 2002;29(2):123-8.

1 Gemmell E, Marshall RI, Seymour GJ. Cytokines and prostaglandins in immune

131 homeostasis and tissue destruction in periodontal disease. Periodontology 2000. 1997;14:112-43.

132 Glimvall $\mathrm{P}$, Wickstrom C, Jansson $\mathrm{H}$. Elevated levels of salivary lactoferrin, a marker for chronic periodontitis? Journal of Periodontal Research. 2012;47(5):655-60.

Excluded by title

Excluded by title

Excluded by title

Excluded by title Guimaraes MR, Coimbra LS, de Aquino SG, Spolidorio LC, Kirkwood KL, Rossa C, Jr.

133 Potent anti-inflammatory effects of systemically administered curcumin modulate periodontal disease in vivo. Journal of Periodontal Research. 2011;46(2):269-79.

Hodge PJ, Riggio MP, Kinane DF. Failure to detect an association with IL1 genotypes in

134 European Caucasians with generalised early onset periodontitis. Journal of Clinical Periodontology. 2001;28(5):430-6.

Johnson RB, Serio FG. Interleukin-18 concentrations and the pathogenesis of periodontal disease. Journal of Periodontology. 2005;76(5):785-90.

136 Kasprzak A, Surdacka A, Tomczak M, Konkol M. Role of high endothelial postcapillary

Excluded by title

Excluded by title

Excluded by title

Excluded by title

Excluded by title

Excluded by title

Excluded by title

Excluded by title

Excluded by title 


\begin{tabular}{|c|c|c|}
\hline & $\begin{array}{l}\text { venules and selected adhesion molecules in periodontal diseases: a review. Journal of } \\
\text { Periodontal Research. 2013;48(1):1-21. }\end{array}$ & \\
\hline 137 & $\begin{array}{l}\text { Laine ML, Farre MA, Crusius JBA, van Winkelhoff AJ, Pena AS. The mouthwash: A non- } \\
\text { invasive sampling method to study cytokine gene polymorphisms. Journal of } \\
\text { Periodontology. } 2000 ; 71(8): 1315-8 \text {. }\end{array}$ & Excluded by title \\
\hline 138 & $\begin{array}{l}\text { Laine ML, Leonhardt A, Roos-Jansaker A-M, Salvador Pena A, van Winkelhoff AJ, Winkel } \\
\text { EG, et al. IL-1RN gene polymorphism is associated with peri-implantitis. Clinical Oral } \\
\text { Implants Research. 2006;17(4):380-5. }\end{array}$ & Excluded by title \\
\hline 139 & $\begin{array}{l}\text { Li M, Firth JD, Putnins EE. Keratinocyte growth factor-1 expression in healthy and } \\
\text { diseased human periodontal tissues. Journal of Periodontal Research. 2005;40(2):118-28. }\end{array}$ & Excluded by title \\
\hline 140 & $\begin{array}{l}\text { Loos BG, John RP, Laine ML. Identification of genetic risk factors for periodontitis and } \\
\text { possible mechanisms of action. Journal of Clinical Periodontology. 2005;32:159-79. }\end{array}$ & Excluded by title \\
\hline 141 & $\begin{array}{l}\text { Loos BG, Tjoa S. Host-derived diagnostic markers for periodontitis: do they exist in } \\
\text { gingival crevice fluid? Periodontology } 2000.2005 ; 39: 53-72 \text {. }\end{array}$ & Excluded by title \\
\hline 142 & $\begin{array}{l}\text { Loubinoux J, Bisson-Boutelliez C, Miller N, Le Faou AE. Isolation of the provisionally } \\
\text { named Desulfovibrio fairfieldensis from human periodontal pockets. Oral Microbiology and } \\
\text { Immunology. 2002;17(5):321-3. }\end{array}$ & Excluded by title \\
\hline 143 & $\begin{array}{l}\text { May NY, Tatakis DN. Accelerated alveolar bone loss in male HLA-B27 transgenic rats: } \\
\text { adult onset. Journal of Periodontal Research. } 2004 ; 39(1): 33-6 \text {. }\end{array}$ & Excluded by title \\
\hline 144 & $\begin{array}{l}\text { Messora MR, Oliveira LFF, Foureaux RC, Taba M, Jr., Zangeronimo MG, Furlaneto FAC, } \\
\text { et al. Probiotic Therapy Reduces Periodontal Tissue Destruction and Improves the } \\
\text { Intestinal Morphology in Rats With Ligature-Induced Periodontitis. Journal of } \\
\text { Periodontology. 2013;84(12):1818-26. }\end{array}$ & Excluded by title \\
\hline 145 & $\begin{array}{l}\text { Morton RS, Dongari-Bagtzoglou Al. Cyclooxygenase-2 is upregulated in inflamed gingival } \\
\text { tissues. Journal of Periodontology. } 2001 ; 72(4): 461-9 \text {. }\end{array}$ & Excluded by title \\
\hline 146 & $\begin{array}{l}\text { Park J-C, Kim B-K, Jung I-H, Choi E, Kim C-S. Alveolar Bone Resorption Induced by } \\
\text { CD4(+)CD45RB High-Density T-Cell Transfer in Immunocompromised Mice. Journal of } \\
\text { Periodontology. 2014;85(9):E339-E47. }\end{array}$ & Excluded by title \\
\hline 147 & $\begin{array}{l}\text { Sakellari D, Koukoudetsos S, Arsenakis M, Konstantinidis A. Prevalence of IL-1A and IL- } \\
\text { 1B polymorphisms in a Greek population. Journal of Clinical Periodontology. } \\
2003 ; 30(1): 35-41 \text {. }\end{array}$ & Excluded by title \\
\hline 148 & $\begin{array}{l}\text { Sanaie AR, Firth JD, Uitto VJ, Putnins EE. Keratinocyte growth factor (KGF)-1 and-2 } \\
\text { protein and gene expression in human gingival fibroblasts. Journal of Periodontal } \\
\text { Research. 2002;37(1):66-74. }\end{array}$ & Excluded by title \\
\hline 149 & $\begin{array}{l}\text { Scapoli C, Mamolini E, Trombelli L. Role of IL-6, TNF-A and LT-A variants in the } \\
\text { modulation of the clinical expression of plaque-induced gingivitis. Journal of Clinical } \\
\text { Periodontology. 2007;34(12):1031-8. }\end{array}$ & Excluded by title \\
\hline 150 & $\begin{array}{l}\text { Schaefer AS, Bochenek G, Manke T, Nothnagel M, Graetz C, Thien A, et al. Validation of } \\
\text { reported genetic risk factors for periodontitis in a large-scale replication study. Journal of } \\
\text { Clinical Periodontology. } 2013 ; 40(6): 563-72 \text {. }\end{array}$ & Excluded by title \\
\hline 151 & $\begin{array}{l}\text { Stamatova I, Meurman JH. Probiotics and periodontal disease. Periodontology } 2000 . \\
\text { 2009;51:141-51. }\end{array}$ & Excluded by title \\
\hline 152 & $\begin{array}{l}\text { Sugerman PB, Savage NW, Seymour GJ. Clonal Expansion of Lymphocytes from Oral } \\
\text { Lichen-Planus Lesions. Journal of Oral Pathology \& Medicine. 1993;22(3):126-31. }\end{array}$ & Excluded by title \\
\hline 153 & $\begin{array}{l}\text { Takahashi I, Fujihashi K, Kiyono H. Mucosal regulatory cells in the gastrointestinal tract } \\
\text { and periodontium. Periodontology 2000. 2010;54:247-56. }\end{array}$ & Excluded by title \\
\hline 154 & $\begin{array}{l}\text { Trombelli L, Scapoli C, Tatakis DN, Grassi L. Modulation of clinical expression of plaque- } \\
\text { induced gingivitis: effects of personality traits, social support and stress. Journal of Clinical } \\
\text { Periodontology. } 2005 ; 32(11): 1143-50 \text {. }\end{array}$ & Excluded by title \\
\hline 155 & $\begin{array}{l}\text { Vuotto C, Longo F, Donelli G. Probiotics to counteract biofilm-associated infections: } \\
\text { promising and conflicting data. International Journal of Oral Science. } 2014 ; 6(4): 189-94 .\end{array}$ & Excluded by title \\
\hline 156 & $\begin{array}{l}\text { Wang D, Nagasawa T, Chen Y, Ushida Y, Kobayashi H, Takeuchi Y, et al. Molecular } \\
\text { mimicry of Aggregatibacter actinomycetemcomitans with beta } 2 \text { glycoprotein I. Oral } \\
\text { Microbiology and Immunology. 2008;23(5):401-5. }\end{array}$ & Excluded by title \\
\hline 157 & $\begin{array}{l}\text { Markovic L, Besir Ljuskovic. Oral manifestations of Crohn's disease. Srpsko lekarsko } \\
\text { društvo 2004;51:0039-1743. }\end{array}$ & Excluded by abstract \\
\hline 158 & $\begin{array}{l}\text { Brito F, Zaltman C, Carvalho AT, Fischer RG, Persson R, Gustafsson A, et al. Subgingival } \\
\text { microflora in inflammatory bowel disease patients with untreated periodontitis. European } \\
\text { journal of gastroenterology \& hepatology. } 2013 ; 25(2): 239-45 \text {. Epub } 2012 / 10 / 13 \text {. }\end{array}$ & Excluded by abstract \\
\hline
\end{tabular}




\begin{tabular}{|c|c|c|}
\hline 159 & $\begin{array}{l}\text { Colella G, Riegler G, Lanza A, Tartaro GP, Russo Ml, Tartaglione M. [Changes in the } \\
\text { mouth mucosa in patients with chronic inflammatory intestinal diseases]. Minerva } \\
\text { stomatologica. 1999;48(9):367-71. Epub 2000/01/19. Alterazioni della mucosa orale in } \\
\text { pazienti affetti da malattie infiammatorie croniche intestinali. }\end{array}$ & Excluded by abstract \\
\hline 160 & $\begin{array}{l}\text { Gargiulo AV, Ladone JA, Toto PD, Logiudice J. Crohn's disease: early detection by } \\
\text { gingival biopsy. Periodontal case reports : a publication of the Northeastern Society of } \\
\text { Periodontists. 1989;11(1):20-2. Epub 1989/01/01. }\end{array}$ & Excluded by abstract \\
\hline 161 & $\begin{array}{l}\text { Groselj D, Grabec I, Seme K, Ihan A, Ferkolj I. Prediction of clinical response to anti-TNF } \\
\text { treatment by oral parameters in Crohn's disease. Hepato-gastroenterology. } \\
2008 ; 55(81): 112-9 \text {. Epub 2008/05/30. }\end{array}$ & Excluded by abstract \\
\hline 162 & $\begin{array}{l}\text { Han YW, Wang X. Mobile microbiome: oral bacteria in extra-oral infections and } \\
\text { inflammation. J Dent Res. 2013;92(6):485-91. Epub 2013/04/30. }\end{array}$ & Excluded by abstract \\
\hline 163 & $\begin{array}{l}\text { Kido J, Kido R, Suryono, Kataoka M, Fagerhol MK, Nagata T. Calprotectin release from } \\
\text { human neutrophils is induced by Porphyromonas gingivalis lipopolysaccharide via the CD- } \\
\text { 14-Toll-like receptor-nuclear factor kappaB pathway. J Periodontal Res. 2003;38(6):557- } \\
\text { 63. Epub 2003/11/25. }\end{array}$ & Excluded by abstract \\
\hline 164 & $\begin{array}{l}\text { Lamster IB, Rodrick ML, Sonis ST, Falchuk ZM. An analysis of peripheral blood and } \\
\text { salivary polymorphonuclear leukocyte function, circulating immune complex levels and } \\
\text { oral status in patients with inflammatory bowel disease. J Periodontol. 1982;53(4):231-8. } \\
\text { Epub 1982/04/01. }\end{array}$ & Excluded by abstract \\
\hline 165 & $\begin{array}{l}\text { Mendieta C, Reeve CM. Periodontal manifestations of systemic disease and management } \\
\text { of patients with systemic disease. Current opinion in periodontology. 1993:18-27. Epub } \\
\text { 1993/01/01. }\end{array}$ & Excluded by abstract \\
\hline 166 & $\begin{array}{l}\text { Sanchez AR, Rogers RS, 3rd, Sheridan PJ. Oral ulcerations are associated with the loss } \\
\text { of response to infliximab in Crohn's disease. Journal of oral pathology \& medicine. } \\
2005 ; 34(1): 53-5 \text {. }\end{array}$ & Excluded by abstract \\
\hline 167 & $\begin{array}{l}\text { Tabbaa M, Golubic M, Roizen MF, Bernstein AM. Docosahexaenoic acid, inflammation, } \\
\text { and bacterial dysbiosis in relation to periodontal disease, inflammatory bowel disease, } \\
\text { and the metabolic syndrome. Nutrients. 2013;5(8):3299-310. Epub 2013/08/24. }\end{array}$ & Excluded by abstract \\
\hline 168 & $\begin{array}{l}\text { Van Dyke TE, Dowell VR, Jr., Offenbacher S, Snyder W, Hersh T. Potential role of } \\
\text { microorganisms isolated from periodontal lesions in the pathogenesis of inflammatory } \\
\text { bowel disease. Infection and immunity. 1986;53(3):671-7. Epub 1986/09/01. }\end{array}$ & Excluded by abstract \\
\hline 169 & $\begin{array}{l}\text { Vrotsos JA, Vrahopoulos TP. Effects of systemic diseas } \\
\text { opinion in periodontology. 1996;3:19-26. Epub 1996/01// }\end{array}$ & Excluded by abstract \\
\hline 170 & $\begin{array}{l}\text { Andersen KM, Selvig KA, Leknes K. Altered healing following mucogingival surgery in a } \\
\text { patient with crohn's disease: A literature review and case report. Journal of } \\
\text { Periodontology. 2003;74(4):537-46. }\end{array}$ & Excluded by abstract \\
\hline 171 & $\begin{array}{l}\text { Brandtzaeg P. Inflammatory bowel disease: Clinics and pathology: Do inflammatory bowel } \\
\text { disease and periodontal disease have similar immunopathogeneses? Acta Odontologica } \\
\text { Scandinavica. } 2001 ; 59(4): 235-43 \text {. }\end{array}$ & Excluded by abstract \\
\hline 172 & $\begin{array}{l}\text { Engel LD, Pasquinelli KL, Leone SA, Moncla BJ, Nielson KD, Rabinovitch PS. Abnormal } \\
\text { lymphocyte profiles and leukotriene B4 status in a patient with Crohn's disease and } \\
\text { severe periodontitis. Journal of Periodontology. 1988;59(12):841-7. }\end{array}$ & Excluded by abstract \\
\hline 173 & $\begin{array}{l}\text { Indriolo A, Greco S, Ravelli P, Fagiuoli S. What can we learn about biofilm/host } \\
\text { interactions from the study of inflammatory bowel disease. Journal of Clinical } \\
\text { Periodontology. 2011;38(SUPPL. 11):36-43. }\end{array}$ & Excluded by abstract \\
\hline 174 & $\begin{array}{l}\text { Kalmar JR. Crohn's disease: orofacial considerations and disease pathogenesis. } \\
\text { Periodontology 2000. 1994;6:101-15. }\end{array}$ & Excluded by abstract \\
\hline 175 & $\begin{array}{l}\text { Kaner D, Bernimoulin JP, Dietrich T, Kleber BM, Friedmann A. Calprotectin levels in } \\
\text { gingival crevicular fluid predict disease activity in patients treated for generalized } \\
\text { aggressive periodontitis. Journal of Periodontal Research. } 2011 ; 46(4): 417-26 .\end{array}$ & Excluded by abstract \\
\hline 176 & $\begin{array}{l}\text { Leresche L, Dworkin SF. The role of stress in inflammatory disease, including periodontal } \\
\text { disease: Review of concepts and current findings. Periodontology 2000. 2002;30(1):91- } \\
\text { 103. }\end{array}$ & Excluded by abstract \\
\hline 177 & $\begin{array}{l}\text { Nibali L, Henderson B, Sadiq ST, Donos N. Genetic dysbiosis: The role of microbial } \\
\text { insults in chronic inflammatory diseases. Journal of Oral Microbiology. 2014;6(1). }\end{array}$ & Excluded by abstract \\
\hline 178 & $\begin{array}{l}\text { Scheper HJ, Brand HS. Oral aspects of Crohn's disease. International Dental Journal. } \\
2002 ; 52(3): 163-72 \text {. }\end{array}$ & $\mathrm{E}$ \\
\hline 179 & Mattos LFCd, Santos CdMMLd, Roxo MAP, Terezan MLF. Possível associação entre & Excluded by abstract \\
\hline
\end{tabular}




\begin{tabular}{|c|c|c|}
\hline & $\begin{array}{l}\text { doença periodontal e doenças intestinais inflamatórias crônicas. Possible association } \\
\text { between periodontal disease and chronic inflammatory bowel diseases. } \\
\text { Periodontia.24(3):17-23. }\end{array}$ & \\
\hline 180 & $\begin{array}{l}\text { Menegat JSB. Níveis elevados de IL-6 no fluido gengival de pacientes com periodontite } \\
\text { crônica e retrocolite ulcerativa idiopática. Elevated levels of IL-6 in the gingival crevicular } \\
\text { fluid from patients with chronic periodontitis and inflammatory bowel disease.37-. }\end{array}$ & Excluded by abstract \\
\hline 181 & $\begin{array}{l}\text { Miranda Rius J, Ramírez Rámiz A, Conde Mir I, Mendieta Fiter C. Manifestaciones orales } \\
\text { y periodontales de la enfermedad inflamatoria intestinal. Oral and periodontal } \\
\text { manifestations of inflammatory bowel disease. RCOE.6(3):287-92. }\end{array}$ & Excluded by abstract \\
\hline 182 & $\begin{array}{l}\text { Deas DE, Mackey SA, McDonnell HT. Systemic disease and periodontitis: manifestations } \\
\text { of neutrophil dysfunction. Periodontology } 2000.2003 ; 32: 82-104 \text {. }\end{array}$ & Excluded by abstract \\
\hline 183 & $\begin{array}{l}\text { Jones JV, Dowell VR, Place DA, Offenbacher S, Vandyke TE. Phenotypic Characteristics } \\
\text { of Motile, Nonspore-Forming, Gram-Negative Anaerobes from Periodontal Lesions of } \\
\text { Patients with Inflammatory Bowel-Disease. Journal of Dental Research. 1986;65:205-. }\end{array}$ & Excluded by abstract \\
\hline 184 & $\begin{array}{l}\text { Danilevskii NF, Zavernaia AM, Tkachuk NN, Mokhort VV. Characteristics of the course of } \\
\text { periodontal diseases in patients with nonspecific ulcerative colitis and Crohn's disease. } \\
\text { Stomatologiya 1987;66:20-2. }\end{array}$ & $\begin{array}{l}\text { Excluded; } \\
\text { irretrievable report. }\end{array}$ \\
\hline 185 & $\begin{array}{l}\text { Gatti S, Correa RRM, Macedo JMB, Menegat JSB, Fischer RG, Brito F, et al. [Presence } \\
\text { of TNFA gene polymorphism in patients with crohn's disease and chronic periodontitis] / } \\
\text { Presença do polimorfismo do gene TNFA em pacientes com doença de crohn e com } \\
\text { periodontite crônica. Periodontia 2012;22:63-9. }\end{array}$ & $\begin{array}{l}\text { Excluded; } \\
\text { irretrievable report. }\end{array}$ \\
\hline 186 & $\begin{array}{l}\text { Moraes SHd, Trento C, Moraes GF. [Oral manifestations of Crohn Disease] / } \\
\text { Manifestações bucais da Doença de Crohn. JBC J Bras Clin Estet Odontol 2001;5:45-7. }\end{array}$ & $\begin{array}{l}\text { d; } \\
\text { able report. }\end{array}$ \\
\hline 187 & $\begin{array}{l}\text { Pumphrey D, Dowell VR, Kuhn H, Jones J, Offenbacher S, Vandyke TE. The Lack of } \\
\text { Association of Oral Microbiota to Periodontal-Disease in Inflammatory Bowel-Disease } \\
\text { (IBD) Patients. J Dent Res 1987;66:226-226. }\end{array}$ & $\begin{array}{l}\text { Excluded; } \\
\text { irretrievable report. }\end{array}$ \\
\hline 188 & $\begin{array}{l}\text { Rikardsson S, Jonsson J, Hultin M, Gustafsson A, Johannsen A. Perceived oral health in } \\
\text { patients with Crohn's disease. Oral Health Prev Dent 2009;7:277-82. }\end{array}$ & $\begin{array}{l}\text { Excluded; } \\
\text { irretrievable }\end{array}$ \\
\hline 189 & $\begin{array}{l}\text { Toledo MF, Rode SDM, Lemos Júnior CA, Basile AP, Lotufo MA. Manifestações clínicas } \\
\text { intrabucais da doença de Crohn-Relato de caso clínico. Comun Ciênc Saúde } \\
\text { 2006;17:309-313. }\end{array}$ & $\begin{array}{l}\text { Excluded; } \\
\text { irretrievable report. }\end{array}$ \\
\hline 190 & $\begin{array}{l}\text { Louzeiro GC. Conhecimento dos estudantes de Odontologia sobre a doença celíaca e } \\
\text { suas manifestações bucais. } 2013 \text { Dissertation. }\end{array}$ & $\begin{array}{l}\text { irrelevant } \\
\text { ew subject. }\end{array}$ \\
\hline 191 & $\begin{array}{l}\text { Brito F, Pedreira RR, Fischer RG, Figueredto CMdS. [Interrelationship between } \\
\text { periodontal disease and Crohn's disease] / Inter-relação entre a doença periodontal e a } \\
\text { doença de Crohn. Rev Ciênc Méd Biol 2006;5:261-7. }\end{array}$ & Excluded; review. \\
\hline 192 & $\begin{array}{l}\text { Mattos LFC, Santos CMML, Roxo MAP, Terezan MLF. [Possible association between } \\
\text { periodontal disease and chronic inflammatory bowel diseases] / Possível associação } \\
\text { entre doença periodontal e doenças intestinais inflamatórias crônicas. Periodontia } \\
\text { 2014;24:17-23. }\end{array}$ & Exclu \\
\hline 193 & $\begin{array}{l}\text { Menegat J, Brito F, Barros F, Pedreira R, Fischer RG, Figueredo CMS. [High levels of IL- } \\
6 \text { in gingival crevicular fluid from patients with chronic periodontitis and ulcerative colitis] / } \\
\text { Níveis elevados de il-6 no fluido gengival de pacientes com periodontite crônica e } \\
\text { retrocolite ulcerativa idiopática. Periodontia 2009;20:61-8. }\end{array}$ & Excluded; review. \\
\hline 194 & $\begin{array}{l}\text { Lamster I, Sonis S, Hannigan A, Kolodkin A. An association between Crohn's disease, } \\
\text { periodontal disease and enhanced neutrophil function. J Periodontol 1978;49:475-9. }\end{array}$ & $\begin{array}{l}\text { Excluded; case } \\
\text { report. }\end{array}$ \\
\hline 195 & $\begin{array}{l}\text { Pincelli TPH. Manifestações orais da doença inflamatória intestinal: estudo clínico- } \\
\text { patológico retrospectivo. } 2010 \text { Disssertation. }\end{array}$ & $\begin{array}{l}\text { Excluded; case } \\
\text { report. }\end{array}$ \\
\hline 196 & $\begin{array}{l}\text { Flemmig TF, Shanahan F, Miyasaki KT. Prevalence and Severity of Periodontal-Disease } \\
\text { in Patients with Inflammatory Bowel-Disease. J Clin Periodontol 1991;18:690-7. }\end{array}$ & $\begin{array}{l}\text { Excluded; no IBD- } \\
\text { free patients. }\end{array}$ \\
\hline 197 & $\begin{array}{l}\text { Laranjeira N, Valido S, Meira T, Fonseca J, Freitas J. [Oral disorders in patients with } \\
\text { Inflammatory Bowel Disease. A pilot study] / Manifestações orais em doentes com } \\
\text { doença inflamatória intestinal. Estudo piloto. Colóquios Garcia de Orta 2015;2:1. }\end{array}$ & $\begin{array}{l}\text { Excluded; no IBD- } \\
\text { free patients. }\end{array}$ \\
\hline 198 & $\begin{array}{l}\text { Meurman JH, Halme L, Laine P, von Smitten K, Lindqvist C. Gingival and dental status, } \\
\text { salivary acidogenic bacteria, and yeast counts of patients with active or inactive Crohn's } \\
\text { disease. Oral Surg Oral Med Oral Pathol 1994;77:465-8. }\end{array}$ & $\begin{array}{l}\text { Excluded; no IBD- } \\
\text { free patients. }\end{array}$ \\
\hline $19 s$ & $\begin{array}{l}\text { Roriz JV. Manifestações bucais em pacientes com doenças gastrointestinais } \\
\text { inflamatórias. } 2008 \text { Dissertation. }\end{array}$ & $\begin{array}{l}\text { Excluded; no IBD- } \\
\text { free patients. }\end{array}$ \\
\hline 200 & Schulz S, Reichert S, Streetz K, Trautwein C, Reichert Y, Gläser C, et al. Tumor necrosis & Excluded; no IBD- \\
\hline
\end{tabular}




\begin{tabular}{|c|c|c|}
\hline & $\begin{array}{l}\text { factor-a and oral inflammation in patients with crohn disease. J Periodontol 2014;85:1424- } \\
31 .\end{array}$ & free patients. \\
\hline 201 & $\begin{array}{l}\text { Stein JM, Conrads G, Sooriyakumaran M, Lampert F, Smeets R, Weiss C, Lammert F: } \\
\text { Periodontal status of patients with Crohn's Disease. Int Poster J Dent Oral Med } \\
\text { 2009;11:439. }\end{array}$ & $\begin{array}{l}\text { Excluded; no IBD- } \\
\text { free patients. }\end{array}$ \\
\hline 202 & $\begin{array}{l}\text { Stein JM, Lammert F, Zimmer V, Granzow M, Reichert S, Schulz S, et al. Clinical } \\
\text { periodontal and microbiologic parameters in patients with crohn's disease with } \\
\text { consideration of the CARD15 genotype. J Periodontol 2010;81:535-45. }\end{array}$ & $\begin{array}{l}\text { Excluded; no IBD- } \\
\text { free patients. }\end{array}$ \\
\hline 203 & $\begin{array}{l}\text { Brito F, Zaltman C, Carvalho AT, Fischer RG, Persson R, Gustafsson A, et al. Subgingival } \\
\text { microflora in inflammatory bowel disease patients with untreated periodontitis. Eur J } \\
\text { Gastroenterol Hepatol 2013;25:239-45. }\end{array}$ & $\begin{array}{l}\text { Excluded; no } \\
\text { periodontal-free } \\
\text { patients. }\end{array}$ \\
\hline 204 & $\begin{array}{l}\text { Figueredo CM, Brito F, Barros FC, Menegat JSB, Pedreira RR, Fischer RG, et al. } \\
\text { Expression of cytokines in the gingival crevicular fluid and serum from patients with } \\
\text { inflammatory bowel disease and untreated chronic periodontitis. J Periodon Res } \\
2011 ; 46: 141-6 \text {. }\end{array}$ & $\begin{array}{l}\text { Excluded; no } \\
\text { periodontal-free } \\
\text { patients. }\end{array}$ \\
\hline 205 & $\begin{array}{l}\text { Rathnayake N. Pro-inflammatory mediators in gingival crevicular fluid of patients with } \\
\text { Inflammatory Bowel Disease, IBD. } 2008 \text { Dissertation }\end{array}$ & $\begin{array}{l}\text { Excluded; no } \\
\text { periodontal-free } \\
\text { patients. }\end{array}$ \\
\hline 206 & $\begin{array}{l}\text { Silva FdB. [Th cell profile in the gingival crevicular fluid from inflammatory bowel disease } \\
\text { patients with chronic periodontitis] / Perfil da resposta Th1/Th2 no fluido gengival de } \\
\text { pacientes portadores de doença inflamatória intestinal com periodontite crônica. 2008; } \\
\text { Doctoral Dissertation, Rio de Janeiro. }\end{array}$ & $\begin{array}{l}\text { Excluded; no } \\
\text { periodontal-free } \\
\text { patients. }\end{array}$ \\
\hline 207 & $\begin{array}{l}\text { Szymanska S, Lordal M, Rathnayake N, Gustafsson A, Johannsen A. Dental caries, } \\
\text { prevalence and risk factors in patients with Crohn's disease. PloS one 2014;9:e91059. }\end{array}$ & $\begin{array}{l}\text { Excluded; no } \\
\text { periodontal } \\
\text { outcomes. }\end{array}$ \\
\hline 208 & $\begin{array}{l}\text { Barros FCd, Brito F, Menegat JS, Pedreira RR, Carvalho ATP, Carneiro AJdV, et al. } \\
\text { [Prevalence of periodontitis in patients with inflammatory bowel disease] / Prevalência de } \\
\text { periodontite em pacientes com doença inflamatória intestinal. Periodontia 2008;18:44-8. }\end{array}$ & Included \\
\hline 209 & $\begin{array}{l}\text { Barros FCd. [Oral condition in patients with inflamatory bowel disease] / Condição oral em } \\
\text { pacientes com doença inflamatória intestinal. 2007; Master Thesis, Rio de Janeiro. }\end{array}$ & Included \\
\hline 210 & $\begin{array}{l}\text { Brito F, Barros FCD, Zaltman C, Pugas Carvalho AT, De Vasconcellos Carneiro AJ, } \\
\text { Fischer RG, et al. Prevalence of periodontitis and DMFT index in patients with Crohn's } \\
\text { disease and ulcerative colitis. J Clin Periodontol 2008;35:555-60. } \\
\end{array}$ & Included \\
\hline 211 & $\begin{array}{l}\text { Brito F, Zaltman C, Carvalho AT, Fischer RG, Persson R, Gustafsson A, et al. Subgingival } \\
\text { microflora in inflammatory bowel disease patients with untreated periodontitis. Eur J } \\
\text { Gastroenterol Hepatol 2013;25:239-45. }\end{array}$ & Included manually. \\
\hline 212 & $\begin{array}{l}\text { Figueredo CM, Brito F, Barros FC, Menegat JSB, Pedreira RR, Fischer RG, et al. } \\
\text { Expression of cytokines in the gingival crevicular fluid and serum from patients with } \\
\text { inflammatory bowel disease and untreated chronic periodontitis. J Periodon Res } \\
2011 ; 46: 141-6 \text {. }\end{array}$ & Included manually. \\
\hline 213 & $\begin{array}{l}\text { Grössner-Schreiber B, Fetter T, Hedderich J, Kocher T, Schreiber S, Jepsen S. } \\
\text { Prevalence of dental caries and periodontal disease in patients with inflammatory bowel } \\
\text { disease: A case-control study. J Clin Periodontol 2006;33:478-84. }\end{array}$ & Included \\
\hline 214 & $\begin{array}{l}\text { Habashneh RA, Khader YS, Alhumouz MK, Jadallah K, Ajlouni Y. The association } \\
\text { between inflammatory bowel disease and periodontitis among Jordanians: A case-control } \\
\text { study. J Periodont Res 2012;47:293-8. }\end{array}$ & Included \\
\hline 215 & $\begin{array}{l}\text { Silva FdB. [Th cell profile in the gingival crevicular fluid from inflammatory bowel disease } \\
\text { patients with chronic periodontitis] / Perfil da resposta Th1/Th2 no fluido gengival de } \\
\text { pacientes portadores de doença inflamatória intestinal com periodontite crônica. 2008; } \\
\text { Doctoral Dissertation, Rio de Janeiro. }\end{array}$ & Included manually. \\
\hline 216 & $\begin{array}{l}\text { Rathnayake N. Pro-inflammatory mediators in gingival crevicular fluid of patients with } \\
\text { Inflammatory Bowel Disease, IBD. } 2008 \text { Dissertation. }\end{array}$ & Included manually. \\
\hline 217 & $\begin{array}{l}\text { Ślebioda Z, Szponar E, Linke K. Comparative analysis of the oral cavity status in patients } \\
\text { with Crohn's disease and ulcerative colitis. J Stomatology } 2011 ; 64: 212-24 \text {. }\end{array}$ & Included \\
\hline 218 & $\begin{array}{l}\text { Van Dyke TE, Dowell VR, Jr., Offenbacher S, Snyder W, Hersh T. Potential role of } \\
\text { microorganisms isolated from periodontal lesions in the pathogenesis of inflammatory } \\
\text { bowel disease. Infection and immunity. 1986;53(3):671-7. Epub 1986/09/01. }\end{array}$ & Included \\
\hline 219 & $\begin{array}{l}\text { Vavricka SR, Manser CN, Hediger S, Vogelin M, Scharl M, Biedermann L, et al. } \\
\text { Periodontitis and gingivitis in inflammatory bowel disease: a case-control study. Inflamm }\end{array}$ & Included \\
\hline
\end{tabular}


Bowel Dis 2013;19:2768-77.

Agossa K, Dendooven A, Dubuquoy L, Gower-Rousseau C, Delcourt-Debruyne E,

1 Capron M. Periodontal manifestations of inflammatory bowel disease: Emerging epidemiologic and biologic evidence. Journal of Periodontal Research. 2016.

Included

Badran Z, Struillou X, Verner C, Clee T, Rakic M, Martinez MC, et al. Periodontitis as a

2 risk factor for systemic disease: Are microparticles the missing link? Medical hypotheses. 2015;84(6):555-6. Epub 2015/03/10.

Do MP, Neut C, Metz H, Delcourt E, Siepmann J, Mader K, et al. Mechanistic analysis of

$3 \quad$ PLGA/HPMC-based in-situ forming implants for periodontitis treatment. European journal of pharmaceutics and biopharmaceutics : official journal of Arbeitsgemeinschaft fur Pharmazeutische Verfahrenstechnik eV. 2015;94:273-83. Epub 2015/06/07.

Keskin M, Zeidan-Chulia F, Gursoy M, Kononen E, Rautava J, Gursoy UK. Two Cheers

4 for Crohn's Disease and Periodontitis: Beta-Defensin-2 as an Actionable Target to Intervene on Two Clinically Distinct Diseases. Omics : a journal of integrative biology. 2015;19(8):443-50. Epub 2015/07/15.

Zwicker S, Martinez GL, Bosma M, Gerling M, Clark R, Majster M, et al. Interleukin 34: a

5 new modulator of human and experimental inflammatory bowel disease. Clinical science (London, England : 1979). 2015;129(3):281-90. Epub 2015/04/22.

Al-Dabbagh N. Comparison of Post Tooth Extraction Healing Using different Xenograft

$6 \quad$ Materials -A Pilot Study [M.S.]. Ann Arbor: Tufts University School of Dental Medicine; 2015.

Anderson AP. The association between physical activity and serum immunoglobulin $\mathrm{G}$

7 (IgG) antibodies against periodontal bacteria [M.S.P.H.]. Ann Arbor: University of South Carolina; 2016.

8 Anderson WD, 3rd, Treister NS, Mayeaux EJ, Jr., Nalliah RP. Oral lesions you can't afford to miss. The Journal of family practice. 2015;64(7):392-9. Epub 2015/09/02.

Excluded by abstract

Excluded by abstract

Excl

Excluded by abstract

Elburki MS. A Novel Chemically Modified Curcumin as a Pleiotropic MMP-Inhibitor:

$9 \quad$ Therapeutic Potential in Locally- and Systemically-Induced Periodontal (and other)

$9 \quad$ Connective Tissue Breakdown [Ph.D.]. Ann Arbor: State University of New York at Stony Brook; 2015.

10 Fine DH. Lactoferrin: A Roadmap to the Borderland between Caries and Periodontal

\begin{tabular}{ll}
10 & Disease. Journal of dental research. 2015;94(6):768-76. Epub 2015/03/19. \\
\hline 11 & Goldberg S. The role of Rac and Filamin A in osteoclast function and osteoclast
\end{tabular}

Excluded by abstract

Excluded by abstract

Excluded by title

Excluded by title

Excluded by title

11 heterogeneity [Ph.D.]. Ann Arbor: University of Toronto (Canada); 2015.

12 Kalthoum ZE. The effect of vitamin D on coronal and root caries [D.Sc.D.]. Ann Arbor: Boston University; 2016.

Kumar A, Thotakura PL, Tiwary BK, Krishna R. Target identification in Fusobacterium

13 nucleatum by subtractive genomics approach and enrichment analysis of host-pathogen protein-protein interactions. BMC microbiology. 2016;16:84. Epub 2016/05/14.

Lee S, Lee J, Ha J, Choi Y, Kim S, Lee H, et al. Clinical relevance of infections with

14 zoonotic and human oral species of Campylobacter. Journal of microbiology (Seoul, Korea). 2016;54(7):459-67. Epub 2016/06/29.

Lundmark A, Davanian H, Bage T, Johannsen G, Koro C, Lundeberg J, et al.

15 Transcriptome analysis reveals mucin 4 to be highly associated with periodontitis and identifies pleckstrin as a link to systemic diseases. Scientific reports. 2015;5:18475.

16 Mai X. Periodontal disease, the oral microbiome, and their associations with cancer risk 2015.

Martinez RC, Bedani R, Saad SM. Scientific evidence for health effects attributed to the

17 consumption of probiotics and prebiotics: an update for current perspectives and future challenges. The British journal of nutrition. 2015;114(12):1993-2015. Epub 2015/10/08.

18 Puri S. Are Bone Turnover Markers and Vitamin D levels associated with Frequency of Complete Denture Relines? [M.S.]. Ann Arbor: Loma Linda University; 2015.

Excluded by title

Excluded by title

Excluded by title

Excluded by title

Excluded by title

Excluded by title

Excluded by title

Excluded by title

Excluded by title

Reinhard A, Sandborn WJ, Melhem H, Bolotine L, Chamaillard M, Peyrin-Biroulet L.

Photodynamic therapy as a new treatment modality for inflammatory and infectious

Excluded by title

Excluded by title 


\begin{tabular}{|c|c|c|}
\hline & conditions. Expert review of clinical immunology. 2015;11(5):637-57. Epub 2015/04/04. & \\
\hline 20 & $\begin{array}{l}\text { Schnitzler F, Friedrich M, Stallhofer J, Schonermarck U, Fischereder M, Habicht A, et al. } \\
\text { Solid Organ Transplantation in Patients with Inflammatory Bowel Diseases (IBD): Analysis } \\
\text { of Transplantation Outcome and IBD Activity in a Large Single Center Cohort. PloS one. } \\
2015 ; 10(8): e 0135807 \text {. Epub 2015/08/20. }\end{array}$ & Excluded by title \\
\hline 21 & $\begin{array}{l}\text { Schnitzler F, Friedrich M, Wolf C, Stallhofer J, Angelberger M, Diegelmann J, et al. The } \\
\text { NOD2 Single Nucleotide Polymorphism rs } 72796353 \text { (IVS4+10 A>C) Is a Predictor for } \\
\text { Perianal Fistulas in Patients with Crohn's Disease in the Absence of Other NOD2 } \\
\text { Mutations. PloS one. } 2015 ; 10(7): e 0116044 \text {. Epub } 2015 / 07 / 07 .\end{array}$ & Excluded by title \\
\hline 22 & $\begin{array}{l}\text { Slaton K. 16S rRNA gene sequencing to evaluate the effects of six commonly prescribed } \\
\text { antibiotics [M.S.]. Ann Arbor: The University of Alabama at Birmingham; } 2016 \text {. }\end{array}$ & Excluded by title \\
\hline 23 & $\begin{array}{l}\text { Thomas LV, Suzuki K, Zhao J. Probiotics: a proactive approach to health. A symposium } \\
\text { report. The British journal of nutrition. } 2015 ; 114 \text { Suppl 1:S1-15. Epub } 2015 / 11 / 10 \text {. }\end{array}$ & Excluded by title \\
\hline 24 & $\begin{array}{l}\text { Tsuchiya S, Sugimoto K, Omori M, Matsuda R, Fujio M, Hibi H. Mandibular osteomyelitis } \\
\text { implicated in infliximab and periapical periodontitis: A case report. Journal of Oral and } \\
\text { Maxillofacial Surgery, Medicine, and Pathology. } 2016 ; 28(5): 410-5 \text {. }\end{array}$ & Excluded by title \\
\hline 25 & $\begin{array}{l}\text { Vinesh E, Masthan KMK, Sathish Kumar M, Marytresa Jeyapriya S, Babu A, Thinakaran } \\
\text { M. A clinicopathologic study of oral changes in gastroesophageal reflux disease, gastritis, } \\
\text { and ulcerative colitis. Journal of Contemporary Dental Practice. } 2016 ; 17(11): 943-7 \text {. }\end{array}$ & Excluded by title \\
\hline 26 & $\begin{array}{l}\text { Kłaniecka B, Kaczmarek U. Oral cavity condition and selected salivary parameters in } \\
\text { children and adolescents suffering from inflammatory bowel diseases. Dental and Medical } \\
\text { Problems. } 2016 ; 53(2): 210-5 \text {. }\end{array}$ & $\begin{array}{l}\text { Excluded; } \\
\text { irretrievable report. }\end{array}$ \\
\hline 27 & $\begin{array}{l}\text { Lovelina FD. Oral health status of ulcerative colitis patients: A comparative study. } \\
\text { Inflammatory Bowel Diseases Conference: } 2015 \text { Advances in Inflammatory Bowel } \\
\text { Diseases, Crohn's and Colitis Foundation's National Clinical and Research Conference, } \\
\text { AIBD } 2015 \text { Orlando, FL United States Conference Start: } 20151210 \text { Conference End: } \\
20151212 \text { Conference Publication: (varpagings) [Internet]. 2016:[S24 p.]. Available from: } \\
\text { http://onlinelibrary.wiley.com/o/cochrane/clcentral/articles/819/CN-01171819/frame.html. }\end{array}$ & $\begin{array}{l}\text { Excluded; } \\
\text { irretrievable report. }\end{array}$ \\
\hline 28 & $\begin{array}{l}\text { S.afFernandes-Costa AN, Universidade Federal do Rio Grande do NorteMaia JMdC, } \\
\text { Universidade Federal do Rio Grande do NorteMedeiros AMCd. Prevalência de alterações } \\
\text { bucais nas doenças gastrointestinais inflamatórias crônicas: análise de } 10 \text { casos. Rev } \\
\text { ciênc plur. 2016;1(1):57-64. }\end{array}$ & $\begin{array}{l}\text { Excluded; case } \\
\text { series. }\end{array}$ \\
\hline 29 & $\begin{array}{l}\text { Menegat JSB, Lira-Junior R, Siqueira MA, Brito F, Carvalho AT, Fischer RG, et al. } \\
\text { Cytokine expression in gingival and intestinal tissues of patients with periodontitis and } \\
\text { inflammatory bowel disease: An exploratory study. Arch Oral Biol 2016;66:141-6. }\end{array}$ & $\begin{array}{l}\text { Excluded; no } \\
\text { periodontal } \\
\text { outcomes. }\end{array}$ \\
\hline 30 & $\begin{array}{l}\text { Johannsen A, Fored MC, Hakansson J, Ekbom A, Gustafsson A. Consumption of dental } \\
\text { treatment in patients with inflammatory bowel disease, a register study. PloS one. } \\
2015 ; 10(8): \mathrm{e} 0134001 .\end{array}$ & $\begin{array}{l}\text { Excluded; no } \\
\text { periodontal } \\
\text { outcomes. }\end{array}$ \\
\hline 31 & $\begin{array}{l}\text { Koutsochristou V, Zellos A, Dimakou K, Panayotou I, Siahanidou S, Roma-Giannikou E, } \\
\text { et al. Dental Caries and Periodontal Disease in Children and Adolescents with } \\
\text { Inflammatory Bowel Disease: A Case-Control Study. Inflamm Bowel Dis 2015;21(8):1839- } \\
46 .\end{array}$ & Included \\
\hline
\end{tabular}


Appendix S4 Results of the individual outcomes from the included studies that were not included in meta-analyses.

\begin{tabular}{|c|c|c|c|c|c|c|c|}
\hline \multirow[b]{2}{*}{ Outcome } & \multirow[b]{2}{*}{ Study } & \multicolumn{2}{|c|}{ IBD vs control } & \multicolumn{2}{|c|}{ CD vs control } & \multicolumn{2}{|c|}{ UC vs control } \\
\hline & & MD (95\% Cl) & $\mathbf{P}$ & MD (95\% Cl) & $\mathbf{P}$ & MD $(95 \% \mathrm{Cl})$ & $\mathbf{P}$ \\
\hline SG shallow pockets: IL-4 & Barros 2007 & $-0.85(-1.71,0.01)$ & 0.052 & $-0.50(-1.47,0.47)$ & 0.312 & $-1.20(-2.04,-0.36)$ & 0.005 \\
\hline Serum IFN-y & Barros 2007 & $0.00(-0.16,0.16)$ & 1.000 & $0.00(-0.17,0.17)$ & 1.000 & $0.00(-0.15,0.15)$ & 1.000 \\
\hline Serum IL-10 & Barros 2007 & $-1.80(-11.37,7.77)$ & 0.712 & $-3.40(-11.32,4.52)$ & 0.400 & $-0.30(-13.55,12.95)$ & 0.965 \\
\hline Serum IL-12p70 & Barros 2007 & $0.00(-1.24,1.24)$ & 1.000 & $0.00(-1.24,1.24)$ & 1.000 & $0.00(-1.24,1.24)$ & 1.000 \\
\hline Serum IL-18 & Barros 2007 & $50.71(10.26,91.16)$ & 0.014 & $48.90(-4.31,102.11)$ & 0.072 & $52.40(3.77,101.03)$ & 0.035 \\
\hline Serum IL-4 & Barros 2007 & $0.00(-4.56,4.56)$ & 1.000 & $0.00(-4.44,4.44)$ & 1.000 & $0.00(-5.65,5.65)$ & 1.000 \\
\hline Serum IL-6 & Barros 2007 & $0.71(-7.12,8.54)$ & 0.859 & $0.40(-7.60,8.40)$ & 0.922 & $1.00(-6.90,8.90)$ & 0.804 \\
\hline SG deep pockets: \%PI & Barros 2007 & $-5.45(-29.21,18.31)$ & 0.653 & $-0.50(-27.25,26.25)$ & 0.971 & $-10.40(-37.79,16.99)$ & 0.457 \\
\hline SG deep pockets: CAL & Barros 2007 & $0.40(-0.69,1.49)$ & 0.470 & $0.60(-0.85,2.05)$ & 0.417 & $0.20(-0.88,1.28)$ & 0.717 \\
\hline SG deep pockets: GCF volume & Barros 2007 & $0.35(-0.36,1.06)$ & 0.337 & $0.30(-0.45,1.05)$ & 0.434 & $0.40(-0.54,1.34)$ & 0.405 \\
\hline SG deep pockets: IFN-Y & Barros 2007 & $0.00(-0.05,0.05)$ & 1.000 & $0.00(-0.08,0.08)$ & 1.000 & $0.00(-0.08,0.08)$ & 1.000 \\
\hline SG deep pockets: IL-18 & Barros 2007 & $4.85(-8.59,18.29)$ & 0.479 & $2.70(-11.88,17.28)$ & 0.717 & $7.00(-7.72,21.72)$ & 0.351 \\
\hline SG deep pockets: IL-1b & Barros 2007 & $1.00(-0.35,2.35)$ & 0.147 & $1.20(-0.21,2.61)$ & 0.095 & $0.80(-1.39,2.99)$ & 0.473 \\
\hline SG deep pockets: IL-4 & Barros 2007 & $-0.95(-1.99,0.09)$ & 0.074 & $-1.00(-2.05,0.05)$ & 0.061 & $-0.90(-2.07,0.27)$ & 0.130 \\
\hline SG deep pockets: IL-6 & Barros 2007 & $0.15(-0.09,0.39)$ & 0.212 & $0.00(-0.36,0.36)$ & 1.000 & $0.30(0.05,0.55)$ & 0.018 \\
\hline SG deep pockets: PPD & Barros 2007 & $-0.05(-0.92,0.82)$ & 0.911 & $-0.10(-1.14,0.94)$ & 0.850 & $0.00(-0.91,0.91)$ & 1.000 \\
\hline SG gingivitis: P.a. & Barros 2007 & $0.16(-0.04,0.36)$ & 0.121 & $0.37(0.08,0.66)$ & 0.013 & $-0.05(-0.24,0.14)$ & 0.597 \\
\hline SG gingivitis: Pa.m. & Barros 2007 & $0.05(-0.51,0.61)$ & 0.860 & $0.49(-0.23,1.21)$ & 0.181 & $-0.40(-0.93,0.13)$ & 0.137 \\
\hline SG gingivitis: Pr.m. & Barros 2007 & $0.49(0.10 .0 .88)$ & 0.014 & $0.97(0.45,1.49)$ & $<0.001$ & $0.01(-0.34,0.36)$ & 0.955 \\
\hline SG gingivitis: Sta.a & Barros 2007 & $0.75(0.27,1.23)$ & 0.002 & $1.40(0.79,2.01)$ & $<0.001$ & $0.10(-0.43,0.63)$ & 0.710 \\
\hline SG gingivitis: Str.a. & Barros 2007 & $0.29(-0.01,0.59)$ & 0.060 & $0.68(0.18,1.18)$ & 0.007 & $-0.10(-0.25,0.05)$ & 0.192 \\
\hline SG gingivitis: Str.mi. & Barros 2007 & $0.08(-0.20,0.36)$ & 0.574 & $0.34(-0.08,0.76)$ & 0.113 & $-0.19(-0.38,0.00)$ & 0.051 \\
\hline SG gingivitis: Str.mu. & Barros 2007 & $0.19(-0.20,0.58)$ & 0.342 & $0.49(-0.17,1.15)$ & 0.144 & $-0.11(-0.36,0.14)$ & 0.378 \\
\hline SG gingivitis: Tr.d. & Barros 2007 & $0.21(-0.08,0.50)$ & 0.151 & $0.39(-0.09,0.87)$ & 0.108 & $0.02(-0.17,0.21)$ & 0.833 \\
\hline SG non-smokers: BOP & Barros 2007 & $-2.80(-9.05,3.45)$ & 0.380 & $-3.40(-10.00,3.20)$ & 0.313 & $-1.80(-8.98,5.38)$ & 0.623 \\
\hline
\end{tabular}




\begin{tabular}{|c|c|c|c|c|c|c|c|}
\hline SG non-smokers: CAL & Barros 2007 & $0.11(-0.12,0.34)$ & 0.356 & $0.00(-0.23,0.23)$ & 1.000 & $0.30(-0.07,0.67)$ & 0.116 \\
\hline SG non-smokers: CAL>3 & Barros 2007 & $3.07(-3.36,9.50)$ & 0.349 & $1.30(-4.65,7.25)$ & 0.668 & $6.00(-4.79,16.79)$ & 0.276 \\
\hline SG non-smokers: DMFT & Barros 2007 & $1.77(-0.53,4.07)$ & 0.131 & $0.90(-1.60,3.40)$ & 0.480 & $3.20(0.29,6.11)$ & 0.031 \\
\hline SG non-smokers: no of teeth & Barros 2007 & $-1.38(-3.16,0.40)$ & 0.128 & $-1.00(-3.01,1.01)$ & 0.330 & $-2.00(-4.62,0.62)$ & 0.135 \\
\hline SG non-smokers: PI & Barros 2007 & $-13.90(-24.78,-3.02)$ & 0.012 & $-14.20(-24.97,-3.44)$ & 0.010 & $-13.40(-33.12,6.32)$ & 0.183 \\
\hline SG non-smokers: PPD & Barros 2007 & $0.86(0.72,1.00)$ & $<0.001$ & $0.90(0.69,1.11)$ & $<0.001$ & $0.80(0.71,0.89)$ & $<0.001$ \\
\hline SG periodontitis: B.u. & Barros 2007 & $0.17(0.07,0.27)$ & 0.001 & $0.30(0.16,0.44)$ & $<0.001$ & $0.03(-0.05,0.11)$ & 0.457 \\
\hline SG periodontitis: C.g. & Barros 2007 & $0.22(-0.06,0.50)$ & 0.127 & $0.44(0.09,0.79)$ & 0.014 & $-0.01(-0.30,0.28)$ & 0.946 \\
\hline SG periodontitis: P.m. & Barros 2007 & $0.42(-0.00,0.84)$ & 0.052 & $0.86(0.22,1.50)$ & 0.009 & $-0.02(-0.32,0.28)$ & 0.896 \\
\hline SG periodontitis: S.ang. & Barros 2007 & $0.35(0.06,0.64)$ & 0.018 & $0.77(0.36,1.18)$ & $<0.001$ & $-0.07(-0.26,0.12)$ & 0.459 \\
\hline SG periodontitis: S.aur. & Barros 2007 & $0.40(-0.06,0.86)$ & 0.091 & $0.83(0.16,1.50)$ & 0.015 & $-0.04(-0.39,0.31)$ & 0.823 \\
\hline SG periodontitis: S.int. & Barros 2007 & $0.21(0.01,0.42)$ & 0.044 & $0.43(0.14,0.72)$ & 0.004 & $-0.01(-0.18,0.16)$ & 0.906 \\
\hline SG periodontitis: S.mit. & Barros 2007 & $0.16(-0.06,0.38)$ & 0.145 & $0.34(0.07,0.61)$ & 0.012 & $-0.03(-0.23,0.17)$ & 0.766 \\
\hline SG periodontitis: S.mut. & Barros 2007 & $0.22(-0.13,0.57)$ & 0.218 & $0.54(0.04,1.04)$ & 0.033 & $-0.10(-0.37,0.17)$ & 0.468 \\
\hline SG shallow pockets: CAL & Barros 2007 & $-0.25(-0.37,-0.13)$ & $<0.001$ & $-0.30(-0.46,-0.14)$ & $<0.001$ & $-0.20(-0.36,-0.04)$ & 0.014 \\
\hline SG shallow pockets: GCF volume & Barros 2007 & $0.05(-0.50,0.60)$ & 0.859 & $0.10(-0.51,0.71)$ & 0.748 & $0.00(-0.73,0.73)$ & 1.000 \\
\hline SG shallow pockets: IFN-Y & Barros 2007 & $0.00(-0.05,0.05)$ & 1.000 & $0.00(-0.08,0.08)$ & 1.000 & $0.00(-0.08,0.08)$ & 1.000 \\
\hline SG shallow pockets: IL-18 & Barros 2007 & $-7.75(-24.54,9.04)$ & 0.365 & $-10.70(-27.33,5.93)$ & 0.207 & $-4.80(-23.08,13.48)$ & 0.607 \\
\hline SG shallow pockets: IL-1b & Barros 2007 & $-0.65(-1.47,0.17)$ & 0.122 & $-0.80(-1.69,0.09)$ & 0.079 & $-0.50(-1.39,0.39)$ & 0.272 \\
\hline SG shallow pockets: IL-6 & Barros 2007 & $0.00(-0.13,0.13)$ & 1.000 & $0.00(-0.00,0.00)$ & 1.000 & $0.00(-0.26,0.26)$ & 1.000 \\
\hline SG shallow pockets: PI & Barros 2007 & $-21.60(-44.76,1.56)$ & 0.068 & $-26.00(-54.59,2.59)$ & 0.075 & $-17.20(-43.47,9.07)$ & 0.199 \\
\hline SG shallow pockets: PPD & Barros 2007 & $-0.25(-0.41,-0.09)$ & 0.002 & $-0.20(-0.38,-0.02)$ & 0.032 & $-0.30(-0.53,-0.07)$ & 0.009 \\
\hline SG smokers: BOP & Barros 2007 & $-5.87(-15.93,4.19)$ & 0.253 & $-7.70(-18.38,2.98)$ & 0.157 & $-4.30(-15.22,6.62)$ & 0.440 \\
\hline SG smokers: CAL & Barros 2007 & $-0.03(-0.54,0.48)$ & 0.907 & $-0.30(-0.83,0.23)$ & 0.265 & $0.20(-0.35,0.75)$ & 0.479 \\
\hline SG smokers: CAL>3 & Barros 2007 & $5.19(-9.72,20.10)$ & 0.495 & $0.40(-15.09,15.89)$ & 0.960 & $9.30(-6.60,25.20)$ & 0.252 \\
\hline SG smokers: DMFT & Barros 2007 & $4.10(1.38,6.82)$ & 0.003 & $4.80(1.67,7.93)$ & 0.003 & $3.50(0.51,6.49)$ & 0.022 \\
\hline SG smokers: no of teeth & Barros 2007 & $-1.88(-4.26,0.50)$ & 0.121 & $0.00(-3.19,3.19)$ & 1.000 & $-3.50(-6.01,-0.99)$ & 0.006 \\
\hline SG smokers: PI & Barros 2007 & $-12.96(-29.87,3.95)$ & 0.133 & $-13.50(-32.09,5.09)$ & 0.155 & $-12.50(-31.25,6.25)$ & 0.191 \\
\hline SG smokers: PPD & Barros 2007 & $0.41(0.14,0.68)$ & 0.003 & $0.30(-0.17,0.77)$ & 0.210 & $0.50(0.29,0.71)$ & $<0.001$ \\
\hline
\end{tabular}




\begin{tabular}{|c|c|c|c|c|c|c|c|}
\hline TNFa & Barros 2007 & $-0.82(-2.73,1.09)$ & 0.400 & $-0.30(-2.43,1.83)$ & 0.783 & $-1.30(-3.26,0.66)$ & 0.194 \\
\hline DMFS & Groessner 2006 & $7.60(-2.77,17.97)$ & 0.151 & - & - & - & - \\
\hline Furcation involvement & Groessner 2006 & $7.90(-3.82,19.62)$ & 0.186 & - & - & - & - \\
\hline $\mathrm{CAL}>4$ & Habasneh 2012 & $15.66(10.41,20.91)$ & $<0.001$ & $12.25(4.76,19.74)$ & 0.001 & $17.65(11.23,24.07)$ & $<0.001$ \\
\hline CAL $>5$ & Habasneh 2012 & $3.51(1.58,5.44)$ & $<0.001$ & $0.03(-2.12,2.18)$ & 0.978 & $5.55(2.86,8.24)$ & $<0.001$ \\
\hline $\mathrm{Gl}$ & Habasneh 2012 & $0.94(0.77,1.11)$ & $<0.001$ & $0.88(0.66,1.10)$ & $<0.001$ & $0.97(0.79,1.15)$ & $<0.001$ \\
\hline GR & Habasneh 2012 & $0.30(0.14,0.46)$ & $<0.001$ & $0.09(-0.09,0.27)$ & 0.335 & $0.42(0.24,0.60)$ & $<0.001$ \\
\hline SG gingivitis: CAL & Rathnayake 2008 & $-0.15(-0.31,0.01)$ & 0.064 & $-0.30(-0.50,-0.10)$ & 0.004 & $0.00(-0.20,0.20)$ & 1.000 \\
\hline SG gingivitis: GCF & Rathnayake 2008 & $0.05(-0.36,0.46)$ & 0.809 & $0.10(-0.35,0.55)$ & 0.664 & $0.00(-0.54,0.54)$ & 1.000 \\
\hline SG gingivitis: PPD & Rathnayake 2008 & $-0.40(-0.64,-17)$ & 0.001 & $-0.30(-0.54,-0.06)$ & 0.015 & $-0.50(-0.84,-0.17)$ & 0.003 \\
\hline SG gingivitis: VPI & Rathnayake 2008 & $-45.00(-75.18,-14.82)$ & 0.003 & $-50.00(-91.91,-8.09)$ & 0.019 & $-40.00(-75.37,-4.64)$ & 0.027 \\
\hline SG periodontitis: CAL & Rathnayake 2008 & $0.85(0.14,1.56)$ & 0.019 & $0.70(-0.41,1.81)$ & 0.216 & $1.00(0.36,1.64)$ & 0.002 \\
\hline SG periodontitis: GCF & Rathnayake 2008 & $0.35(-0.18,0.88)$ & 0.194 & $0.30(-0.26,0.86)$ & 0.290 & $0.40(-0.30,1.10)$ & 0.260 \\
\hline SG periodontitis: PPD & Rathnayake 2008 & $0.20(-0.27,0.67)$ & 0.406 & $0.20(-0.44,0.84)$ & 0.538 & $0.20(-0.37,0.77)$ & 0.492 \\
\hline SG periodontitis: VPI & Rathnayake 2008 & $-11.65(-46.29,22.99)$ & 0.510 & $-8.30(-48.06,31.46)$ & 0.682 & $-15.00(-56.11,26.11)$ & 0.475 \\
\hline $\mathrm{D}$ (ecayed) index & Slebioda 2011 & $1.57(0.91,2.23)$ & $<0.001$ & $1.70(0.90,2.50)$ & $<0.001$ & $1.20(0.31,2.09)$ & 0.008 \\
\hline Dental caries treatment index & Slebioda 2011 & $-0.15(-0.24,-0.06)$ & 0.002 & $-0.18(-0.28,-0.08)$ & 0.000 & $-0.08(-0.23,0.07)$ & 0.308 \\
\hline Fillings & Slebioda 2011 & $-0.20(-1.57,1.17)$ & 0.775 & $-0.20(-1.67,1.27)$ & 0.790 & $-0.20(-2.24,1.84)$ & 0.848 \\
\hline$M$ (issing) index & Slebioda 2011 & $5.37(3.41,7.33)$ & $<0.001$ & $5.40(3.16,7.65)$ & $<0.001$ & $5.30(1.81,8.79)$ & 0.003 \\
\hline Oral hygiene index & Slebioda 2011 & $1.24(0.99,1.49)$ & $<0.001$ & $1.40(1.14,1.66)$ & $<0.001$ & $0.80(0.41,1.19)$ & $<0.001$ \\
\hline Bleeding in deepest tooth & Vavricka 2013 & $0.12(0.03,0.21)$ & 0.012 & $0.16(0.04,0.28)$ & 0.008 & $0.05(-0.07,0.17)$ & 0.406 \\
\hline CAL in deepest tooth & Vavricka 2013 & $0.43(0.11,0.75)$ & 0.009 & $0.43(0.04,0.83)$ & 0.033 & $0.44(-0.01,0.89)$ & 0.055 \\
\hline Papilla bleeding index & Vavricka 2013 & $0.23(0.12,0.34)$ & $<0.001$ & $0.23(0.11,0.35)$ & $<0.001$ & $0.22(0.03,0.41)$ & 0.023 \\
\hline Outcome & Study & OR $(95 \% \mathrm{Cl})$ & $\mathbf{P}$ & OR (95\% Cl) & $\mathbf{P}$ & OR (95\% Cl) & $\mathbf{P}$ \\
\hline CAL $>4$ & Groessner 2006 & $2.30(1.01,5.26)$ & 0.048 & - & - & - & - \\
\hline$C A L>5$ & Groessner 2006 & $2.01(0.97,4.16)$ & 0.060 & - & - & - & - \\
\hline Dentine caries & Groessner 2006 & $2.39(1.08,5.31)$ & 0.032 & - & - & - & - \\
\hline Atrophic tongue & Slebioda 2011 & $13.70(0.78,241.42)$ & 0.074 & $19.18(1.09,339.03)$ & 0.044 & - & - \\
\hline
\end{tabular}




\begin{tabular}{|c|c|c|c|c|c|c|c|}
\hline Candida & Slebioda 2011 & $3.09(1.62,5.87)$ & 0.001 & $3.24(1.62,6.47)$ & 0.001 & $2.70(1.06,6.90)$ & 0.038 \\
\hline Candida + & Slebioda 2011 & $0.98(0.46,2.08)$ & 0.953 & $0.84(0.37,1.92)$ & 0.673 & $1.43(0.50,4.05)$ & 0.505 \\
\hline Candida ++ & Slebioda 2011 & $1.30(0.48,3.50)$ & 0.601 & $1.68(0.61,4.62)$ & 0.316 & $0.38(0.04,3.21)$ & 0.371 \\
\hline Candida +++ & Slebioda 2011 & $7.13(2.05,24.86)$ & 0.002 & $6.62(1.83,23.90)$ & 0.004 & $8.69(2.04,36.99)$ & 0.003 \\
\hline Candida wz & Slebioda 2011 & $8.57(0.47,157.58)$ & 0.148 & $11.84(0.64,218.33)$ & 0.097 & - & - \\
\hline Complete dentition & Slebioda 2011 & $0.18(0.09,0.35)$ & $<0.001$ & $0.19(0.09,0.39)$ & $<0.001$ & $0.15(0.05,0.44)$ & 0.001 \\
\hline CPITN 2-3 & Slebioda 2011 & $4.10(2.13,7.89)$ & $<0.001$ & $4.20(2.07,8.52)$ & $<0.001$ & $3.83(1.45,10.11)$ & 0.007 \\
\hline CPITN 0-1 & Slebioda 2011 & $0.18(0.09,0.35)$ & $<0.001$ & $0.15(0.07,0.32)$ & $<0.001$ & $0.26(0.10,0.69)$ & 0.007 \\
\hline Dark indelible patches & Slebioda 2011 & $0.48(0.08,2.95)$ & 0.429 & $0.32(0.03,3.19)$ & 0.334 & $0.93(0.09,9.38)$ & 0.951 \\
\hline Deaquamative cheilitis & Slebioda 2011 & $1.11(0.18,6.82)$ & 0.911 & $1.00(0.14,7.31)$ & 1.000 & $1.42(0.12,16.34)$ & 0.780 \\
\hline Edentia & Slebioda 2011 & $6.93(0.37,130.94)$ & 0.196 & $9.54(0.50,180.64)$ & 0.133 & - & - \\
\hline Fissured tongue & Slebioda 2011 & $3.03(0.33,27.75)$ & 0.326 & $3.09(0.31,30.45)$ & 0.334 & $2.88(0.17,47.78)$ & 0.461 \\
\hline Fordyce's spots & Slebioda 2011 & $1.31(0.37,4.67)$ & 0.675 & $1.00(0.24,4.17)$ & 1.000 & $2.25(0.47,10.85)$ & 0.312 \\
\hline Geographic tongue & Slebioda 2011 & $1.89(0.36,10.03)$ & 0.455 & $2.06(0.37,11.63)$ & 0.413 & $1.42(0.12,16.34)$ & 0.780 \\
\hline Linea alba & Slebioda 2011 & $0.19(0.09,0.40)$ & $<0.001$ & $0.20(0.09,0.45)$ & $<0.001$ & $0.16(0.04,0.59)$ & 0.006 \\
\hline Palatal erythema & Slebioda 2011 & $2.05(0.53,8.04)$ & 0.301 & $2.88(0.73,11.35)$ & 0.130 & $0.38(0.02,7.58)$ & 0.525 \\
\hline Palatal papillary hyperplasia & Slebioda 2011 & $2.24(0.09,55.76)$ & 0.623 & $3.04(0.12,75.99)$ & 0.498 & - & - \\
\hline Recurrent aphthous stomatitis & Slebioda 2011 & $4.24(1.64,10.93)$ & 0.003 & $3.97(1.48,10.68)$ & 0.006 & $5.02(1.53,16.43)$ & 0.008 \\
\hline Recurrent labial herpes & Slebioda 2011 & $0.36(0.06,1.99)$ & 0.240 & $0.49(0.09,2.74)$ & 0.413 & $0.29(0.02,5.58)$ & 0.412 \\
\hline Rhomboid glossitis & Slebioda 2011 & $6.93(0.37,130.94)$ & 0.196 & $9.54(0.50,180.64)$ & 0.133 & - & - \\
\hline Sound teeth & Slebioda 2011 & $0.31(0.16,0.59)$ & $<0.001$ & $0.27(0.14,0.55)$ & $<0.001$ & $0.42(0.17,1.08)$ & 0.071 \\
\hline Swollen buccal mucosa & Slebioda 2011 & $7.98(2.30,27.66)$ & 0.001 & $10.24(2.90,36.16)$ & $<0.001$ & $3.05(0.57,16.20)$ & 0.192 \\
\hline Teeth with radicular or coronal decay & Slebioda 2011 & $3.27(1.71,6.27)$ & $<0.001$ & $3.69(1.83,7.43)$ & $<0.001$ & $2.36(0.93,6.01)$ & 0.071 \\
\hline White coated tongue & Slebioda 2011 & $1.62(0.87,3.03)$ & 0.132 & $1.50(0.77,2.95)$ & 0.234 & $2.01(0.77,5.25)$ & 0.156 \\
\hline White indelible patches and plates & Slebioda 2011 & $3.83(0.44,33.57)$ & 0.225 & $1.00(0.06,16.31)$ & 1.000 & $13.14(1.39,124.09)$ & 0.025 \\
\hline Buccal swelling & Zervou 2004 & $4.83(0.19,122.53)$ & 0.340 & $9.83(0.38,254.52)$ & 0.169 & - & - \\
\hline Buccal trauma & Zervou 2004 & $41.98(2.34,754.61)$ & 0.011 & $65.00(3.37,1253.85)$ & 0.006 & $26.60(1.29,549.44)$ & 0.034 \\
\hline Cobblestoning & Zervou 2004 & $12.09(0.60,242.89)$ & 0.103 & $26.60(1.29,549.44)$ & 0.034 & - & - \\
\hline Erythema migrans & Zervou 2004 & $4.83(0.19,122.53)$ & 0.340 & $9.83(0.38,254.52)$ & 0.169 & - & - \\
\hline
\end{tabular}




\begin{tabular}{|c|c|c|c|c|c|c|c|}
\hline Fissures elsewhere & Zervou 2004 & $12.09(0.60,242.89)$ & 0.103 & $26.60(1.29,549.44)$ & 0.034 & - & - \\
\hline Fissures lip & Zervou 2004 & $0.33(0.10,1.11)$ & 0.073 & $0.33(0.07,1.64)$ & 0.175 & $0.33(0.07,1.64)$ & 0.175 \\
\hline Fissures medline & Zervou 2004 & $0.14(0.03,0.66)$ & 0.013 & $0.30(0.06,1.49)$ & 0.140 & $0.06(0.00,1.10)$ & 0.058 \\
\hline Gingival bleeding & Zervou 2004 & $16.13(0.84,311.36)$ & 0.066 & $37.17(1.87,740.64)$ & 0.018 & - & - \\
\hline Gingivitis & Zervou 2004 & $16.13(0.84,311.36)$ & 0.066 & $26.60(1.29,549.44)$ & 0.034 & $9.83(0.38,254.52)$ & 0.169 \\
\hline Leukoplakia & Zervou 2004 & $4.83(0.19,122.53)$ & 0.340 & $9.83(0.38,254.52)$ & 0.169 & - & - \\
\hline Lip swelling & Zervou 2004 & $16.13(0.84,311.36)$ & 0.066 & $26.60(1.29,549.44)$ & 0.034 & $9.83(0.38,254.52)$ & 0.169 \\
\hline Lymphadenopathy & Zervou 2004 & $48.66(2.72,870.23)$ & 0.008 & $83.82(4.37,1608.44)$ & 0.003 & $26.60(1.29,549.44)$ & 0.034 \\
\hline Polypoid tags & Zervou 2004 & $12.09(0.60,242.89)$ & 0.103 & $26.60(1.29,549.44)$ & 0.034 & - & - \\
\hline Salivary gland involvement & Zervou 2004 & $16.13(0.84,311.36)$ & 0.066 & - & - & $37.17(1.87,740.64)$ & 0.018 \\
\hline
\end{tabular}

IBD, inflammatory bowel disease; CD, Crohn's disease; UC, ulcerative colitis; MD, mean difference; Cl, confidence interval; SG, subgroup; IL, interleukin; IFN, interferon; PI, plaque index; CAL, clinical attachment level; GCF; gingival crevicular fluid; PPD, pocket probing depth; P.a., Peptostreptococcus anaerobius; Pa.m., Parvimonas micra; Pr.m., Prevotella melaninogenica; Sta.a., Staphylococcus aureus; Str.a., Streptococcus anginosus; Str.mi., Streptococcus mitis; Str.mu., Streptococcus mutans; Tr.d., Treponema denticola; BOP, bleeding on probing; DMFT, decayed missing filled teeth; B.u., Bacteroides ureolyticus; C.g., Campylobacter gracilis; P.m., P. melaninogenica; S.ang., S. anginosus; S.aur., S. aureus; S.int., S. intermedius; S.mit., S. mitis; S.mut., S. mutans; TNF, tumor necrosis factor; DMFS, decayed missing filled tooth surfaces; GI, gingival index; GR, gingival recession; VPI, visible plaque index; OR, odds ratio; CPITN, community periodontal index treatment need. 
Appendix S5. Forest plot with the meta-analyses regarding the number of teeth. IBD, inflammatory bowel disease; $\mathrm{n}$, sample; SD, standard deviation; MD, mean difference; $\mathrm{Cl}$, confidence interval; Prl, predictive interval; $\mathrm{CD}$, Crohn's disease; UC, ulcerative colitis.

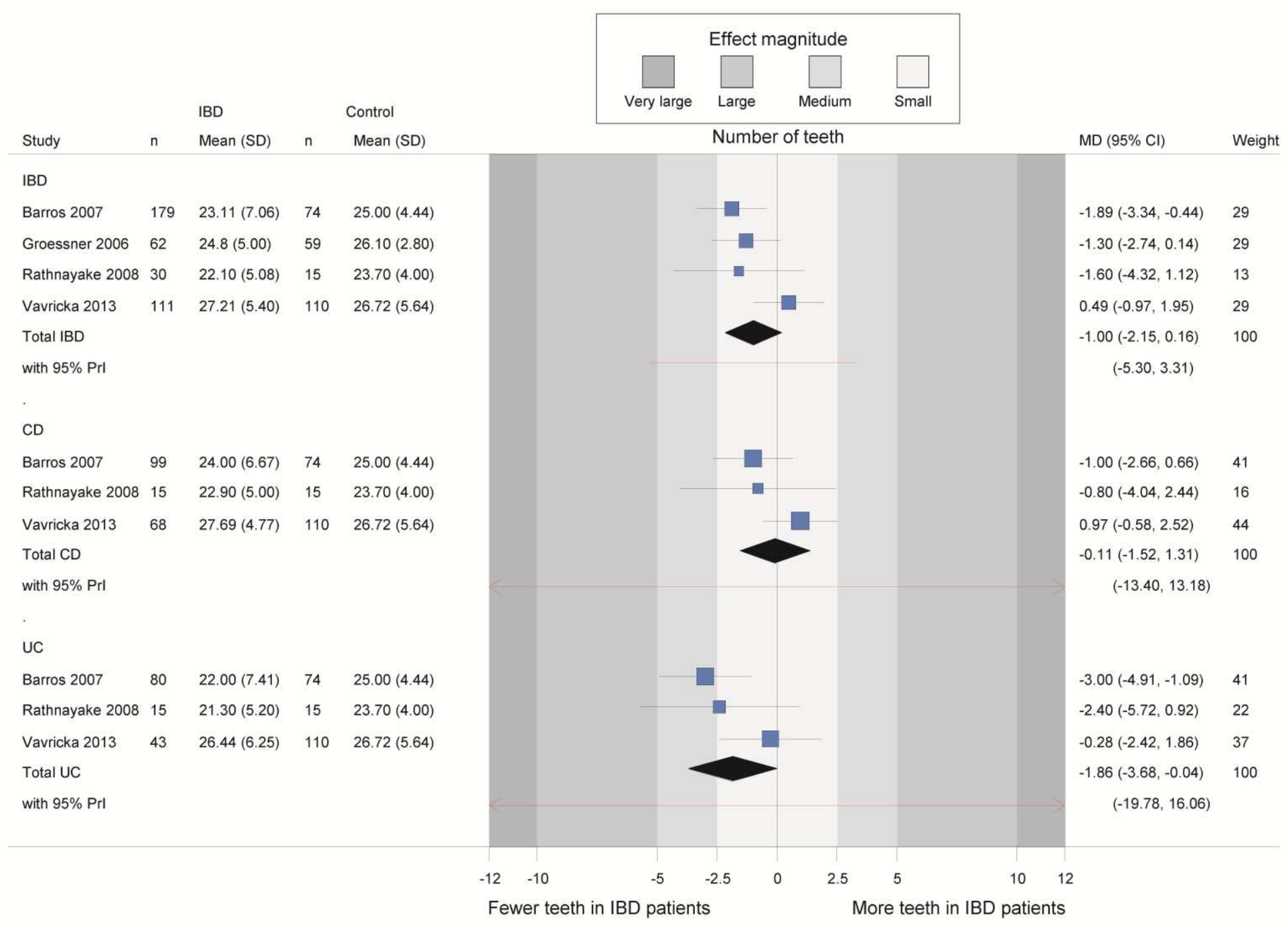


Appendix S6. Forest plot with the meta-analyses regarding the DMFT index. DMFT, decayed missing filled teeth; IBD, inflammatory bowel disease; $n$, sample; SD, standard deviation; MD, mean difference; Cl, confidence interval; Prl, predictive interval; CD, Crohn's disease; UC, ulcerative colitis.

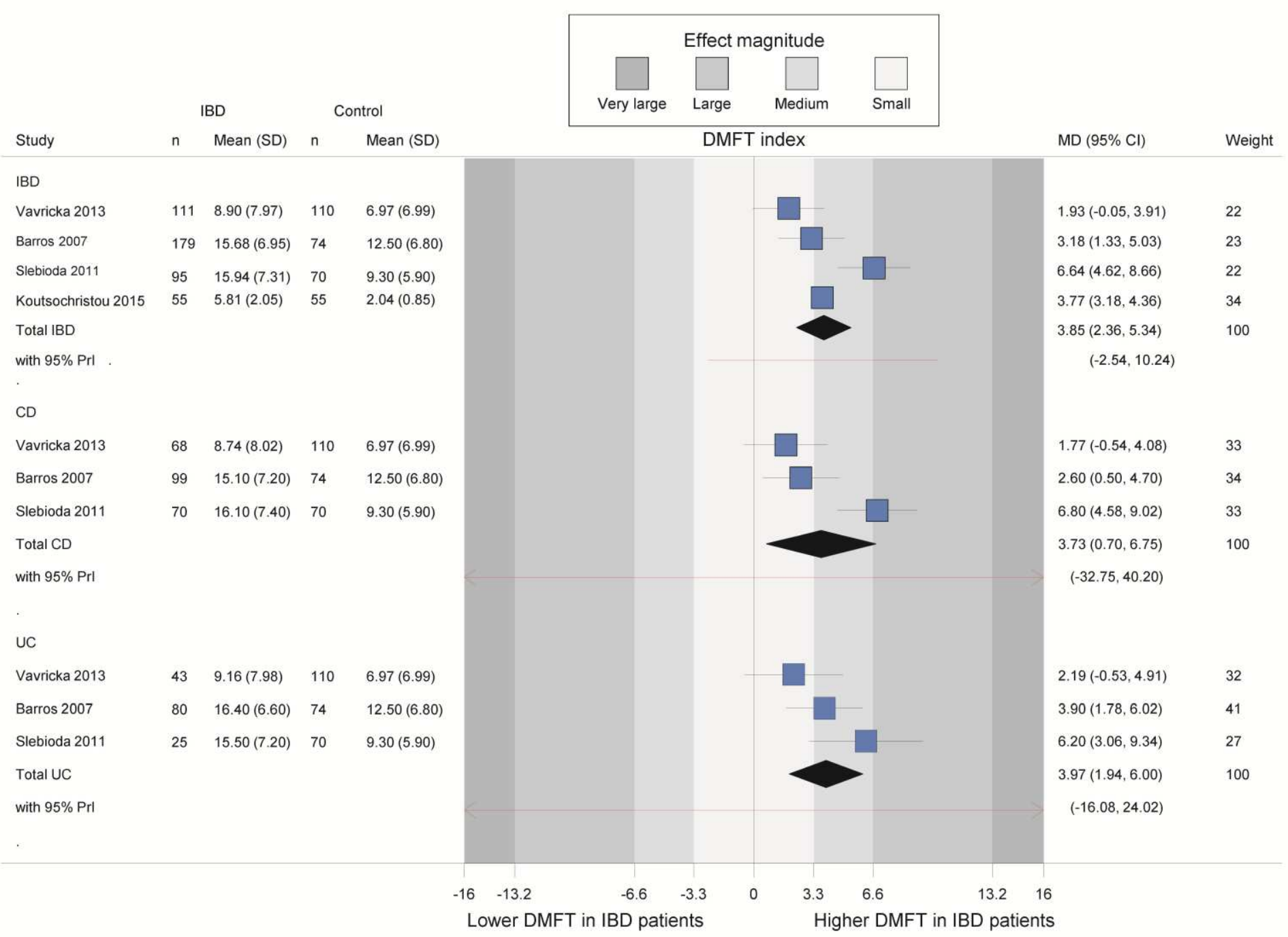


Appendix S7. Results of Meta-Analyses Comparing Patients With Crohn's Disease Compared to Healthy Patients

\begin{tabular}{|c|c|c|c|c|c|c|}
\hline & & \multicolumn{3}{|c|}{ Effect size } & \multicolumn{2}{|c|}{ Heterogeneity } \\
\hline Outcome & Studies & MD or OR $(95 \% \mathrm{Cl})$ & $p$ & $95 \% \mathrm{Prl}$ & $\mathrm{I}^{2}(95 \% \mathrm{Cl})$ & $\mathrm{T}^{2}$ \\
\hline \multicolumn{7}{|l|}{ Primary outcome } \\
\hline Periodontitis & 4 & OR: $3.96(2.39,6.54)$ & $<0.001$ & $1.31,11.92$ & $0(0,68)$ & 0 \\
\hline \multicolumn{7}{|l|}{ Secondary outcomes } \\
\hline Number of teeth & 3 & MD: $-0.11(-1.52,1.31)$ & 0.881 & $-13.40,13.18$ & $36(0,82)$ & 0.572 \\
\hline DMFT index & $3^{*}$ & MD: $3.73(0.70,6.75)$ & 0.016 & $-32.75,40.20$ & $82(0,92)$ & 5.863 \\
\hline Plaque index & c & & & & & \\
\hline Bleeding on probing & a & & & & & \\
\hline Clinical attachment loss & $\mathrm{b}$ & & & & & \\
\hline Clinical attachment loss $>3 \mathrm{~mm}$ & 2 & MD: $2.42(-2.49,7.33)$ & 0.333 & NA & 0 (NA) & 0 \\
\hline Pocket probing depth & $3^{*}$ & MD: $0.56(-0.01,1.13)$ & 0.054 & $-6.67,7.79$ & $95(90,97)$ & 0.239 \\
\hline Pocket probing depth $>3 \mathrm{~mm}$ & 2 & MD: $4.82(0.65,8.99)$ & 0.024 & NA & 0 (NA) & 0 \\
\hline Pocket probing depth $>4 \mathrm{~mm}$ & $3^{*}$ & MD: -3.49 (-9.41,2.43) & 0.248 & $-73.87,66.89$ & $90(67,95)$ & 21.550 \\
\hline Oral lesions & $2^{*}$ & OR: $5.17(0.53,50.58)$ & 0.158 & NA & $96(\mathrm{NA})$ & 2.590 \\
\hline Angular cheilitis & 2 & OR: $10.04(0.85,118.26)$ & 0.067 & NA & $56(\mathrm{NA})$ & 1.877 \\
\hline Aphthous lesions & 2 & OR: $1.99(0.56,7.08)$ & 0.289 & NA & $74(\mathrm{NA})$ & 0.626 \\
\hline Hairy tongue & 2 & OR: $9.44(1.07,83.01)$ & 0.043 & NA & $0(\mathrm{NA})$ & 0 \\
\hline Ulceration & 2 & OR: $11.16(1.30,95.55)$ & 0.028 & NA & $0(\mathrm{NA})$ & 0 \\
\hline
\end{tabular}

$\mathrm{MD}$, mean difference; OR, odds ratio; $\mathrm{Cl}$, confidence interval; Prl, predictive interval; NA, not applicable; DMFT, decayed missing filling teeth. *extreme heterogeneity, but agrees on direction; effect magnitude might be misestimated.

${ }^{a}$ Meta-analysis omitted; three studies with extreme heterogeneity; studies evenly distributed $\left(\mathrm{MD}=1.65 ; 95 \% \mathrm{Cl}=-7.06,10.37 ; \mathrm{p}=0.710 ; \mathrm{I}^{2}=77\right)$

b Meta-analysis omitted; three studies with extreme heterogeneity; studies evenly distributed (MD=0.013;95\% Cl=-0.42,0.45; $\mathrm{p}=0.954 ; \mathrm{I}^{2}=78$ )

c Meta-analysis omitted; three studies with extreme heterogeneity; studies evenly distributed (Standardized Mean Difference=-0.05; $95 \% \mathrm{Cl}=-0.77,0.67$;

$\left.\mathrm{p}=0.891 ; \mathrm{I}^{2}=90\right)$ 
Appendix S8. Results of Meta-Analyses Comparing Patients With Ulcerative Colitis Compared to Healthy Patients

\begin{tabular}{|c|c|c|c|c|c|c|}
\hline & & \multicolumn{3}{|c|}{ Effect size } & \multicolumn{2}{|c|}{ Heterogeneity } \\
\hline Outcome & Studies & MD or OR $(95 \% \mathrm{Cl})$ & $p$ & $95 \% \mathrm{Prl}$ & $\mathrm{I}^{2}(95 \% \mathrm{Cl})$ & $\mathrm{T}^{2}$ \\
\hline \multicolumn{7}{|l|}{ Primary outcome } \\
\hline Periodontitis & 3 & OR: $5.11(2.92,8.92)$ & $<0.001$ & $0.14,189.85$ & $0(0,73)$ & 0 \\
\hline \multicolumn{7}{|l|}{ Secondary outcomes } \\
\hline Number of teeth & 3 & MD: -1.86 (-3.68,-0.04) & 0.045 & $-19.78,16.06$ & $44(0,83)$ & 1.128 \\
\hline DMFT index & 3 & MD: $3.97(1.94,6.00)$ & $<0.001$ & $-16.08,24.04$ & $44(0,83)$ & 1.420 \\
\hline Plaque index & $2^{\mathrm{a},{ }^{*}}$ & MD: $0.50(-0.42,1.41)$ & 0.289 & NA & 95 (NA) & 0.415 \\
\hline Bleeding on probing & 3 & MD: $1.42(-5.01,7.86)$ & 0.665 & $-69.58,72.43$ & $67(0,88)$ & 20.444 \\
\hline Clinical attachment loss & 3 & MD: $0.40(-0.02,0.828)$ & 0.061 & $-4.40,5.21$ & $74(0,90)$ & 0.096 \\
\hline Clinical attachment loss $>3 \mathrm{~mm}$ & 2 & MD: $13.68(7.29,20.08)$ & $<0.001$ & NA & 7 (NA) & 1.515 \\
\hline Pocket probing depth & $3^{*}$ & MD: $0.66(0.27,1.05)$ & 0.001 & $-4.22,5.54$ & $96(91,97)$ & 0.108 \\
\hline Pocket probing depth $>3 \mathrm{~mm}$ & 2 & MD: $0.55(-10.43,11.54)$ & 0.921 & NA & $64(\mathrm{NA})$ & 44.715 \\
\hline Pocket probing depth $>4 \mathrm{~mm}$ & b & & & & & \\
\hline Oral lesions & $2^{*}$ & OR: $6.27(1.53,25.70)$ & 0.011 & NA & $87(\mathrm{NA})$ & 0.906 \\
\hline Angular cheilitis & 2 & OR: $4.54(0.07,288.50)$ & 0.476 & NA & $73(\mathrm{NA})$ & 6.588 \\
\hline Aphthous lesions & 3 & OR: $3.38(1.75,6.53)$ & $<0.001$ & $0.05,242.46$ & $0(0,73)$ & 0 \\
\hline Hairy tongue & 2 & OR: $9.21(0.93,91.25)$ & 0.058 & NA & $0(\mathrm{NA})$ & 0 \\
\hline Ulceration & 2 & OR: $9.21(0.93,91.25)$ & 0.058 & NA & $0(\mathrm{NA})$ & 0 \\
\hline
\end{tabular}

$\mathrm{MD}$, mean difference; OR, odds ratio; $\mathrm{Cl}$, confidence interval; Prl, predictive interval; NA, not applicable; DMFT, decayed missing filling teeth

*extreme heterogeneity, but agrees on direction; effect magnitude might be misestimated.

a Omitted study of Rathnayake 2008 to achieve homogeneity (Standardized Mean Difference (SMD) $=0.29 ; 95 \% \mathrm{Cl}=-0.47,1.04 ; \mathrm{p}=0.460 ; \mathrm{I}^{2}=91$ ); used SMD to combine two plaque indeces

b Meta-analysis omitted; three studies with extreme heterogeneity; studies evenly distributed $\left(\mathrm{MD}=-1.73 ; 95 \% \mathrm{Cl}=-5.78,2.33 ; \mathrm{p}=0.404 ; \mathrm{I}^{2}=75\right)$ 
Appendix S9. Additional details about the Summary of Findings table (Table 4).

\begin{tabular}{|c|c|c|c|c|c|c|c|c|}
\hline Outcome & $\begin{array}{l}\text { Risk of } \\
\text { Bias }\end{array}$ & Inconsistency & Indirectness & Imprecision & Publication bias & Large Effect & $\begin{array}{c}\text { Dose } \\
\text { Response }\end{array}$ & Residual Confounding \\
\hline $\begin{array}{l}\text { Periodontitis } \\
\text { IBD }\end{array}$ & \begin{tabular}{|l|} 
No \\
serious \\
limitations
\end{tabular} & $\begin{array}{l}\text { Little to no } \\
\text { heterogeneity; no } \\
\text { apparent reason to } \\
\text { downgrade. }\end{array}$ & $\begin{array}{l}\text { Directly relevant; } \\
\text { no apparent } \\
\text { reason to } \\
\text { downgrade. }\end{array}$ & $\begin{array}{l}\text { Adequate sample; no } \\
\text { apparent reason to } \\
\text { downgrade. }\end{array}$ & $\begin{array}{l}\text { Small number of studies } \\
\text { precluded formal assessment } \\
\text { of reporting bias; no apparent } \\
\text { reason to downgrade. }\end{array}$ & $\begin{array}{l}\text { Rated up by two levels for very } \\
\text { large effect included in the } \\
\text { confidence and predictive } \\
\text { interval. }\end{array}$ & $\begin{array}{l}\text { No reason } \\
\text { to rate up }\end{array}$ & $\begin{array}{l}\text { Rated up by one; estimates } \\
\text { adjusted for confounders } \\
\text { confirmed results agree with } \\
\text { original analyses. }\end{array}$ \\
\hline $\begin{array}{l}\text { Periodontitis } \\
\text { CD }\end{array}$ & $\begin{array}{l}\text { No } \\
\text { serious } \\
\text { limitations }\end{array}$ & $\begin{array}{l}\text { Little to no } \\
\text { heterogeneity; no } \\
\text { apparent reason to } \\
\text { downgrade. }\end{array}$ & $\begin{array}{l}\text { Directly relevant; } \\
\text { no apparent } \\
\text { reason to } \\
\text { downgrade. } \\
\end{array}$ & $\begin{array}{l}\text { Adequate sample; no } \\
\text { apparent reason to } \\
\text { downgrade. }\end{array}$ & $\begin{array}{l}\text { Small number of studies } \\
\text { precluded formal assessment } \\
\text { of reporting bias; no apparent } \\
\text { reason to downgrade. }\end{array}$ & $\begin{array}{l}\text { Rated up by one level for large } \\
\text { effect included in the } \\
\text { confidence and predictive } \\
\text { interval. }\end{array}$ & $\begin{array}{l}\text { No reason } \\
\text { to rate up }\end{array}$ & $\begin{array}{l}\text { Rated up by one; estimates } \\
\text { adjusted for confounders } \\
\text { confirmed results agree with } \\
\text { original analyses. }\end{array}$ \\
\hline $\begin{array}{l}\text { Periodontitis } \\
\text { UC }\end{array}$ & $\begin{array}{l}\text { No } \\
\text { serious } \\
\text { limitations }\end{array}$ & $\begin{array}{l}\text { Little to no } \\
\text { heterogeneity; no } \\
\text { apparent reason to } \\
\text { downgrade. }\end{array}$ & $\begin{array}{l}\text { Directly relevant; } \\
\text { no apparent } \\
\text { reason to } \\
\text { downgrade. }\end{array}$ & $\begin{array}{l}\text { Adequate sample; no } \\
\text { apparent reason to } \\
\text { downgrade. }\end{array}$ & $\begin{array}{l}\text { Small number of studies } \\
\text { precluded formal assessment } \\
\text { of reporting bias; no apparent } \\
\text { reason to downgrade. }\end{array}$ & $\begin{array}{l}\text { Rated up by two levels for very } \\
\text { large effect included in the } \\
\text { confidence and predictive } \\
\text { interval. }\end{array}$ & $\begin{array}{l}\text { No reason } \\
\text { to rate up }\end{array}$ & $\begin{array}{l}\text { Rated up by one; estimates } \\
\text { adjusted for confounders } \\
\text { confirmed results agree with } \\
\text { original analyses. }\end{array}$ \\
\hline No of teeth IBD & $\begin{array}{l}\text { No } \\
\text { serious } \\
\text { limitations }\end{array}$ & $\begin{array}{l}\text { Little to no } \\
\text { heterogeneity; no } \\
\text { apparent reason to } \\
\text { downgrade. }\end{array}$ & $\begin{array}{l}\text { Directly relevant; } \\
\text { no apparent } \\
\text { reason to } \\
\text { downgrade. }\end{array}$ & $\begin{array}{l}\text { Adequate sample; no } \\
\text { apparent reason to } \\
\text { downgrade. }\end{array}$ & $\begin{array}{l}\text { Small number of studies } \\
\text { precluded formal assessment } \\
\text { of reporting bias; no apparent } \\
\text { reason to downgrade. }\end{array}$ & No reason to rate up & $\begin{array}{l}\text { No reason } \\
\text { to rate up }\end{array}$ & No reason to rate up \\
\hline No of teeth CD & $\begin{array}{l}\text { No } \\
\text { serious } \\
\text { limitations }\end{array}$ & $\begin{array}{l}\text { Little to no } \\
\text { heterogeneity; no } \\
\text { apparent reason to } \\
\text { downgrade. }\end{array}$ & $\begin{array}{l}\text { Directly relevant; } \\
\text { no apparent } \\
\text { reason to } \\
\text { downgrade. }\end{array}$ & $\begin{array}{l}\text { Adequate sample; no } \\
\text { apparent reason to } \\
\text { downgrade. }\end{array}$ & $\begin{array}{l}\text { Small number of studies } \\
\text { precluded formal assessment } \\
\text { of reporting bias; no apparent } \\
\text { reason to downgrade. }\end{array}$ & No reason to rate up & $\begin{array}{l}\text { No reason } \\
\text { to rate up }\end{array}$ & No reason to rate up \\
\hline No of teeth UC & $\begin{array}{l}\text { No } \\
\text { serious } \\
\text { limitations }\end{array}$ & $\begin{array}{l}\text { Little to no } \\
\text { heterogeneity; no } \\
\text { apparent reason to } \\
\text { downgrade. }\end{array}$ & $\begin{array}{l}\text { Directly relevant; } \\
\text { no apparent } \\
\text { reason to } \\
\text { downgrade. }\end{array}$ & $\begin{array}{l}\text { Adequate sample; no } \\
\text { apparent reason to } \\
\text { downgrade. }\end{array}$ & $\begin{array}{l}\text { Small number of studies } \\
\text { precluded formal assessment } \\
\text { of reporting bias; no apparent } \\
\text { reason to downgrade. }\end{array}$ & No reason to rate up & $\begin{array}{l}\text { No reason } \\
\text { to rate up }\end{array}$ & No reason to rate up \\
\hline DMFT IBD & $\begin{array}{l}\text { No } \\
\text { serious } \\
\text { limitations }\end{array}$ & $\begin{array}{l}\text { Extreme } \\
\text { heterogeneity; } \\
\text { however effects agree } \\
\text { in direction and } \\
\text { confidence regarding } \\
\text { decision is unaffected; } \\
\text { no apparent reason to } \\
\text { downgrade. }\end{array}$ & $\begin{array}{l}\text { Directly relevant; } \\
\text { no apparent } \\
\text { reason to } \\
\text { downgrade. }\end{array}$ & $\begin{array}{l}\text { Adequate sample; no } \\
\text { apparent reason to } \\
\text { downgrade. }\end{array}$ & $\begin{array}{l}\text { Small number of studies } \\
\text { precluded formal assessment } \\
\text { of reporting bias; no apparent } \\
\text { reason to downgrade. }\end{array}$ & No reason to rate up & $\begin{array}{l}\text { No reason } \\
\text { to rate up }\end{array}$ & No reason to rate up \\
\hline DMFT CD & $\begin{array}{l}\text { No } \\
\text { serious } \\
\text { limitations }\end{array}$ & $\begin{array}{l}\text { Extreme } \\
\text { heterogeneity; } \\
\text { however effects agree } \\
\text { in direction and } \\
\text { confidence regarding } \\
\text { decision is unaffected; } \\
\text { no apparent reason to } \\
\text { downgrade. }\end{array}$ & $\begin{array}{l}\text { Directly relevant; } \\
\text { no apparent } \\
\text { reason to } \\
\text { downgrade. }\end{array}$ & $\begin{array}{l}\text { Adequate sample; no } \\
\text { apparent reason to } \\
\text { downgrade. }\end{array}$ & $\begin{array}{l}\text { Small number of studies } \\
\text { precluded formal assessment } \\
\text { of reporting bias; no apparent } \\
\text { reason to downgrade. }\end{array}$ & No reason to rate up & $\begin{array}{l}\text { No reason } \\
\text { to rate up }\end{array}$ & No reason to rate up \\
\hline DMFT UC & $\begin{array}{l}\text { No } \\
\text { serious } \\
\text { limitations }\end{array}$ & $\begin{array}{l}\text { Little to no } \\
\text { heterogeneity; no } \\
\text { apparent reason to } \\
\text { downgrade. }\end{array}$ & $\begin{array}{l}\text { Directly relevant; } \\
\text { no apparent } \\
\text { reason to } \\
\text { downgrade. }\end{array}$ & $\begin{array}{l}\text { Adequate sample; no } \\
\text { apparent reason to } \\
\text { downgrade. }\end{array}$ & $\begin{array}{l}\text { Small number of studies } \\
\text { precluded formal assessment } \\
\text { of reporting bias; no apparent } \\
\text { reason to downgrade. }\end{array}$ & No reason to rate up & $\begin{array}{l}\text { No reason } \\
\text { to rate up }\end{array}$ & No reason to rate up \\
\hline
\end{tabular}

IBD, inflammatory bowel disease; CD, Crohn's disease; UC, ulcerative colitis; DMFT, decayed-missing-filled-teeth index. 
Appendix S10. Exploratory comparison of differences between UC and CD patients (UC minus CD).

\begin{tabular}{|c|c|c|c|c|c|c|}
\hline \multirow[b]{2}{*}{ Outcome } & \multirow[b]{2}{*}{ Studies } & \multicolumn{3}{|l|}{ Effect size } & \multicolumn{2}{|c|}{ Heterogeneity } \\
\hline & & MD or OR $(95 \% \mathrm{Cl})$ & $\mathbf{P}$ & $95 \% \mathrm{Prl}$ & $\mathrm{I}^{2}(95 \% \mathrm{Cl})$ & $\mathrm{T}^{2}$ \\
\hline \multicolumn{7}{|l|}{ Primary outcome } \\
\hline Periodontitis & 2 & OR: $0.86(0.11,6.58)$ & 0.884 & NA & 44 (NA) & 1.129 \\
\hline \multicolumn{7}{|l|}{ Secondary outcomes } \\
\hline Number of teeth & 4 & MD: -1.67 (-2.97,-0.34) & 0.013 & $-10.68,7.41$ & $0(0,73)$ & 0 \\
\hline DMFT index & 3 & MD: $0.69(-0.81,2.20)$ & 0.365 & $-9.05,10.44$ & $0(0,73)$ & 0 \\
\hline Plaque index & 3 & SMD: $0.33(0.13,0.54)$ & 0.002 & $-1.02,1.69$ & $0(0,73)$ & 0 \\
\hline Bleeding on probing & 3 & MD: $0.41(-2.91,3.72)$ & 0.811 & $-21.09,21.90$ & $0(0,73)$ & 0 \\
\hline Clinical attachment loss & 3 & MD: $0.40(0.20,0.59)$ & $<0.001$ & $-0.89,1.68$ & $0(0,73)$ & 0 \\
\hline Clinical attachment loss $>3 \mathrm{~mm}$ & 2 & MD: $10.18(4.10,16.25)$ & $<0.001$ & NA & $0(0,73)$ & 0 \\
\hline Pocket probing depth & 3 & MD: $0.12(-0.03,0.28)$ & 0.124 & $-1.33,1.58$ & $33(0,81)$ & 0.001 \\
\hline Pocket probing depth $>3 \mathrm{~mm}$ & * & & & & & \\
\hline Pocket probing depth $>4 \mathrm{~mm}$ & 3 & MD: $1.33(-0.80,3.45)$ & 0.220 & $-21.64,24.30$ & $69(0,89)$ & 2.092 \\
\hline Angular cheilitis & 2 & OR: $0.52(0.13,2.07)$ & 0.354 & NA & 0 (NA) & 0 \\
\hline Aphthous lesions & 3 & OR: $1.86(0.97,3.58)$ & 0.063 & $0.03,129.72$ & $0(0,73)$ & 0 \\
\hline Hairy tongue & 2 & OR: $0.82(0.14,4.76)$ & 0.829 & NA & 0 (NA) & 0 \\
\hline Ulceration & 2 & OR: $0.68(0.12,3.71)$ & 0.653 & NA & 0 (NA) & 0 \\
\hline
\end{tabular}

UC, ulcerative colitis; CD, Crohn's disease; MD, mean difference; OR, odds ratio; CI, confidence interval; Prl, predictive interval; NA, not applicable; DMFT, decayed missing filling teeth.

* Meta-analysis omitted; two studies with extreme heterogeneity; studies evenly distributed $\left(\mathrm{MD}=-0.59 ; 95 \% \mathrm{Cl}=-18.56,17.37 ; \mathrm{P}=0.949 ; \mathrm{I}^{2}=88\right)$ 
Appendix S11. Results of random-effects meta-regression according to patient characteristics.

\begin{tabular}{|c|c|c|c|c|c|c|c|c|c|c|c|c|c|c|c|}
\hline & \multicolumn{3}{|c|}{ Age } & \multicolumn{3}{|c|}{ Sex } & \multicolumn{3}{|c|}{ Smokers } & \multicolumn{3}{|c|}{ Active disease } & \multicolumn{3}{|c|}{ Medication use } \\
\hline & $\mathrm{n}$ & Coefficient $(95 \% \mathrm{Cl})$ & $P$ & $\mathrm{n}$ & Coefficient $(95 \% \mathrm{Cl})$ & $\mathrm{P}$ & $\mathrm{n}$ & Coefficient $(95 \% \mathrm{Cl})$ & $\mathrm{P}$ & $\mathrm{n}$ & Coefficient $(95 \% \mathrm{Cl})$ & $P$ & $\mathrm{n}$ & Coefficient $(95 \% \mathrm{Cl})$ & $\mathrm{P}$ \\
\hline Periodontitis & 3 & $-0.66(-18.59,17.26)$ & 0.720 & 3 & $0.43(-6.93,7.78)$ & 0.594 & 3 & $1.59(-19.73,22.91)$ & 0.517 & - & 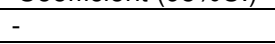 & - & 3 & $-0.54(-32.66,31.58)$ & 0.867 \\
\hline No of teeth & 4 & $0.01(-2.61,2.62)$ & 0.992 & 4 & $2.66(-2.41,7.74)$ & 0.152 & 4 & $6.15(-9.18,21.47)$ & 0.226 & 3 & $3.50(-93.22,100.22)$ & 0.726 & 4 & $-7.94(-23.40,7.52)$ & 0.158 \\
\hline DMFT & 3 & $-1.14(-6.34,4.06)$ & 0.219 & 3 & $0.88(-68.93,70.70)$ & 0.898 & - & - & & - & - & - & - & & \\
\hline
\end{tabular}

$\mathrm{n}$, sample; $\mathrm{Cl}$, confidence interval; DMFT, decayed missing filled teeth index. 
Appendix S12. Sensitivity analysis according to the periodontitis definition.

\begin{tabular}{|c|c|c|c|c|c|c|}
\hline Study & \multirow{2}{*}{\multicolumn{6}{|c|}{$\begin{array}{ll} & \text { Definition of periodontitis }\end{array}$}} \\
\hline Barros 2007 & \multicolumn{2}{|c|}{ "Periodontitis was defined as the presence of at least four sites in different teeth with CAL $\geq 3 \mathrm{~mm}$." } & & & & \\
\hline Vavricka 2013 & \multicolumn{6}{|c|}{ "Crude differences regarding risk factors for periodontitis, assessed by the oral health markers LA-PPD for periodontitis..." } \\
\hline Habasneh 2012 & \multicolumn{6}{|c|}{$\begin{array}{l}\text { "Periodontitis was defined as presence of four or more teeth with one site or more having a probing pocket depth } \geq 4 \mathrm{~mm} \text { and clinical } \\
\text { attachment level } \geq 3 \mathrm{~mm} \text {." }\end{array}$} \\
\hline Zervou 2004 & \multicolumn{6}{|l|}{ No definition given. } \\
\hline & \multicolumn{2}{|c|}{ IBD } & \multicolumn{2}{|c|}{ CD } & UC & \\
\hline & OR (95\% Cl) & $\mathbf{P}$ & OR (95\% Cl) & $\mathbf{P}$ & OR (95\% Cl) & $\mathbf{P}$ \\
\hline Original & $4.55(3.00,6.91)$ & $<0.001$ & $3.96(2.39,6.54)$ & $<0.001$ & $5.11(2.92,8.92)$ & $<0.001$ \\
\hline Sensitivity analysis & $6.07(2.96)$ & $<0.001$ & $4.82(2.88,8.08)$ & $<0.001$ & $3.70(1.89,7.24)$ & $<0.001$ \\
\hline
\end{tabular}

CAL, clinical attachment loss; LA-PPD, loss of attachment at sites with maximal periodontal pocket depth; IBD, inflammatory bowel disease; CD, Crohn's disease; UC, ulcerative colitis; OR, odds ratio. 
Appendix S13. Criteria used to diagnose Inflammatory Bowel Disease (IBD), Crohn's Disease (CD), and Ulcerative Colitis (UC).

\begin{tabular}{|c|c|c|}
\hline Nr. & Study & Diagnosis \\
\hline 1 & Barros 2007 & The diagnosis of CD or UC had been established previously by clinical, radiological, endoscopic and histological analyses. \\
\hline 2 & $\begin{array}{l}\text { Groessner-Schreiber } \\
2006\end{array}$ & $\begin{array}{l}\text { The diagnosis of either CD or UC was confirmed by previously established clinical, radiological and endoscopic criteria (type of lesions, } \\
\text { distribution*), and histological findings also had to be confirmative or compatible with this diagnosis. }\end{array}$ \\
\hline 3 & Habashneh 2012 & The diagnosis of IBD was established by clinical, radiological, endoscopic and histological criteria. \\
\hline 4 & Koutsochristou 2015 & The diagnosis of CD or UC was established according to the Porto criteria.34 \\
\hline 45 & Rathnayake 2008 & The diagnosis of either Crohn's disease (CD) or Ulcerative colitis (UC) was conformed by clinical, radiographic and endoscopic criteria. \\
\hline 56 & Slebioda 2011 & Not specified. \\
\hline 67 & Van Dyke 1986 & $\begin{array}{l}\text { IBD patients were defined as those exhibiting a combination of symptoms, including diarrhea, abdominal pain, bleeding, weight loss, } \\
\text { perianal disease, and arthritis. }\end{array}$ \\
\hline 78 & Vavricka 2013 & The diagnosis of CD or UC had been established previously by clinical, radiological, endoscopic, and histological analyses. \\
\hline 89 & Zervou 2007 & Not specified. \\
\hline
\end{tabular}


Appendix S14. Additional review data

Author Contributions

SNP designed the protocol/datasheets, registered the review, designed and performed the literature searches, extracted search results, performed study screening, performed study selection, extracted data from studies, performed study quality assessment, performed statistical analysis, graded the quality of evidence with the GRADE approach, wrote first draft of the manuscript, checked and approved all data prior to submission, submitted the paper, and is responsible for the accuracy of the results.

$\mathrm{MH}$ checked the protocol/datasheets, performed study selection, extracted data from studies, performed study quality assessment, aided in the interpretation of the analyses, contributed to manuscript writing, checked and approved all data prior to submission

AVBN aided in the interpretation of the analyses, contributed to manuscript writing, checked and approved all data prior to submission

AF aided in the interpretation of the analyses, contributed to manuscript writing, checked and approved all data prior to submission

JD conceived the experiment, checked the protocol/datasheets, resolved conflict during the duplicate procedures, aided in the interpretation of the analyses, contributed to manuscript writing, checked and approved all data prior to submission, and is the corresponding author.

Changes to the protocol after initiation

1. The primary outcome remained the one that was pre-specified; the secondary outcomes were decided upon post hoc evaluation of all identified outcomes (giving priority to outcomes reported by most studies). As such the title

2. The SMD was used for one meta-analysis instead of the MD that was originally planned, in order to combine two similar plaque indeces.

3. Many additional outcomes (periodontal progression and response to periodontal treatment), as well as analyses (subgroup analyses, reporting bias, sensitivity analyses) were either not possible or partly possible to conduct, due to the number and nature of the included studies.

4. According to bullet's 1 and 3 , the scope and title of the review was changed from a periodontitis-based one to a more general oral-health-related one, although as stated in bullet 1 , the primary outcome remained as pre-specified. 
5. After peer review, the literature searches were updated in January 2017 and the results from the new searches were integrated in the revised submission. 